\title{
Spin-dependent gravitational tail memory in $D=4$
}

\author{
Debodirna Ghosh $\odot^{1, *}$ and Biswajit Sahoo $\odot^{2, \dagger}$ \\ ${ }^{1}$ Chennai Mathematical Institute, Siruseri, Kelambakkam 603103, Chennai, India \\ ${ }^{2}$ Fields and Strings Laboratory, Institute of Physics, Ecole Polytechnique Federale de Lausanne (EPFL), \\ CH-1015 Lausanne, Switzerland
}

(Received 17 October 2021; accepted 9 January 2022; published 28 January 2022)

\begin{abstract}
We derive the leading spin-dependent gravitational tail memory, which appears at the second postMinkowskian order and behaves as $u^{-2}$ for large retarded time $u$. This result follows from the classical soft graviton theorem at order $\omega \ln \omega$ as a low-frequency expansion of the gravitational waveform with frequency $\omega$. First, we conjecture the gravitational waveform from the classical limit of the quantum soft graviton theorem up to sub-subleading order in a soft expansion, and then we derive it for a classical scattering process without any reference to the soft graviton theorem. We show that the final result of the gravitational waveform in the direct derivation completely agrees with the conjectured waveform.
\end{abstract}

DOI: 10.1103/PhysRevD.105.025024

\section{INTRODUCTION}

The observation of the permanent displacement between the mirrors of the gravitational wave detector relative to their initial distance after the passage of full gravitational radiation produced from an astrophysical scattering event is known as gravitational memory. Nonoscillatory sources, e.g., scattering of two unbounded compact objects in hyperbolic orbit, contribute to "linear memory," which can be read off from the matter energy-momentum tensor determined in terms of the trajectories of the scattered objects [1-3]. In companion with this, there will also be "nonlinear memory" due to gravitational radiation from the gravitational waves produced during the scattering process, which can be read off studying the gravitational energymomentum tensor [4-8]. The nonlinear memory effect is always present for any gravitational scattering process whether it is bounded or not. In the near future there is a hope of direct detection of gravitational memory in the upcoming gravitational wave detectors $[9,10]$.

In recent years, there has been a proposal of another kind of gravitational memory known as "tail memory," which describes how the mirrors of a gravitational wave detector behave at a large retarded time before reaching their permanent displaced positions [11-15]. The late and early time gravitational waveforms responsible for gravitational

\footnotetext{
debodirna@cmi.ac.in

tbiswajit.sahoo@epfl.ch
}

Published by the American Physical Society under the terms of the Creative Commons Attribution 4.0 International license. Further distribution of this work must maintain attribution to the author(s) and the published article's title, journal citation, and DOI. Funded by SCOAP . tail memories are first conjectured from the classical limit of the soft graviton theorem with some infrared regulator prescription [12,16,17], and then derived explicitly in the name of the classical soft graviton theorem $[13,14]$. It has also been shown that these late and early time behaviors of the gravitational waveform are related to the radiative mode of low frequency gravitational waveform via Fourier transformation in the frequency variable. In $[13,14]$, the setup considered for the scattering event is schematically described in Fig. 1. Consider $M$ number of objects are coming in from the asymptotic past with masses $\left\{m_{a}^{\prime}\right\}$, momenta $\left\{p_{a}^{\prime}\right\}$, and spins $\left\{\Sigma_{a}^{\prime}\right\}$ going through some unspecified interaction within region $\mathcal{R}$ of spacetime size $L$ and disperse to $N$ number of objects with masses $\left\{m_{a}\right\}$, momenta $\left\{p_{a}\right\}$, and spins $\left\{\Sigma_{a}\right\}$. These incoming and outgoing objects can also be massless radiations. We choose the origin of the coordinate system (i.e., scattering center) well inside this region $\mathcal{R}$, and we place the gravitational wave detector at a distance $R$ from this origin, along the direction $\hat{n}$. Now we define the deviation of the metric from the Minkowski background as

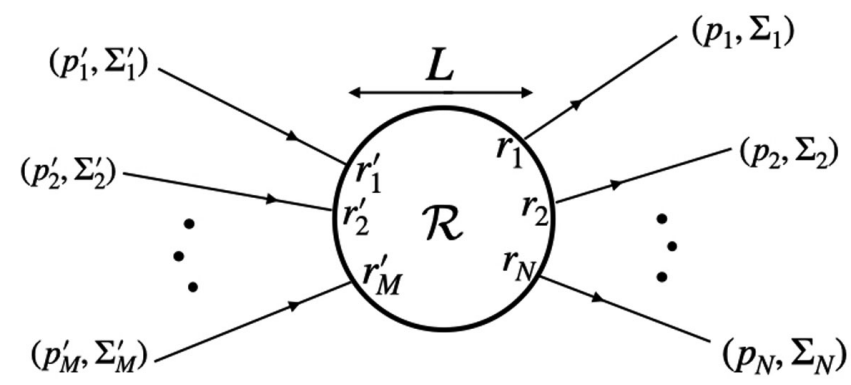

FIG. 1. The setup of the gravitational scattering event. 


\begin{tabular}{|c|c|c|c|c|c|c|}
\hline $\begin{array}{c}\text { o-loop (tree) } \\
\mathcal{O}(G)\end{array}$ & $\begin{array}{l}\omega^{-1} \\
\theta(u)\end{array}$ & $\begin{array}{c}\omega^{0} \\
\delta(u)\end{array}$ & $\begin{array}{c}\omega \\
\delta^{\prime}(u)\end{array}$ & $\cdots$ & & \\
\hline $\begin{array}{l}\text { 1-loop } \\
\mathcal{O}\left(G^{2}\right)\end{array}$ & $\ln \omega$ & $\begin{array}{l}\omega^{0} \\
\delta(u)\end{array}$ & $\begin{array}{l}\omega \operatorname{spin} \text { dependent } \\
u^{-2}\end{array}$ & $\begin{array}{c}\omega \\
\delta^{\prime}(u)\end{array}$ & $\omega^{2} \ln ^{-3} \omega$ & $\cdots$ \\
\hline $\begin{array}{l}\text { 2-loop } \\
\mathcal{O}\left(G^{3}\right)\end{array}$ & $\begin{array}{c}\omega(\ln \omega)^{2} \\
u^{-2} \ln u\end{array}$ & $\omega \ln \omega$ & $\begin{array}{c}\omega \\
\delta^{\prime}(u)\end{array}$ & $\begin{array}{c}\omega^{2}(\ln \omega)^{2} \\
u^{-3} \ln u\end{array}$ & $\begin{array}{c}\omega^{2} \ln \omega \\
u^{-3}\end{array}$ & $\cdots$ \\
\hline $\begin{array}{c}\text { n-loop } \\
\mathcal{O}\left(G^{n+1}\right)\end{array}$ & $\begin{array}{l}\omega^{n-1}(\ln \omega)^{n} \\
u^{-n}(\ln u)^{n-1}\end{array}$ & $\begin{array}{l}\omega^{n-1}(\ln \omega)^{n-1} \\
u^{-n}(\ln u)^{n-2}\end{array}$ & $\cdots$ & & & \\
\hline
\end{tabular}

FIG. 2. This table summarizes different orders of low frequency gravitational waveform $\tilde{e}^{\mu \nu}(\omega, R, \mathbf{n})$ in the $\omega \rightarrow 0$ limit (written in green) and their relations to post-Minkowskian (PM) expansion. It also describes how the late and early time gravitational waveforms $e^{\mu \nu}(u, R \hat{n})$ behave at large retarded time $u$ (written in blue). The spin-dependent order $\omega \ln \omega$ gravitational waveform indicated inside the blue cell is the primary interest of this paper.

$$
\begin{aligned}
& h_{\mu \nu}(x) \equiv \frac{1}{2}\left(g_{\mu \nu}(x)-\eta_{\mu \nu}\right), \\
& e_{\mu \nu}(x) \equiv h_{\mu \nu}(x)-\frac{1}{2} \eta_{\mu \nu} \eta^{\alpha \beta} h_{\alpha \beta}(x) .
\end{aligned}
$$

The time Fourier transform of the trace reversed metric is defined as

$$
\tilde{e}^{\mu \nu}(\omega, \vec{x})=\int_{-\infty}^{\infty} d t e^{i \omega t} e^{\mu \nu}(t, \vec{x})
$$

With this scattering setup the goal is to determine the radiative mode of $\tilde{e}^{\mu \nu}(\omega, R \hat{n})$ in the frequency range $R^{-1} \ll \omega \ll L^{-1}$. In $[13,14]$ the first three nonanalytic contributions in the $\omega \rightarrow 0$ limit have been evaluated, which behave like $\omega^{-1}, \ln \omega$, and $\omega(\ln \omega)^{2}$, and the coefficients depend only on the incoming and outgoing momenta and $\hat{n}$. These low-frequency gravitational waveforms contribute to static gravitational memory, $u^{-1}$ tail memory, and $u^{-2} \ln u$ tail memory, respectively. To derive these results the authors developed an iterative prescription of solving the geodesic equation of scattered objects and Einstein equation treating gravitational constant $G$ as an iterative parameter. Since $G M \omega$ is a dimensionless quantity in the unit where speed of light is unity, expansion in the power of $\omega$ is equivalent to expansion in the power of $G$, where $M$ represents the mass or momentum of scattered objects. Generalizing this prescription the structure of the leading nonanalytic contribution in each iterative order has been conjectured in [14], which has been summarized in the second column of the table given in Fig. 2.

If the scattered objects carry spin $\Sigma$, along with $G M \omega$ there will be another dimensionless quantity $G \Sigma \omega^{2}$. This simple dimensional analysis tells us that the spin dependence of the gravitational waveform at any iterative order in $G$ will carry an extra factor of $\omega$ relative to the spin-independent leading nonanalytic contribution at that order. With this observation, from the table in Fig. 2 we see that at the order $G$ of the gravitational waveform, spin dependence appears at order $\omega^{0}$. Since the order $\omega^{0}$ term is analytic in the $\omega \rightarrow 0$ limit, it does not contribute to a displacement kind of memory [18]. Now in the next iterative order, i.e., in the order $G^{2}$ of the gravitational waveform, the spin dependence comes at order $\omega \ln \omega$, which is nonanalytic in the $\omega \rightarrow 0$ limit and contributes to order $u^{-2}$ tail memory. ${ }^{1}$ The existence of spin-dependent $u^{-2}$ tail memory was first pointed out in the Sec. (5.3) of [14], using some naive analysis of the matter and gravitational energy-momentum tensor, indicated within the blue cell in the table of Fig. 2. From the table, it is also clear that the spin-dependent order $\omega \ln \omega$ waveform is not exact, but it receives corrections from order $G^{3}$ as well. The order $G^{3}$ correction to the $\omega \ln \omega$ gravitational waveform is expected to be spin independent.

In this paper, our main goal is to derive the $\omega \ln \omega$ gravitational waveform at order $G^{2}$ and the gravitational tail memory it predicts. The main result of the paper is summarized in Eqs. (2.9) and (2.10). In Sec. II we make a conjecture on the $\omega \ln \omega$ gravitational waveform from the classical limit of the sub-subleading soft graviton theorem and discuss various applications of the result in different limits. Then in Sec. III we derive the $\omega \ln \omega$ gravitational waveform directly for a classical scattering process without any reference to the soft graviton theorem. Various

\footnotetext{
${ }^{1}$ Here we want to emphasize that the order $u^{-2}$ tail memory is there even for the scattering of nonspinning objects as $G \mathbf{L} \omega^{2}$ for $\mathbf{L}$ being the orbital angular momentum of the scattered object, and is also a dimensionless quantity such as $G \Sigma \omega^{2}$. Hence in our final result of order $u^{-2}$ tail memory, setting the spins of the scattered objects to zero, one can read off the result of the gravitational waveform for the nonspinning object's scattering.
} 
appendixes discuss intermediate steps required in the analysis of Sec. III. Finally, in Sec. IV we conclude the paper by discussing the novel features of our result, its theoretical and observational importance, and the possibility of rederivation of our result with other available prescriptions in the literature.

\section{PREDICTION OF SPIN-DEPENDENT GRAVITATIONAL WAVEFORM FROM CLASSICAL LIMIT OF SOFT GRAVITON THEOREM}

Emboldened by the success of predicting a long wavelength gravitational waveform and gravitational tail memory from the classical limit of the quantum soft graviton theorem at subleading and sub-subleading orders [12,13], here we proceed to conjecture the leading spin-dependent gravitational tail memory in $D=4$ spacetime dimensions.

In spacetime dimensions $D>4$, the universal piece of the quantum soft graviton operator up to sub-subleading order takes the following form [19]:

$\mathbf{S}_{\mathrm{uni}}^{g r}=\sum_{a=1}^{M+N}\left[\frac{\varepsilon_{\mu \nu} p_{a}^{\mu} p_{a}^{\nu}}{p_{a} \cdot k}+\frac{\varepsilon_{\mu \nu} p_{a}^{\mu} k_{\rho} \hat{\mathbf{J}}_{a}^{\rho \nu}}{p_{a} \cdot k}+\frac{1}{2} \frac{\varepsilon_{\mu \nu} k_{\rho} k_{\sigma} \hat{\mathbf{J}}_{a}^{\rho \mu} \hat{\mathbf{J}}_{a}^{\sigma \nu}}{p_{a} \cdot k}\right]$.

Note that we have not included the nonuniversal piece in the sub-subleading soft factor, which is determined in terms of nonminimal coupling of the hard particle field to the soft graviton field via the Riemann tensor along with the three point 1 PI vertex involving two hard particles and a soft graviton [19]. In the above expression of the soft graviton operator $\varepsilon$ represents the polarization tensor of the soft graviton with momentum $k=\omega \mathbf{n}$, where $\omega$ is the energy of the outgoing soft graviton. The scattering process involves $M$ number of ingoing hard particles and $N$ number of outgoing hard particles. The momentum of hard particle $a$ is denoted by $p_{a}$, and the angular momentum operator is given by

$$
\hat{\mathbf{J}}_{a}^{\mu \nu} \equiv p_{a}^{\nu} \frac{\partial}{\partial p_{a \mu}}-p_{a}^{\mu} \frac{\partial}{\partial p_{a \nu}}+\hat{\boldsymbol{\Sigma}}_{a}^{\mu \nu},
$$

where $\hat{\boldsymbol{\Sigma}}_{a}^{\mu \nu}$ represents the quantum spin generator of the Lorentz group for hard particle $a$. We are following the convention that the outgoing particles carry positive energy and the incoming particles carry negative energy.

From the classical limit of the quantum soft graviton theorem up to sub-subleading order in $D=4$, we get the following radiative mode of the gravitational waveform with frequency $\omega$ at distance $R=|\vec{x}|$ from the scattering center $[12,16,17]$ :

$$
\begin{aligned}
\tilde{e}^{\mu \nu}(\omega, \vec{x})= & (-i) \frac{2 G}{R} e^{i \omega R} \exp \left[-2 i G \ln \{(\omega+i \epsilon) R\} \sum_{b=1}^{N} p_{b} \cdot k\right] \\
& \times \sum_{a=1}^{M+N}\left[\frac{p_{a}^{\mu} p_{a}^{\nu}}{p_{a} \cdot k}-i \frac{p_{a}^{(\mu} k_{\rho} \mathbf{J}_{a}^{\rho \nu)}}{p_{a} \cdot k}-\frac{1}{2} \frac{\varepsilon_{\mu \nu} k_{\rho} k_{\sigma} \mathbf{J}_{a}^{\rho \mu} \mathbf{J}_{a}^{\sigma \nu}}{p_{a} \cdot k}\right]
\end{aligned}
$$

where $\mathbf{J}_{a}^{\mu \nu}$ is the classical angular momentum of particle $a$ expressed in terms of trajectory $X_{a}$ and classical spin $\Sigma_{a}$ of particle $a$ by the following relation:

$$
\mathbf{J}_{a}^{\mu \nu}=X_{a}^{\mu} p_{a}^{\nu}-X_{a}^{\nu} p_{a}^{\mu}+\Sigma_{a}^{\mu \nu}
$$

In Eq. (2.3), the symmetrization convention we are following is $A^{(\alpha} B^{\beta)} \equiv \frac{1}{2}\left(A^{\alpha} B^{\beta}+A^{\beta} B^{\alpha}\right)$. It is well known that in four spacetime dimensions the asymptotic trajectories of the scattered particles receive logarithmic correction due to a long range gravitational force [12,17] [e.g., if the asymptotic trajectory of particle $a$ is represented by $X_{a}^{\mu}(\sigma)=r_{a}^{\mu}+v_{a}^{\mu} \sigma+Y_{a}^{\mu}(\sigma)$ for proper time $\sigma$, then $Y_{a}(\sigma)$ behaves as $\ln |\sigma|$ for $\sigma \rightarrow \pm \infty]$. So following the prescription of $[12,17]$, if we replace $\ln |\sigma|$ by $-\ln \left(\omega+i \epsilon \eta_{a}\right)$ in the classical angular momentum of Eq. (2.3), it predicts the correct gravitational waveform, which has been independently verified in $[13,14]$ up to sub-subleading order nonspinning particle scattering. Here once again using the same prescription, from Eq. (2.3) we get

$$
\begin{aligned}
\tilde{e}^{\mu \nu}(\omega, \vec{x})= & (-i) \frac{2 G}{R} e^{i \omega R} \exp \left[-2 i G \ln \{(\omega+i \epsilon) R\} \sum_{b=1}^{N} p_{b} \cdot k\right] \\
& \times\left[\sum_{a=1}^{M+N} \frac{p_{a}^{\mu} p_{a}^{\nu}}{p_{a} \cdot k}+\sum_{a=1}^{M+N} \frac{p_{a}^{(\mu} k_{\rho}}{p_{a} \cdot k}\left\{\left(p_{a}^{\nu)} \frac{\partial}{\partial p_{a \rho}}-p_{a}^{\rho} \frac{\partial}{\left.\partial p_{a \nu}\right)}\right) K_{g r}^{c l}-i\left(r_{a}^{\rho} p_{a}^{\nu)}-r_{a}^{\nu)} p_{a}^{\rho}+\Sigma_{a}^{\rho \nu)}\right)\right\}\right. \\
& +\frac{1}{2} \sum_{a=1}^{M+N} \frac{k_{\rho} k_{\sigma}}{p_{a} \cdot k}\left\{\left(p_{a}^{\mu} \frac{\partial}{\partial p_{a \rho}}-p_{a}^{\rho} \frac{\partial}{\partial p_{a \mu}}\right) K_{g r}^{c l}-i\left(r_{a}^{\rho} p_{a}^{\mu}-r_{a}^{\mu} p_{a}^{\rho}+\Sigma_{a}^{\rho \mu}\right)\right\} \\
& \left.\times\left\{\left(p_{a}^{\nu} \frac{\partial}{\partial p_{a \sigma}}-p_{a}^{\sigma} \frac{\partial}{\partial p_{a \nu}}\right) K_{g r}^{c l}-i\left(r_{a}^{\sigma} p_{a}^{\nu}-r_{a}^{\nu} p_{a}^{\sigma}+\Sigma_{a}^{\sigma \nu}\right)\right\}\right]
\end{aligned}
$$


where the expression of $K_{g r}^{c l}$ is given by [14]

$$
\begin{aligned}
K_{g r}^{c l} & =-\frac{i}{2}(8 \pi G) \sum_{\substack{b, c \\
b \neq c}} \int_{\omega}^{L^{-1}} \frac{d^{4} \ell}{(2 \pi)^{4}} G_{r}(\ell) \frac{1}{p_{b} \cdot \ell+i \epsilon} \frac{1}{p_{c} \cdot \ell-i \epsilon}\left\{\left(p_{b} \cdot p_{c}\right)^{2}-\frac{1}{2} p_{b}^{2} p_{c}^{2}\right\} \\
& =-\frac{i}{2}(2 G) \sum_{\substack{b, c \\
b \neq c \\
\eta_{b} c=1}} \ln \left\{L\left(\omega+i \epsilon \eta_{b}\right)\right\} \frac{\left(p_{b} \cdot p_{c}\right)^{2}-\frac{1}{2} p_{b}^{2} p_{c}^{2}}{\sqrt{\left(p_{b} \cdot p_{c}\right)^{2}-p_{b}^{2} p_{c}^{2}}}
\end{aligned}
$$

In the above expression $\eta_{b}=+1$ if particle $b$ is outgoing and $\eta_{b}=-1$ if particle $b$ is ingoing in the scattering event. In the expression of $K_{g r}^{c l}, L^{-1}$ denotes the UV energy scale, and for the value of $\omega$ below this UV scale our result for the gravitational waveform can be trusted. Roughly, the length scale $L \simeq\left|r_{a}-r_{b}\right|$ is of the order of the impact parameters for a scattering event involving unbounded hyperbolic orbits of compact objects.

Now expanding the exponential in the small $\omega$ limit we find the following order $\mathcal{O}(\omega \ln \omega)$ contribution of the gravitational waveform at order $G^{2}$ :

$$
\begin{aligned}
\Delta_{\left(G^{2}\right)}^{(\omega \ln \omega)} \tilde{e}^{\mu \nu}(\omega, \vec{x})= & (-i) \frac{2 G}{R} \exp \left\{i \omega R-2 i G \ln R \sum_{b=1}^{N} p_{b} \cdot k\right\}\left[-2 G \ln \{\omega+i \epsilon\} \sum_{b=1}^{N} p_{b} \cdot k\right. \\
& \times \sum_{a=1}^{M+N} \frac{p_{a}^{(\mu} k_{\rho}}{p_{a} \cdot k}\left(r_{a}^{\rho} p_{a}^{\nu)}-r_{a}^{\nu} p_{a}^{\rho}+\Sigma_{a}^{\rho \nu)}\right) \\
& -\frac{i}{2} \sum_{a=1}^{M+N} \frac{k_{\rho} k_{\sigma}}{p_{a} \cdot k}\left\{\left(p_{a}^{\mu} \frac{\partial}{\partial p_{a \rho}}-p_{a}^{\rho} \frac{\partial}{\partial p_{a \mu}}\right) K_{g r}^{c l} \times\left(r_{a}^{\sigma} p_{a}^{\nu}-r_{a}^{\nu} p_{a}^{\sigma}+\Sigma_{a}^{\sigma \nu}\right)\right. \\
& \left.\left.+\left(p_{a}^{\nu} \frac{\partial}{\partial p_{a \sigma}}-p_{a}^{\sigma} \frac{\partial}{\partial p_{a \nu}}\right) K_{g r}^{c l} \times\left(r_{a}^{\rho} p_{a}^{\mu}-r_{a}^{\mu} p_{a}^{\rho}+\Sigma_{a}^{\rho \mu}\right)\right\}\right] .
\end{aligned}
$$

Explicitly the above equation can be written in terms of incoming scattering data $\left\{p_{a}^{\prime}, \Sigma_{a}^{\prime}, r_{a}^{\prime}\right\}$ and outgoing scattering data $\left\{p_{a}, \Sigma_{a}, r_{a}\right\}$ in the following way:

$$
\begin{aligned}
\Delta_{\left(G^{2}\right)}^{(\omega \ln \omega)} \tilde{e}^{\mu \nu}(\omega, R, \mathbf{n})= & \frac{G^{2}}{R} \exp \left\{i \omega R-2 i G \omega \ln R \sum_{b=1}^{N} p_{b} \cdot \mathbf{n}\right\} \\
& \times\left[4 i \omega \operatorname { l n } ( \omega + i \epsilon ) \sum _ { b = 1 } ^ { N } p _ { b } \cdot \mathbf { n } \left\{\sum_{a=1}^{N} \frac{p_{a}^{(\mu} \mathbf{n}_{\rho}}{p_{a} \cdot \mathbf{n}}\left(r_{a}^{\rho} p_{a}^{\nu)}-r_{a}^{\nu)} p_{a}^{\rho}+\Sigma_{a}^{\rho \nu)}\right)\right.\right. \\
& \left.-\sum_{a=1}^{M} \frac{p_{a}^{\prime(\mu} \mathbf{n}_{\rho}}{p_{a}^{\prime} \cdot \mathbf{n}}\left(r_{a}^{\prime \rho} p_{a}^{\prime \nu)}-r_{a}^{\prime \nu)} p_{a}^{\prime \rho}+\Sigma_{a}^{\prime \rho \nu}\right)\right\} \\
& +i \omega \ln (\omega+i \epsilon) \sum_{a=1}^{N} \sum_{b=1}^{N} \frac{p_{a} \cdot p_{b}}{\left[\left(p_{a} \cdot p_{b}\right)^{2}-p_{a}^{2} p_{b}^{2}\right]^{3 / 2}}\left\{2\left(p_{a} \cdot p_{b}\right)^{2}-3 p_{a}^{2} p_{b}^{2}\right\} \frac{\mathbf{n}_{\rho} \mathbf{n}_{\sigma}}{p_{a} \cdot \mathbf{n}} \\
& \times\left\{\left(p_{a}^{\mu} p_{b}^{\rho}-p_{a}^{\rho} p_{b}^{\mu}\right)\left(r_{a}^{\sigma} p_{a}^{\nu}-r_{a}^{\nu} p_{a}^{\sigma}+\Sigma_{a}^{\sigma \nu}\right)+\left(p_{a}^{\nu} p_{b}^{\sigma}-p_{a}^{\sigma} p_{b}^{\nu}\right)\left(r_{a}^{\rho} p_{a}^{\mu}-r_{a}^{\mu} p_{a}^{\rho}+\Sigma_{a}^{\rho \mu}\right)\right\} \\
& +i \omega \ln (\omega-i \epsilon) \sum_{a=1}^{M} \sum_{\substack{b=1 \\
b \neq a}}^{M} \frac{p_{a}^{\prime} \cdot p_{b}^{\prime}}{\left[\left(p_{a}^{\prime} \cdot p_{b}^{\prime}\right)^{2}-p_{a}^{\prime 2} p_{b}^{\prime 2}\right]^{3 / 2}}\left\{2\left(p_{a}^{\prime} \cdot p_{b}^{\prime}\right)^{2}-3 p_{a}^{\prime 2} p_{b}^{\prime 2}\right\} \frac{\mathbf{n}_{\rho} \mathbf{n}_{\sigma}}{p_{a}^{\prime} \cdot \mathbf{n}} \\
& \left.\times\left\{\left(p_{a}^{\prime \mu} p_{b}^{\prime \rho}-p_{a}^{\prime \rho} p_{b}^{\prime \mu}\right)\left(r_{a}^{\prime \sigma} p_{a}^{\prime \nu}-r_{a}^{\prime \nu} p_{a}^{\prime \sigma}+\Sigma_{a}^{\prime \sigma \nu}\right)+\left(p_{a}^{\prime \nu} p_{b}^{\prime \sigma}-p_{a}^{\prime \sigma} p_{b}^{\prime \nu}\right)\left(r_{a}^{\prime \rho} p_{a}^{\prime \mu}-r_{a}^{\prime \mu} p_{a}^{\prime \rho}+\Sigma_{a}^{\prime \rho \mu}\right)\right\}\right]
\end{aligned}
$$

where $k^{\mu}=\omega \mathbf{n}^{\mu}=\omega(1, \hat{n})$ with $\hat{n}$ being the unit vector denoting the direction of gravitational radiation. For the scattering of nonspinning objects, we can read off the order $\omega \ln \omega$ gravitational waveform after setting $\Sigma_{a}=0$ and $\Sigma_{a}^{\prime}=0$ in the above relation. 


\section{A. Spin-dependent tail memory}

Performing a Fourier transform in the $\omega$ variable of the expression in Eq. (2.8), we find the following expressions for late and early time gravitational waveforms:

$$
\begin{aligned}
\Delta_{\left(G^{2}\right)}^{\left(1 / u^{2}\right)} e^{\mu \nu}(u, \vec{x}=R \hat{n})= & -\frac{G^{2}}{R} \frac{1}{u^{2}}\left[4 \sum _ { b = 1 } ^ { N } p _ { b } \cdot \mathbf { n } \left\{\sum_{a=1}^{N} \frac{p_{a}^{(\mu} \mathbf{n}_{\rho}}{p_{a} \cdot \mathbf{n}}\left(r_{a}^{\rho} p_{a}^{\nu)}-r_{a}^{\nu)} p_{a}^{\rho}+\Sigma_{a}^{\rho \nu)}\right)\right.\right. \\
& \left.-\sum_{a=1}^{M} \frac{p_{a}^{\prime(\mu} \mathbf{n}_{\rho}}{p_{a}^{\prime} \cdot \mathbf{n}}\left(r_{a}^{\prime \rho} p_{a}^{\prime \nu)}-r_{a}^{\prime \nu)} p_{a}^{\prime \rho}+\Sigma_{a}^{\prime \rho \nu)}\right)\right\} \\
& +\sum_{a=1}^{N} \sum_{\substack{b=1 \\
b \neq a}}^{N} \frac{p_{a} \cdot p_{b}}{\left[\left(p_{a} \cdot p_{b}\right)^{2}-p_{a}^{2} p_{b}^{2}\right]^{3 / 2}}\left\{2\left(p_{a} \cdot p_{b}\right)^{2}-3 p_{a}^{2} p_{b}^{2}\right\} \frac{\mathbf{n}_{\rho} \mathbf{n}_{\sigma}}{p_{a} \cdot \mathbf{n}}\left\{\left(p_{a}^{\mu} p_{b}^{\rho}-p_{a}^{\rho} p_{b}^{\mu}\right)\left(r_{a}^{\sigma} p_{a}^{\nu}-r_{a}^{\nu} p_{a}^{\sigma}+\Sigma_{a}^{\sigma \nu}\right)\right. \\
& \left.\left.+\left(p_{a}^{\nu} p_{b}^{\sigma}-p_{a}^{\sigma} p_{b}^{\nu}\right)\left(r_{a}^{\rho} p_{a}^{\mu}-r_{a}^{\mu} p_{a}^{\rho}+\Sigma_{a}^{\rho \mu}\right)\right\}\right], \quad \text { for } u \rightarrow+\infty \\
\Delta_{\left(G^{2}\right)}^{\left(1 / u^{2}\right)} e^{\mu \nu}(u, \vec{x}=R \hat{n})= & \frac{G^{2}}{R} \frac{1}{u^{2}} \sum_{a=1}^{M} \sum_{b=1}^{M} \frac{p_{a}^{\prime} \cdot p_{b}^{\prime}}{\left[\left(p_{a}^{\prime} \cdot p_{b}^{\prime}\right)^{2}-p_{a}^{\prime 2} p_{b}^{\prime 2}\right]^{3 / 2}}\left\{2\left(p_{a}^{\prime} \cdot p_{b}^{\prime}\right)^{2}-3 p_{a}^{\prime 2} p_{b}^{\prime 2}\right\} \frac{\mathbf{n}_{\rho} \mathbf{n}_{\sigma}}{p_{a}^{\prime} \cdot \mathbf{n}} \\
& \times\left\{\left(p_{a}^{\prime \mu} p_{b}^{\prime \rho}-p_{a}^{\prime \rho} p_{b}^{\prime \mu}\right)\left(r_{a}^{\prime \sigma} p_{a}^{\prime \nu}-r_{a}^{\prime \nu} p_{a}^{\prime \sigma}+\Sigma_{a}^{\prime \sigma \nu}\right)+\left(p_{a}^{\prime \prime} p_{b}^{\prime \sigma}-p_{a}^{\prime \sigma} p_{b}^{\prime \nu}\right)\left(r_{a}^{\prime \rho} p_{a}^{\prime \mu}-r_{a}^{\prime \mu} p_{a}^{\prime \rho}+\Sigma_{a}^{\prime \rho \mu}\right)\right\} \\
& \text { for } u \rightarrow-\infty,
\end{aligned}
$$

where retarded time $u$ is given by $u=t-R+$ $2 G \ln R \sum_{b=1}^{N} p_{b} \cdot \mathbf{n}$. The above expressions predict the order $u^{-2}$ tail memory along with the known order $u^{-1}$ and $u^{-2} \ln u$ tail memories. For nonspinning object scattering the order $\mathcal{O}\left(u^{-2}\right)$ tail memory is nonvanishing and can be read off by setting $\Sigma_{a}=0$ and $\Sigma_{a}^{\prime}=0$ in the above expressions. At this stage the order $\mathcal{O}\left(u^{-2}\right)$ gravitational tail memory seems to be nonuniversal even at order $G^{2}$, as the result depends not only on the asymptotic data, i.e., incoming and outgoing momenta and spins of scattered objects, but also on the choice of the region $\mathcal{R}$ through $r_{a}$. Let us fix two particular time slices before and after the scattering event such that before and after this time, the value of the kinetic energy of all the particles exceeds the value of their potential energy due to interaction among themselves. This way we can fix the boundary of the region $\mathcal{R}$. In our notation this boundary corresponds to $\sigma=0$ for the particle's trajectory, i.e., $X_{a}(\sigma=0)=r_{a}$. Now if we want to change our definition of region $\mathcal{R}$ to a different time slice, say $\sigma=\sigma_{0}$, it would not affect our result above as under this choice $r_{a}$ will be shifted by $r_{a}+\frac{p_{a}}{m_{a}} \sigma_{0}$ and that does not affect the $\left(r_{a}^{\alpha} p_{a}^{\beta}-r_{a}^{\beta} p_{a}^{\alpha}\right)$ combination. Hence this property suggests that in principle, it is possible to write the result only in terms of the asymptotic data. As a piece of evidence, consider a special case of the $2 \rightarrow 2$ scattering event where object- 1 is very heavy and object- 2 is a probe of small mass, and suppose they scatter with a large impact parameter. Now for this process, if we choose the scattering center at the origin of object- 1 , then $r_{1}=0$ and $r_{2}$ is the impact parameter which is determinable in terms of asymptotic scattering data.

\section{B. Spin-dependent tail memory rewritten}

Consider a classical scattering process where out of $N$ outgoing objects the $\tilde{N}$ number of objects is massive and the rest are massless radiation including the gravitational wave. For this process in [15], it has been shown that the late time gravitational memory at order $u^{-1}$ and $u^{-2} \ln u$ can be fully expressed in terms of the momenta of incoming massive objects, incoming massless radiation, and outgoing massive objects only. So the information about outgoing massless radiation is not needed to compute the late time gravitational memory at order $u^{-1}$ and $u^{-2} \ln u$. In this subsection, we show that the late time gravitational memory at order $u^{-2}$, given in Eq. (2.9), can also be rewritten in such a way that it would not carry any information of outgoing massless radiation.

We denote the momenta of outgoing massive objects by $\tilde{p}_{a}$, the spins of outgoing massive objects by $\tilde{\Sigma}_{a}$, and the coordinates of the intersection points with the boundary of region $\mathcal{R}$ of outgoing massive objects by $\tilde{r}_{a}$ for $a=1, \ldots, \tilde{N}$. Then after some manipulation using conservation of asymptotic momenta and asymptotic angular momenta the expression in Eq. (2.9) can be rewritten as 


$$
\begin{aligned}
& \Delta_{\left(G^{2}\right)}^{\left(1 / u^{2}\right)} e^{\mu \nu}(u, \vec{x}=R \hat{n})=-\frac{G^{2}}{R} \frac{1}{u^{2}}\left[4 \sum_{b=1}^{\tilde{N}} \tilde{p}_{b} \cdot \mathbf{n}\left\{\sum_{a=1}^{\tilde{N}} \frac{\tilde{p}_{a}^{(\mu} \mathbf{n}_{\rho}}{\tilde{p}_{a} \cdot \mathbf{n}}\left(\tilde{r}_{a}^{\rho} \tilde{p}_{a}^{\nu)}-\tilde{r}_{a}^{\nu)} \tilde{p}_{a}^{\rho}+\tilde{\Sigma}_{a}^{\rho \nu)}\right)\right\}\right. \\
& -4\left(P^{\prime} \cdot \mathbf{n}\right)\left\{\sum_{a=1}^{M} \frac{p_{a}^{\prime(\mu} \mathbf{n}_{\rho}}{p_{a}^{\prime} \cdot \mathbf{n}}\left(r_{a}^{\prime \rho} p_{a}^{\prime \nu)}-r_{a}^{\prime \nu)} p_{a}^{\prime \rho}+\Sigma_{a}^{\prime \rho \nu)}\right)\right\} \\
& +\sum_{a=1}^{\tilde{N}} \sum_{\substack{b=1 \\
b \neq a}}^{\tilde{N}} \frac{\tilde{p}_{a} \cdot \tilde{p}_{b}}{\left[\left(\tilde{p}_{a} \cdot \tilde{p}_{b}\right)^{2}-\tilde{p}_{a}^{2} \tilde{p}_{b}^{2}\right]^{3 / 2}}\left\{2\left(\tilde{p}_{a} \cdot \tilde{p}_{b}\right)^{2}-3 \tilde{p}_{a}^{2} \tilde{p}_{b}^{2}\right\} \frac{\mathbf{n}_{\rho} \mathbf{n}_{\sigma}}{\tilde{p}_{a} \cdot \mathbf{n}}\left\{\left(\tilde{p}_{a}^{\mu} \tilde{p}_{b}^{\rho}-\tilde{p}_{a}^{\rho} \tilde{p}_{b}^{\mu}\right)\left(\tilde{r}_{a}^{\sigma} \tilde{p}_{a}^{\nu}-\tilde{r}_{a}^{\nu} \tilde{p}_{a}^{\sigma}+\tilde{\Sigma}_{a}^{\sigma \nu}\right)\right. \\
& \left.+\left(\tilde{p}_{a}^{\nu} \tilde{p}_{b}^{\sigma}-\tilde{p}_{a}^{\sigma} \tilde{p}_{b}^{\nu}\right)\left(\tilde{r}_{a}^{\rho} \tilde{p}_{a}^{\mu}-\tilde{r}_{a}^{\mu} \tilde{p}_{a}^{\rho}+\tilde{\Sigma}_{a}^{\rho \mu}\right)\right\} \\
& -4 \tilde{P}^{(\mu} \mathbf{n}_{\rho} \sum_{a=1}^{\tilde{N}}\left(\tilde{r}_{a}^{\rho} \tilde{p}_{a}^{\nu)}-\tilde{r}_{a}^{\nu)} \tilde{p}_{a}^{\rho}+\tilde{\Sigma}_{a}^{\rho \nu)}\right) \\
& \left.+4 P^{\prime(\mu} \mathbf{n}_{\rho} \sum_{a=1}^{M}\left(r_{a}^{\prime \rho} p_{a}^{\prime \nu)}-r_{a}^{\prime \nu)} p_{a}^{\prime \rho}+\Sigma_{a}^{\prime \rho \nu)}\right)\right], \quad \text { for } u \rightarrow+\infty
\end{aligned}
$$

where $P^{\prime \mu}=\sum_{a=1}^{M} p_{a}^{\prime \mu}$ and $\tilde{P}^{\mu}=\sum_{a=1}^{\tilde{N}} \tilde{p}_{a}^{\mu}$. The above expression does not carry any information about massless outgoing radiation.

Now consider the binary black hole merger process, where there is only one incoming object (i.e., $M=1$ ) that is the bound state of two black holes, and in the final state there is one massive black hole (i.e., $\tilde{N}=1$ ) and lots of gravitational radiation. Hence for this process the third and fourth lines after the equality vanish in Eq. (2.11). Also the first term within the square bracket cancels the second to last term as well as the second term within the square bracket cancels the last term in (2.11). Hence the late time gravitational memory at order $G^{2} u^{-2}$ given in (2.11) vanishes for the black hole merger process. But as discussed in the Introduction, the $u^{-2}$ tail memory receives correction at order $G^{3}$, which has not been derived or conjectured yet. So we do not know whether the order $G^{3} u^{-2}$ memory also vanishes or not for the black hole merger process.

Now let us consider a gravitational scattering process where all the incoming and outgoing objects are massless particles/radiation. For this event after using conservation of asymptotic momenta and asymptotic angular momenta, the expression in Eqs. (2.9) and (2.10) takes the following simple form:

$$
\begin{aligned}
& \Delta_{\left(G^{2}\right)}^{\left(1 / u^{2}\right)} e^{\mu \nu}(u \rightarrow+\infty, \vec{x}=R \hat{n})=-\Delta_{\left(G^{2}\right)}^{\left(1 / u^{2}\right)} e^{\mu \nu}(u \rightarrow-\infty, \vec{x}=R \hat{n}) \\
& =-\frac{4 G^{2}}{R} \frac{1}{u^{2}}\left[P^{\prime(\mu} \mathbf{n}_{\rho}\left(r_{a}^{\prime \rho} p_{a}^{\prime \nu)}-r_{a}^{\prime \nu)} p_{a}^{\prime \rho}+\Sigma_{a}^{\prime \rho \nu)}\right)-\left(P^{\prime} \cdot \mathbf{n}\right) \sum_{a=1}^{M} \frac{p_{a}^{\prime(\mu} \mathbf{n}_{\rho}}{p_{a}^{\prime} \cdot \mathbf{n}}\left(r_{a}^{\prime \rho} p_{a}^{\prime \nu)}-r_{a}^{\prime \nu)} p_{a}^{\prime \rho}+\Sigma_{a}^{\prime \rho \nu)}\right)\right]
\end{aligned}
$$

From the above expression it is clear that for massless particle scattering the order $\mathcal{O}\left(G^{2} u^{-2}\right)$ gravitational tail memory is completely determined in terms of the scattering data of ingoing particles only. So the result is independent of scattering angles even if the ingoing massless particles/ radiation form a black hole along with some massless gravitational radiation in the final state. In [15] this feature has been established for $u^{-1}$ and $u^{-2} \ln u$ tail memories as well.

\section{DERIVATION OF SPIN-DEPENDENT GRAVITATIONAL WAVEFORM}

We consider a classical scattering process where the $M$ number of spinning macroscopic objects come in from the asymptotic past, go through some complicated process involving fusion, splitting, etc., within a finite region of spacetime $\mathcal{R}$, and finally disperse to $N$ number of objects including finite energy radiation flux as shown in Fig. 1. For this kind of scattering event, we are interested in determining the late and early time gravitational waveform, which is also related to the radiative mode of low-frequency gravitational waveform via Fourier transformation in the time variable. We choose the region $\mathcal{R}$ to be sufficiently large so that outside this region only long-range gravitational interaction is present. Let us consider the size of the spacetime region $\mathcal{R}$ to be $L$. Now we choose the scattering center well inside the region $\mathcal{R}$ and place the gravitational wave detector at a distance $R$ from the scattering center along the direction $\hat{n}$. With this setup we want to determine 
the $\frac{1}{R}$ component of the gravitational waveform with frequency $\omega$ within the range $R^{-1} \ll \omega \ll L^{-1}$.

We proceed by defining the deviation of the metric from a flat background, and it is a trace reversed component in the following way:

$$
\begin{aligned}
h_{\mu \nu}(x) & \equiv \frac{1}{2}\left(g_{\mu \nu}(x)-\eta_{\mu \nu}\right), \\
e_{\mu \nu}(x) & \equiv h_{\mu \nu}(x)-\frac{1}{2} \eta_{\mu \nu} \eta^{\alpha \beta} h_{\alpha \beta}(x) .
\end{aligned}
$$

In the above expression $\eta$ represents the Minkowski metric in a mostly positive signature. In several literatures including $[13,16,17,20,21]$ the following relation between the radiative mode of the gravitational waveform and the Fourier transform of the total energy-momentum tensor has been derived in four spacetime dimensions:

$$
\tilde{e}^{\mu \nu}(\omega, R, \hat{n}) \simeq \frac{2 G}{R} e^{i \omega R} \hat{T}^{\mu \nu}(k),
$$

where under the $\simeq$ sign, we are neglecting the terms with higher powers of $R^{-1}$. In the relation above $\vec{x}=R \hat{n}, k=$ $\omega(1, \hat{n}) \equiv \omega \mathbf{n}$ and the gravitational radiation is considered to be outgoing. The expressions of $\tilde{e}^{\mu \nu}$ and $\hat{T}^{\mu \nu}$ are given by

$$
\tilde{e}^{\mu \nu}(\omega, \vec{x})=\int_{-\infty}^{\infty} d t e^{i \omega t} e^{\mu \nu}(t, \vec{x}),
$$

$\hat{T}^{\mu \nu}(k)=\int d^{4} x e^{-i k \cdot x} T^{\mu \nu}(x)+$ boundary terms at $\infty$,

where $T^{\mu \nu}(x)$ is the total (matter + gravitational) energymomentum tensor that appears in the right-hand side (RHS) of linearized Einstein's equation. In the above relation the Fourier transform of the energy-momentum tensor is defined inside the region $|\vec{x}| \ll R$ or equivalently we may need to add appropriate boundary terms at $\infty$ to make the integral well defined $[13,16,17,21]$.

\section{A. General setup and strategy}

Consider the incoming particles have masses $\left\{m_{a}^{\prime}\right\}$, velocities $\left\{v_{a}^{\prime}\right\}$, momenta $\left\{p_{a}^{\prime}=m_{a}^{\prime} v_{a}^{\prime}\right\}$, and spins $\left\{\Sigma_{a}^{\prime}\right\}$ at the asymptotic past for $a=1,2, \ldots, M$ and the outgoing particles have masses $\left\{m_{a}\right\}$, velocities $\left\{v_{a}\right\}$, momenta $\left\{p_{a}=m_{a} v_{a}\right\}$, and spins $\left\{\Sigma_{a}\right\}$ at the asymptotic future for $a=1,2, \ldots, N$. Let $X_{a}^{\prime}(\sigma)$ denote the trajectory of the incoming particles in the affine parameter range $-\infty<$ $\sigma \leq 0$ for $a=1,2, \ldots, M$ and $X_{a}(\sigma)$ denotes the trajectory of the outgoing particles in the affine parameter range $0 \leq$ $\sigma<\infty$ for $a=1,2, \ldots, N$. Now to treat incoming and outgoing particles uniformly, we treat the incoming particles as extra outgoing particles under the following identifications:

$$
m_{N+a}=m_{a}^{\prime}, \quad v_{N+a}^{\mu}=-v_{a}^{\prime \mu}, \quad p_{N+a}^{\mu}=-p_{a}^{\prime \mu}, \quad \Sigma_{N+a}^{\mu \nu}=-\Sigma_{a}^{\prime \mu \nu}, \quad X_{N+a}^{\mu}(\sigma)=X_{a}^{\prime \mu}(-\sigma)
$$

for $a=1,2, \ldots, M$ and $0 \leq \sigma<\infty$.

The trajectories and spins of the scattered objects satisfy the following boundary conditions:

$$
\begin{gathered}
X_{a}^{\mu}(\sigma=0)=r_{a}^{\mu},\left.\quad \frac{d X_{a}^{\mu}(\sigma)}{d \sigma}\right|_{\sigma \rightarrow \infty}=v_{a}^{\mu}, \\
\text { and }\left.\quad \Sigma_{a}^{\mu \nu}(\sigma)\right|_{\sigma \rightarrow \infty}=\Sigma_{a}^{\mu \nu} \quad \text { for } a=1,2, \ldots, M+N,
\end{gathered}
$$

with $r_{a}$ being the coordinate on the boundary of region $\mathcal{R}$, where the trajectory of the $a$ th particle intersects. Now outside the region $\mathcal{R}$, the movement of the scattered objects can be well captured by the following matter energy-momentum tensor $[21-32],{ }^{2}$

$$
\begin{aligned}
T^{X \alpha \beta}(x)= & \sum_{a=1}^{M+N} \int_{0}^{\infty} d \sigma\left[m_{a} \frac{d X_{a}^{\alpha}(\sigma)}{d \sigma} \frac{d X_{a}^{\beta}(\sigma)}{d \sigma} \delta^{(4)}\left(x-X_{a}(\sigma)\right)\right] \\
& +\sum_{a=1}^{M+N} \sqrt{-\operatorname{det} g(x)} \nabla_{\gamma}\left[\int_{0}^{\infty} d \sigma \frac{d X_{a}^{(\alpha}(\sigma)}{d \sigma} \Sigma_{a}^{\beta) \gamma}(\sigma) \frac{\delta^{(4)}\left(x-X_{a}(\sigma)\right)}{\sqrt{-\operatorname{det} g(x)}}\right]+\cdots,
\end{aligned}
$$

${ }^{2}$ We are using the following definition of the energy-momentum tensor for the world-line action $S_{X}$ :

$$
T^{X \alpha \beta}(x)=2 \frac{\delta S_{X}}{\delta g_{\alpha \beta}(x)},
$$

which differs from the canonical definition of the energy-momentum tensor by a multiplicative factor of $\sqrt{-\operatorname{det} g}$. 
where unspecified "..." terms contain two or more covariant derivatives operating on the $\sigma$ integral containing the delta function and carry the information of the internal structures of the macroscopic objects in terms of gravitational multipole moments and tidal responses, which will not affect our result to the order we are working in. The symmetrization convention used above is defined as $A^{(\alpha} B^{\beta)}=\frac{1}{2}\left(A^{\alpha} B^{\beta}+A^{\beta} B^{\alpha}\right)$. Now using the definition of a covariant derivative on a rank-3 tensor and the property $\Gamma_{\gamma \delta}^{\gamma}=\frac{1}{\sqrt{-\operatorname{det} g}} \partial_{\delta}(\sqrt{-\operatorname{det} g})$, the above matter energymomentum tensor simplifies to

$$
\begin{aligned}
T^{X \alpha \beta}(x)= & \sum_{a=1}^{M+N} \int_{0}^{\infty} d \sigma\left[m_{a} \frac{d X_{a}^{\alpha}(\sigma)}{d \sigma} \frac{d X_{a}^{\beta}(\sigma)}{d \sigma} \delta^{(4)}\left(x-X_{a}(\sigma)\right)\right. \\
& +\frac{d X_{a}^{(\alpha}(\sigma)}{d \sigma} \Sigma_{a}^{\beta) \gamma}(\sigma) \partial_{\gamma} \delta^{(4)}\left(x-X_{a}(\sigma)\right) \\
& \left.+\Gamma_{\gamma \delta}^{(\alpha}\left(X_{a}(\sigma)\right) \frac{d X_{a}^{\delta}(\sigma)}{d \sigma} \Sigma_{a}^{\beta) \gamma}(\sigma) \delta^{(4)}\left(x-X_{a}(\sigma)\right)\right] .
\end{aligned}
$$

With the above matter energy-momentum tensor, we have to solve the Einstein equation to derive the background metric,

$$
\sqrt{-\operatorname{det} g}\left(R^{\alpha \beta}-\frac{1}{2} R g^{\alpha \beta}\right)=8 \pi G T^{X \alpha \beta} .
$$

We also have to solve the geodesic equation and the equation for the time evolution of spin as given below [28,29,31,33-36]:

$$
\begin{gathered}
\frac{d^{2} X_{a}^{\alpha}(\sigma)}{d \sigma^{2}}+\Gamma_{\beta \gamma}^{\alpha}\left(X_{a}(\sigma)\right) \frac{d X_{a}^{\beta}(\sigma)}{d \sigma} \frac{d X_{a}^{\gamma}(\sigma)}{d \sigma} \\
=-\frac{1}{2 m_{a}} R_{\nu \rho \sigma}^{\alpha}\left(X_{a}(\sigma)\right) \Sigma_{a}^{\rho \sigma}(\sigma) \frac{d X_{a}^{\nu}(\sigma)}{d \sigma}, \\
\frac{d \Sigma_{a}^{\mu \nu}(\sigma)}{d \sigma}+\Gamma_{\alpha \beta}^{\mu}\left(X_{a}(\sigma)\right) \Sigma^{\alpha \nu}(\sigma) \frac{d X_{a}^{\beta}(\sigma)}{d \sigma} \\
+\Gamma_{\alpha \beta}^{\nu}\left(X_{a}(\sigma)\right) \Sigma^{\mu \alpha}(\sigma) \frac{d X_{a}^{\beta}(\sigma)}{d \sigma}=0 .
\end{gathered}
$$

The above equations follow from Mathisson-Papapetrou equations with some correction terms, which are explicitly derived in Appendix A. Also in Appendix A the above equations has been derived demanding the covariant conservation of the canonical version of the matter energymomentum tensor in Eq. (3.10).

Now in terms of the trace reversed metric fluctuation Einstein's equation (3.11) takes the following form:

$$
\eta^{\rho \sigma} \partial_{\rho} \partial_{\sigma} e^{\alpha \beta}(x)=-8 \pi G\left(T^{X \alpha \beta}(x)+T^{h \alpha \beta}(x)\right) \equiv-8 \pi G T^{\alpha \beta}(x),
$$

where $T^{h \alpha \beta}(x)$ is the gravitational energy-momentum tensor defined as

$$
T^{h \alpha \beta} \equiv-\frac{1}{8 \pi G}\left[\sqrt{-\operatorname{det} g}\left(R^{\alpha \beta}-\frac{1}{2} R g^{\alpha \beta}\right)+\eta^{\rho \sigma} \partial_{\rho} \partial_{\sigma} e^{\alpha \beta}\right] .
$$

Now we briefly sketch the strategy of our computation:

(i) We solve Eqs. (3.12)-(3.14) iteratively considering gravitational constant $G$ as an iterative parameter, in the post-Minkowskian (PM) sense.

(ii) At zeroth iterative order we set the initial value of the metric fluctuation $e^{\mu \nu}(x)=0$ and consider the scattered objects travel in asymptotic linearized trajectory $X_{a}^{\mu}(\sigma)=r_{a}^{\mu}+v_{a}^{\mu} \sigma$ with constant value of $\operatorname{spin} \Sigma_{a}^{\mu \nu}(\sigma)=\Sigma_{a}^{\mu \nu}$.

(iii) Next we compute the Fourier transform of the matter energy-momentum tensor given in Eq. (3.10) and keep terms up to subleading order in the $\omega$ expansion. We divide the integration region in Eq. (3.4) into two parts: one is the inside of region $\mathcal{R}$ denoted by $|\vec{x}| \leq L$, where we only need to use the conservation of the energy-momentum tensor, and another is the outside of region $\mathcal{R}$ denoted by $|\vec{x}| \geq L$, where we only need to use the linearized trajectory approximation of scattered objects [21].

(iv) Now using Eq. (3.2) we can read off the order $\mathcal{O}(G)$ gravitational waveform up to subleading order in small $\omega$. Here the leading contribution in small $\omega$ expansion contributes to gravitational DC memory after Fourier transformation in the time variable. But the subleading terms in the $\omega$ expansion, which depends on spin, do not contribute to the displacement kind of memory as it is analytic in the $\omega \rightarrow 0$ limit.

(v) In the first iterative order we use the $\mathcal{O}(G)$ metric fluctuation as the background metric and solve geodesic equation (3.12) with boundary condition (3.6) to find the correction to the linearized trajectory. At this order we also need to solve the spin evolution equation (3.13) with boundary condition (3.7).

(vi) Now using this corrected trajectory, spin correction, and order $\mathcal{O}(G)$ background metric we compute the first iterative correction to the matter and gravitational energy-momentum tensor outside region $\mathcal{R}$. ${ }^{3}$ This first iterative correction to the energymomentum tensor contributes to nonanalytic terms as $\omega \rightarrow 0$ at order $\mathcal{O}\left(\omega^{n} \ln \omega\right)$ for $n \geq 0$ along with analytic terms.

\footnotetext{
${ }^{3}$ Since at this order we are only interested in extracting the nonanalytic terms in $\omega$ for $\omega^{-1} \gg L$, the Fourier transform of the energy-momentum tensor inside the region $\mathcal{R}$ does not contribute to nonanalytic terms in $\omega$.
} 
(vii) Using Eq. (3.2) with the corrected energy-momentum tensor we get the order $\mathcal{O}\left(G^{2}\right)$ gravitational waveform. At this order the leading nonanalytic contribution in small $\omega$ expansion behaves as $\ln \omega$, and this has been evaluated in [13], which contributes to $u^{-1}$ tail memory. So in this article our main goal is to systematically evaluate the order $\omega \ln \omega$ contribution to the gravitational waveform which turns out to be spin dependent and after Fourier transform contributes to $u^{-2}$ tail memory as the first time pointed out in [14].

(viii) Now if we do the analysis in the next iterative order, i.e., at order $\mathcal{O}\left(G^{3}\right)$ of the gravitational waveform, we see that it corrects the order $\omega \ln \omega$ gravitational waveform [14]. Possibly there might be corrections also from order $\mathcal{O}\left(G^{n}\right)$ for $n \geq 4$ to the order $\omega \ln \omega$ gravitational waveform, which seems to be absent by naive dimensional analysis (e.g., see table in Fig. 2). Hence in our analysis we are only able to derive the order $\omega \ln \omega$ contribution of the gravitational waveform at order $\mathcal{O}\left(G^{2}\right)$. So our result should be thought of as the 2PM contribution of order $\omega \ln \omega$ gravitational waveform, which is also equivalent to the 2PM contribution of order $u^{-2}$ gravitational tail memory.

\section{B. Order $\mathcal{O}(G)$ gravitational waveform}

In this subsection we review the analysis of [21] in four spacetime dimensions, ${ }^{4}$ which is necessary for our analysis of the next subsection. Following the strategy, let $\Delta_{(0)} \hat{T}_{<}^{X}$ denote the contribution of the Fourier transform of Eq. (3.10) inside region $\mathcal{R}$ and $\Delta_{(0)} \hat{T}_{>}^{X}$ denotes the contribution of the Fourier transform of Eq. (3.10) outside region $\mathcal{R}$ at order $\mathcal{O}\left(G^{0}\right)$.

After using integration by parts the Fourier transform of Eq. (3.10) outside region $\mathcal{R}$ becomes

$$
\begin{aligned}
\hat{T}_{>}^{X \alpha \beta}(k) & \sum_{a=1}^{M+N} \int_{0}^{\infty} d \sigma e^{-i k . X_{a}(\sigma)}\left[m_{a} \frac{d X_{a}^{\alpha}(\sigma)}{d \sigma} \frac{d X_{a}^{\beta}(\sigma)}{d \sigma}\right. \\
& \left.+i \frac{d X_{a}^{(\alpha}(\sigma)}{d \sigma} \Sigma_{a}^{\beta) \gamma} k_{\gamma}+\Gamma_{\gamma \delta}^{(\alpha}\left(X_{a}(\sigma)\right) \frac{d X_{a}^{\delta}(\sigma)}{d \sigma} \Sigma_{a}^{\beta) \gamma}(\sigma)\right] \\
& -\sum_{a=1}^{M+N} \int_{0}^{\infty} d \sigma \int d^{4} x \delta(|\vec{x}|-L) \delta^{(4)}\left(x-X_{a}(\sigma)\right) e^{-i k . X_{a}(\sigma)} \\
& \times \frac{d X_{a}^{(\alpha}(\sigma)}{d \sigma} \Sigma_{a}^{\beta) \gamma} \tilde{n}_{\gamma},
\end{aligned}
$$

\footnotetext{
${ }^{4}$ There are some sign differences relative to [21] due to the fact that we are considering the velocities of outgoing particles to be positive, where as there the velocities of outgoing particles were considered to be negative.
}

where $\tilde{n}^{\alpha} \simeq\left(0, \frac{\vec{x}}{|\vec{x}|}\right)$ is the outward unit vector on the boundary $\partial \mathcal{R}$. In the last two lines we get the boundary contribution using $\int_{|x| \geq L} d^{4} x \partial_{\gamma} A^{\alpha \beta \gamma}(x)=$ $-\int d^{4} x \delta(|\vec{x}|-L) \tilde{n}_{\gamma} A^{\alpha \beta \gamma}(x)$. Now to evaluate the boundary contribution (last two lines) of the above expression with trajectory $X_{a}(\sigma)=r_{a}+v_{a} \sigma$, we first do the integration over $x$ using the delta function $\delta^{(4)}\left(x-X_{a}(\sigma)\right)$, which substitutes $x=r_{a}+v_{a} \sigma$ everywhere inside the integrand. Now we do the $\sigma$ integral using the delta function $\delta\left(\left|\vec{r}_{a}+\vec{v}_{a} \sigma\right|-L\right)$, which contributes to $1 /\left(v_{a} \cdot \tilde{n}_{a}\right)$ where $\tilde{n}_{a}^{\alpha}=\left(0, \frac{\vec{r}_{a}}{\left|\vec{r}_{a}\right|}\right)$. There is no modulus sign in the contribution from the delta function as for outgoing particles $v_{a} . \tilde{n}_{a}$ is positive. Hence from Eq. (3.16) we get ${ }^{5}$

$$
\begin{aligned}
\Delta_{(0)} & \hat{T}_{>}^{X \alpha \beta}(k) \\
= & \sum_{a=1}^{M+N} e^{-i k \cdot r_{a}}\left[\frac{m_{a} v_{a}^{\alpha} v_{a}^{\beta}}{i\left(v_{a} \cdot k-i \epsilon\right)}+\frac{v_{a}^{(\alpha} \Sigma_{a}^{\beta) \gamma} k_{\gamma}}{v_{a} \cdot k-i \epsilon}\right] \\
& -\sum_{a=1}^{M+N} e^{-i k \cdot r_{a}} \frac{1}{\tilde{n}_{a} \cdot v_{a}} v_{a}^{(\alpha} \Sigma_{a}^{\beta) \gamma} \tilde{n}_{a \gamma} \\
= & (-i) \sum_{a=1}^{M+N}\left[\frac{p_{a}^{\alpha} p_{a}^{\beta}}{p_{a} \cdot k-i \epsilon}+i \frac{p_{a}^{(\alpha} \Sigma_{a}^{\beta) \gamma} k_{\gamma}}{p_{a} \cdot k-i \epsilon}-i k \cdot r_{a} \frac{p_{a}^{\alpha} p_{a}^{\beta}}{p_{a} \cdot k-i \epsilon}\right] \\
& -\sum_{a=1}^{M+N} \frac{1}{\tilde{n}_{a} \cdot p_{a}} p_{a}^{(\alpha} \Sigma_{a}^{\beta) \gamma} \tilde{n}_{a \gamma}+\mathcal{O}(\omega),
\end{aligned}
$$

where in the last two lines we only kept the terms up to subleading order in the $\omega$ expansion and used $p_{a}=m_{a} v_{a}$. The $i \epsilon$ prescription is fixed demanding the finiteness of $\hat{T}^{X}$ from the $\infty$ range of $\sigma$ integration.

Now to derive the Fourier transform of the matter energy-momentum tensor inside the region $\mathcal{R}$, let us start with

$$
\begin{aligned}
-i k_{\alpha} \hat{T}_{<}^{X \alpha \beta}(k) & =\int_{|x| \leq L} d^{4} x \frac{\partial}{\partial x^{\alpha}}\left[e^{-i k . x}\right] T_{\mathrm{in}}^{X \alpha \beta}(x) \\
& =\int d^{4} x \delta(|\vec{x}|-L) e^{-i k \cdot x} \tilde{n}_{\alpha} T_{\mathrm{in}}^{X \alpha \beta}(x),
\end{aligned}
$$

where $T_{\text {in }}^{X \alpha \beta}(x)$ represent the matter energy-momentum tensor inside region $\mathcal{R}$ which is not the same as Eq. (3.10) in general. To get the second line from the first line above, we use integration by parts and consider the conservation law of the matter energy-momentum tensor $\partial_{\alpha} T_{\text {in }}^{X \alpha \beta}=0$ at linearized order. Since the full contribution is written as just a boundary term on $\partial \mathcal{R}, T_{\text {in }}^{X}$ will match with the expression in Eq. (3.10) at the boundary. So in the

\footnotetext{
${ }^{5}$ Here we ignore the term containing the Christoffel connection inside the square bracket of Eq. (3.16), as it will not contribute at order $\mathcal{O}\left(G^{0}\right)$.
} 
last line above we substitute expression (3.10) in place of $T_{\mathrm{in}}^{X}$ and then do integration by parts to remove the derivative over the delta function. Finally after performing the $x$ integration using the delta function, we get ${ }^{6}$

$$
\begin{aligned}
-i k_{\alpha} \hat{T}_{<}^{X \alpha \beta}(k)= & \sum_{a=1}^{M+N} \int_{0}^{\infty} d \sigma e^{-i k . X_{a}(\sigma)} \tilde{n}_{\alpha}\left[\delta\left(\left|\vec{X}_{a}(\sigma)\right|-L\right) m_{a}\right. \\
& \times \frac{d X_{a}^{\alpha}}{d \sigma} \frac{d X_{a}^{\beta}}{d \sigma}-\tilde{n}_{\gamma} \delta^{\prime}\left(\left|\vec{X}_{a}(\sigma)\right|-L\right) \frac{d X_{a}^{(\alpha}}{d \sigma} \Sigma_{a}^{\beta) \gamma} \\
& \left.+i k_{\gamma} \delta\left(\left|\vec{X}_{a}(\sigma)\right|-L\right) \frac{d X_{a}^{(\alpha}}{d \sigma} \Sigma_{a}^{\beta) \gamma}\right]
\end{aligned}
$$

Now we want to evaluate the above expression using asymptotic linearized trajectory $X_{a}(\sigma)=r_{a}+v_{a} \sigma$. To do that we have to use the following two identities of the delta function:

$$
\begin{aligned}
\delta\left(\left|\vec{r}_{a}+\vec{v}_{a} \sigma\right|-L\right) & =\frac{1}{\tilde{n}_{a} \cdot v_{a}} \delta(\sigma), \\
\delta^{\prime}\left(\left|\vec{r}_{a}+\vec{v}_{a} \sigma\right|-L\right) & =\frac{1}{\left(\tilde{n}_{a} \cdot v_{a}\right)^{2}} \delta^{\prime}(\sigma) .
\end{aligned}
$$

Hence using these properties and performing the $\sigma$ integration we get

$$
\begin{aligned}
-i k_{\alpha} \Delta_{(0)} \hat{T}_{<}^{X \alpha \beta}(k)= & \sum_{a=1}^{M+N} e^{-i k \cdot r_{a}}\left[p_{a}^{\beta}-\frac{i}{2} \frac{k \cdot v_{a}}{\tilde{n}_{a} \cdot v_{a}} \Sigma_{a}^{\beta \gamma} \tilde{n}_{a \gamma}\right. \\
& \left.+\frac{i}{2} \Sigma_{a}^{\beta \gamma} k_{\gamma}+\frac{i}{2} \frac{v_{a}^{\beta}}{\tilde{n}_{a} \cdot v_{a}} \tilde{n}_{a \alpha} \Sigma_{a}^{\alpha \gamma} k_{\gamma}\right] \\
= & \sum_{a=1}^{M+N}\left[-i k \cdot r_{a} p_{a}^{\beta}-\frac{i}{2} \frac{k \cdot p_{a}}{\tilde{n}_{a} \cdot p_{a}} \Sigma_{a}^{\beta \gamma} \tilde{n}_{a \gamma}\right. \\
& \left.+\frac{i}{2} \Sigma_{a}^{\beta \gamma} k_{\gamma}+\frac{i}{2} \frac{p_{a}^{\beta}}{\tilde{n}_{a} \cdot p_{a}} \tilde{n}_{a \alpha} \Sigma_{a}^{\alpha \gamma} k_{\gamma}\right] \\
& +\mathcal{O}\left(\omega^{2}\right) .
\end{aligned}
$$

Now from the above expression after stripping out the $k_{\alpha}$ and using total angular momentum conservation relation $\sum_{a}\left(\Sigma_{a}^{\alpha \beta}+r_{a}^{\alpha} p_{a}^{\beta}-r_{a}^{\beta} p_{a}^{\alpha}\right)=0$, we get $^{7}$

$\Delta_{(0)} \hat{T}_{<}^{X \alpha \beta}(k)=\sum_{a=1}^{M+N}\left[r_{a}^{(\alpha} p_{a}^{\beta)}+\frac{p_{a}^{(\alpha}}{\tilde{n}_{a} \cdot p_{a}} \Sigma_{a}^{\beta) \gamma} \tilde{n}_{a \gamma}\right]+\mathcal{O}(\omega)$.

\footnotetext{
${ }^{6}$ Again here we are ignoring the term containing the Christoffel connection as it will not contribute at order $\mathcal{O}\left(G^{0}\right)$ in the analysis of $\hat{T}^{X \alpha \beta}(k)$.

The stripping out of $k_{\alpha}$ is unique up to this order as illustrated in [21].
}

Summing over the contributions of Eqs. (3.17) and (3.22) we find the following order $\mathcal{O}\left(G^{0}\right)$ Fourier transformed energy-momentum tensor:

$$
\begin{aligned}
\Delta_{(0)} \hat{T}^{X \alpha \beta}(k)= & (-i) \sum_{a=1}^{M+N} \frac{1}{\left(p_{a} \cdot k-i \epsilon\right)} \\
& \times\left[p_{a}^{\alpha} p_{a}^{\beta}+i p_{a}^{(\alpha} J_{a}^{\beta) \gamma} k_{\gamma}\right]+\mathcal{O}(\omega),
\end{aligned}
$$

where $J_{a}^{\beta \gamma}=r_{a}^{\beta} p_{a}^{\gamma}-r_{a}^{\gamma} p_{a}^{\beta}+\Sigma_{a}^{\beta \gamma}$ is the total classical angular momentum tensor of particle $a$. Here we can see that neither $r_{a}^{\mu}$ nor $\Sigma_{a}^{\mu \nu}$ are unambiguously defined as there exists transformation $r_{a}^{\mu} \rightarrow r_{a}^{\mu}+c_{a}^{\mu}$ and $\Sigma_{a}^{\mu \nu} \rightarrow \Sigma_{a}^{\mu \nu}-$ $c_{a}^{\mu} p_{a}^{\nu}+c_{a}^{\nu} p_{a}^{\mu}$, under which total angular momentum $J_{a}^{\mu \nu}$ remains invariant. So to give an unambiguous covariant definition of spin angular momentum we are using $p_{a \mu} \Sigma_{a}^{\mu \nu}=0$ from the beginning, which is known as Tulczyjew-Dixon spin supplementary condition [26,37].

Now using the relation (3.2) we get the radiative mode of gravitational waveform at order $\mathcal{O}(G)$ up to subleading order in $\omega$ expansion,

$$
\begin{aligned}
\Delta_{(0)} \tilde{e}^{\mu \nu}(\omega, R, \hat{n}) \simeq & -i \frac{2 G}{R} e^{i \omega R} \sum_{a=1}^{M+N} \frac{1}{\left(p_{a} \cdot k-i \epsilon\right)} \\
& \times\left[p_{a}^{\mu} p_{a}^{\nu}+i p_{a}^{(\mu} J_{a}^{\nu) \rho} k_{\rho}\right] .
\end{aligned}
$$

Now performing the Fourier transform in time variable we get

$$
\Delta_{(0)} e^{\mu \nu}(u, R, \hat{n}) \simeq-\frac{2 G}{R} \sum_{a=1}^{M+N}\left[\frac{p_{a}^{\mu} p_{a}^{\nu}}{p_{a} \cdot \mathbf{n}} H(u)-\frac{p_{a}^{(\mu} J_{a}^{\nu) \rho} \mathbf{n}_{\rho}}{p_{a} \cdot \mathbf{n}} \delta(u)\right],
$$

where $u=t-R$ is the retarded time and $H(u)$ is the Heaviside theta function. Above the first term within the square bracket contributes to gravitational DC memory [1$3,5-8,11,13,18,38,39]$. On the other hand, the second term that depends on spin angular momenta does not contribute to displacement gravitational memory as it is localized near $u=0 .{ }^{8}$ Hence to derive the leading spin-dependent memory we need to go to the next iterative order and hope to get a spin-dependent nonanalytic contribution in small $\omega$ from the Fourier transform of the energy-momentum tensor.

\footnotetext{
${ }^{8}$ But this angular momenta dependent term can contribute to a different kind of spin memory induced by radiative angular momentum flux $[18,40,41]$. This order $\mathcal{O}\left(\omega^{0}\right)$ term in the gravitational waveform receives corrections from order $\mathcal{O}\left(G^{n}\right)$ for $n \geq 2$ which turns out to be theory dependent and depends on the structures of scattered objects. So the full (all PM order) contribution of the order $\mathcal{O}\left(\omega^{0}\right)$ gravitational waveform is nonuniversal $[13,14]$.
} 


\section{Order $\mathcal{O}\left(G^{2}\right)$ gravitational waveform}

Because of the asymptotic linearized trajectory of scattered object $b$, the metric fluctuation can be read off from the solution of linearized Einstein equation (3.11) with the momentum space energy-momentum tensor (3.23),

$$
\begin{aligned}
h_{\alpha \beta}^{(b)}(x)= & -(8 \pi G) \int \frac{d^{4} \ell}{(2 \pi)^{4}} e^{i \ell \cdot x} G_{r}(\ell) \frac{1}{i\left(p_{b} \cdot \ell-i \epsilon\right)}\left[p_{b \alpha} p_{b \beta}-\frac{1}{2} p_{b}^{2} \eta_{\alpha \beta}\right. \\
& \left.+i p_{b(\alpha} J_{b, \beta) \gamma} \ell^{\gamma}-\frac{i}{2} p_{b}^{\delta} J_{b, \delta \gamma} \ell \gamma \eta_{\alpha \beta}\right]
\end{aligned}
$$

where $G_{r}(\ell)$ is the momentum space retarded Green's function given as $G_{r}(\ell)=\left\{\left(\ell^{0}+i \epsilon\right)^{2}-\vec{\ell}^{2}\right\}^{-1}$. We should denote the above metric fluctuation as $\Delta_{(0)} h_{\alpha \beta}^{(b)}$ following our convention, but just to reduce notational complexity we remove the $\Delta_{(0)}$ piece. The Christoffel connection for the metric above is given by

$$
\begin{aligned}
\Gamma_{\nu \rho}^{(b) \mu}(x)= & -(8 \pi G) \int \frac{d^{4} \ell}{(2 \pi)^{4}} e^{i \ell \cdot x} G_{r}(\ell) \frac{1}{p_{b} \cdot \ell-i \epsilon}\left[\ell_{\nu}\left(p_{b \rho} p_{b}^{\mu}-\frac{1}{2} \delta_{\rho}^{\mu} p_{b}^{2}\right)+\ell_{\rho}\left(p_{b \nu} p_{b}^{\mu}-\frac{1}{2} \delta_{\nu}^{\mu} p_{b}^{2}\right)\right. \\
& \left.-\ell^{\mu}\left(p_{b \nu} p_{b \rho}-\frac{1}{2} p_{b}^{2} \eta_{\nu \rho}\right)\right] \\
& -i(8 \pi G) \int \frac{d^{4} \ell}{(2 \pi)^{4}} e^{i \ell \cdot x} G_{r}(\ell) \frac{1}{p_{b} \cdot \ell-i \epsilon}\left[\ell_{\nu} p_{b(\rho} J_{b}^{\mu) \alpha} \ell_{\alpha}+\ell_{\rho} p_{b(\nu} J_{b}^{\mu) \alpha} \ell_{\alpha}-\ell^{\mu} p_{b(\nu} J_{b, \rho) \alpha} \ell^{\alpha}\right. \\
& \left.-\frac{1}{2}\left(\ell_{\nu} \delta_{\rho}^{\mu}+\ell_{\rho} \delta_{\nu}^{\mu}-\ell^{\mu} \eta_{\nu \rho}\right) p_{b}^{\delta} J_{b, \delta \gamma} \ell^{\gamma}\right] .
\end{aligned}
$$

Now due to the nonflat background metric, consider the leading correction to the asymptotic straight-line trajectory of particle $a$ outside the region $\mathcal{R}$ to be

$$
X_{a}^{\mu}(\sigma)=r_{a}^{\mu}+v_{a}^{\mu} \sigma+Y_{a}^{\mu}(\sigma)
$$

with boundary conditions

$$
\left.Y_{a}^{\mu}(\sigma)\right|_{\sigma=0}=0, \quad \text { and }\left.\quad \frac{d Y_{a}^{\mu}(\sigma)}{d \sigma}\right|_{\sigma \rightarrow \infty}=0
$$

Similarly due to the nonflat background metric, there will also be a correction of spin outside the region $\mathcal{R}$, which is given by

$$
\Sigma_{a}^{\mu \nu}(\sigma)=\Sigma_{a}^{\mu \nu}+S_{a}^{\mu \nu}(\sigma)
$$

with boundary condition

$$
S_{a}^{\mu \nu}(\sigma)=0, \quad \text { for }|\sigma| \rightarrow \infty .
$$

Now the order $\mathcal{O}(G)$ contribution of $Y_{a}^{\mu}(\sigma)$ and $S_{a}^{\mu \nu}(\sigma)$ satisfies the equations below, which follow from Eqs. (3.12) and (3.13).

$$
\begin{aligned}
& \frac{d^{2} Y_{a}^{\mu}(\sigma)}{d \sigma^{2}}=-\Gamma_{\nu \rho}^{\mu}\left(r_{a}+v_{a} \sigma\right) v_{a}^{\nu} v_{a}^{\rho}-\frac{1}{2 m_{a}}\left(\partial_{\rho} \Gamma_{\nu \sigma}^{\mu}\left(r_{a}+v_{a} \sigma\right)-\partial_{\sigma} \Gamma_{\nu \rho}^{\mu}\left(r_{a}+v_{a} \sigma\right)\right) \Sigma_{a}^{\rho \sigma} v_{a}^{\nu} \\
& \frac{d S_{a}^{\mu \nu}(\sigma)}{d \sigma}=-\Gamma_{\sigma \rho}^{\mu}\left(r_{a}+v_{a} \sigma\right) \Sigma_{a}^{\rho \nu} v_{a}^{\sigma}+\Gamma_{\sigma \rho}^{\nu}\left(r_{a}+v_{a} \sigma\right) \Sigma_{a}^{\rho \mu} v_{a}^{\sigma}
\end{aligned}
$$

where $\Gamma_{\nu \rho}^{\mu}\left(r_{a}+v_{a} \sigma\right)=\sum_{\substack{b=1 \\ b \neq a}}^{M+N} \Gamma_{\nu \rho}^{(b) \mu}\left(r_{a}+v_{a} \sigma\right)$. After doing integration with the specified boundary conditions of Eqs. (3.29) and (3.31), we get 


$$
\begin{gathered}
\frac{d Y_{a}^{\mu}(\sigma)}{d \sigma}=\int_{\sigma}^{\infty} d \sigma^{\prime}\left[\Gamma_{\nu \rho}^{\mu}\left(r_{a}+v_{a} \sigma^{\prime}\right) v_{a}^{\nu} v_{a}^{\rho}+\frac{1}{2 m_{a}}\left(\partial_{\rho} \Gamma_{\nu \sigma}^{\mu}\left(r_{a}+v_{a} \sigma^{\prime}\right)-\partial_{\sigma} \Gamma_{\nu \rho}^{\mu}\left(r_{a}+v_{a} \sigma^{\prime}\right)\right) \Sigma_{a}^{\rho \sigma} v_{a}^{\nu}\right], \\
S_{a}^{\mu \nu}(\sigma)=\int_{\sigma}^{\infty} d \sigma^{\prime}\left[\Gamma_{\sigma \rho}^{\mu}\left(r_{a}+v_{a} \sigma^{\prime}\right) \Sigma_{a}^{\rho \nu} v_{a}^{\sigma}-\Gamma_{\sigma \rho}^{\nu}\left(r_{a}+v_{a} \sigma^{\prime}\right) \Sigma_{a}^{\rho \mu} v_{a}^{\sigma}\right] .
\end{gathered}
$$

\section{Analysis of matter energy-momentum tensor}

In this section we compute the order $\mathcal{O}(G)$ correction of the Fourier transformation of the matter energy-momentum tensor due to the corrected trajectory and spin of the particle. Since only the nonanalytic terms in $\omega$ contribute to gravitational memory, it is sufficient to analyze the Fourier transformation of the matter energy-momentum tensor outside region $\mathcal{R}$ without the boundary contribution as given in Eq. (3.16). Contributions from the boundary terms and inside the region $\mathcal{R}$ always reproduce terms, which are analytic in $\omega$. Hence neglecting the last two lines of Eq. (3.16) and substituting the corrected trajectory and spin we get

$$
\begin{aligned}
\hat{T}_{>}^{X \mu \nu}(k) \simeq & \sum_{a=1}^{M+N} \int_{0}^{\infty} d \sigma e^{-i k .\left(v_{a} \sigma+r_{a}\right)}\left\{1-i k \cdot Y_{a}(\sigma)+\cdots\right\}\left[m_{a}\left(v_{a}^{\mu} v_{a}^{\nu}+v_{a}^{\mu} \frac{d Y_{a}^{\nu}(\sigma)}{d \sigma}+v_{a}^{\nu} \frac{d Y_{a}^{\mu}(\sigma)}{d \sigma}\right)\right. \\
& \left.+i\left(v_{a}^{(\mu} \Sigma_{a}^{\nu) \alpha}+v_{a}^{(\mu} S_{a}^{\nu) \alpha}(\sigma)+\frac{d Y_{a}^{(\mu}(\sigma)}{d \sigma} \Sigma_{a}^{\nu) \alpha}\right) k_{\alpha}+\Gamma_{\alpha \beta}^{(\mu}\left(r_{a}+v_{a} \sigma\right) \Sigma_{a}^{\nu) \alpha} v_{a}^{\beta}\right] .
\end{aligned}
$$

The order $\mathcal{O}(G)$ correction to the matter energy-momentum tensor

$$
\begin{aligned}
\Delta_{(1)} \hat{T}^{X \mu \nu}(k)= & \sum_{a=1}^{M+N} \int_{0}^{\infty} d \sigma e^{-i k \cdot\left(v_{a} \sigma+r_{a}\right)}\left[-i k . Y_{a}(\sigma) m_{a} v_{a}^{\mu} v_{a}^{\nu}+m_{a} v_{a}^{\mu} \frac{d Y_{a}^{\nu}(\sigma)}{d \sigma}\right. \\
& \left.+m_{a} v_{a}^{\nu} \frac{d Y_{a}^{\mu}(\sigma)}{d \sigma}+i \frac{d Y_{a}^{(\mu}(\sigma)}{d \sigma} \Sigma_{a}^{\nu) \alpha} k_{\alpha}+k \cdot Y_{a}(\sigma) v_{a}^{(\mu} \Sigma_{a}^{\nu) \alpha} k_{\alpha}\right] \\
& +\sum_{a=1}^{M+N} \int_{0}^{\infty} d \sigma e^{-i k \cdot\left(r_{a}+v_{a} \sigma\right)}\left[i v_{a}^{(\mu} S_{a}^{\nu) \alpha}(\sigma) k_{\alpha}+\Gamma_{\alpha \beta}^{(\mu}\left(r_{a}+v_{a} \sigma\right) \Sigma_{a}^{\nu) \alpha} v_{a}^{\beta}\right] .
\end{aligned}
$$

Now performing integration by parts and using the boundary conditions of Eq. (3.29) we get

$$
\begin{aligned}
\Delta_{(1)} \hat{T}^{X \mu \nu}(k)= & \sum_{a=1}^{M+N} e^{-i k \cdot r_{a}}\left[-\frac{p_{a}^{\mu} p_{a}^{\nu}}{p_{a} \cdot k} k_{\beta}+p_{a}^{\mu} \delta_{\beta}^{\nu}+p_{a}^{\nu} \delta_{\beta}^{\mu}+i \delta_{\beta}^{(\mu} \Sigma_{a}^{\nu) \alpha} k_{\alpha}-i \frac{k_{\beta}}{k \cdot p_{a}} p_{a}^{(\mu} \Sigma_{a}^{\nu) \alpha} k_{\alpha}\right] \int_{0}^{\infty} d \sigma e^{-i k \cdot v_{a} \sigma} \frac{d Y_{a}^{\beta}(\sigma)}{d \sigma} \\
& +\sum_{a=1}^{M+N} \int_{0}^{\infty} d \sigma e^{-i k \cdot\left(r_{a}+v_{a} \sigma\right)}\left[i v_{a}^{(\mu} S_{a}^{\nu) \alpha}(\sigma) k_{\alpha}+\Gamma_{\alpha \beta}^{(\mu}\left(r_{a}+v_{a} \sigma\right) \Sigma_{a}^{\nu) \alpha} v_{a}^{\beta}\right] .
\end{aligned}
$$

We evaluate the above integrations using the results of Eqs. (3.33), (3.34), and (3.27) and get

$$
\begin{aligned}
I^{\beta} \equiv & \int_{0}^{\infty} d \sigma e^{-i k \cdot v_{a} \sigma} \frac{d Y_{a}^{\beta}(\sigma)}{d \sigma}=(8 \pi G) \sum_{\substack{b=1 \\
b \neq a}}^{M+N} \int \frac{d^{4} \ell}{(2 \pi)^{4}} e^{i \ell \cdot r_{a}} G_{r}(\ell) \frac{1}{p_{b} \cdot \ell-i \epsilon} \frac{1}{p_{a} \cdot \ell+i \epsilon} \frac{1}{p_{a} \cdot(\ell-k)+i \epsilon} \\
& \times\left[2 p_{a} \cdot \ell\left(p_{a} \cdot p_{b} p_{b}^{\beta}-\frac{1}{2} p_{b}^{2} p_{a}^{\beta}\right)-\ell^{\beta}\left\{\left(p_{a} \cdot p_{b}\right)^{2}-\frac{1}{2} p_{a}^{2} p_{b}^{2}\right\}\right] \\
& +i(8 \pi G) \sum_{\substack{b=1 \\
b \neq a}}^{M+N} \int \frac{d^{4} \ell}{(2 \pi)^{4}} e^{i \ell \cdot r_{a}} G_{r}(\ell) \frac{1}{p_{b} \cdot \ell-i \epsilon} \frac{1}{p_{a} \cdot \ell+i \epsilon} \frac{1}{p_{a} \cdot(\ell-k)+i \epsilon}
\end{aligned}
$$




$$
\begin{aligned}
& \times\left[p_{a} \cdot \ell p_{a} \cdot p_{b} J_{b}^{\beta \alpha} \ell_{\alpha}+p_{a} \cdot \ell p_{b}^{\beta} p_{a \rho} J_{b}^{\rho \alpha} \ell_{\alpha}-\ell^{\beta} p_{a} \cdot p_{b} p_{a \rho} J_{b}^{\rho \alpha} \ell_{\alpha}-\frac{1}{2}\left\{2 p_{a} \cdot \ell p_{a}^{\beta}-\ell^{\beta} p_{a}^{2}\right\} p_{b}^{\delta} J_{b, \delta \gamma} \ell^{\gamma}\right] \\
& +i(8 \pi G) \sum_{\substack{b=1 \\
b \neq a}}^{M+N} \int \frac{d^{4} \ell}{(2 \pi)^{4}} e^{i \ell \cdot r_{a}} G_{r}(\ell) \frac{1}{p_{b} \cdot \ell-i \epsilon} \frac{1}{p_{a} \cdot \ell+i \epsilon} \frac{1}{p_{a} \cdot(\ell-k)+i \epsilon} \\
& \times\left[p_{a} \cdot \ell \ell_{\rho} \Sigma_{a}^{\rho \sigma} p_{b \sigma} p_{b}^{\beta}-\frac{1}{2} p_{b}^{2} p_{a} \cdot \ell \ell_{\rho} \Sigma_{a}^{\rho \beta}-\ell^{\beta} p_{a} \cdot p_{b} \ell_{\rho} \Sigma_{a}^{\rho \sigma} p_{b \sigma}\right]
\end{aligned}
$$

The leading nonanalytic contribution in the small $\omega$ expansion from Eq. (3.37) is of the order of $\ln \omega$ which is determined in [13]. Our goal in this subsection is to extract the $\mathcal{O}(\omega \ln \omega)$ contribution from Eq. (3.37) in the integration region $\omega \ll\left|\ell^{\mu}\right| \ll L^{-1}$. In turn this demands that we have to extract the order $\mathcal{O}(\ln \omega)$ and $\operatorname{order} \mathcal{O}(\omega \ln \omega)$ contributions from the integral expression in Eq. (3.38), which we denote by $I_{1}^{\beta}$ and $I_{2}^{\beta}$, respectively,

$$
\begin{aligned}
I_{1}^{\beta}= & (8 \pi G) \sum_{\substack{b=1 \\
b \neq a}}^{M+N} \int_{\omega}^{L^{-1}} \frac{d^{4} \ell}{(2 \pi)^{4}} G_{r}(\ell) \frac{1}{p_{b} \cdot \ell-i \epsilon} \frac{1}{\left(p_{a} \cdot \ell+i \epsilon\right)^{2}} \\
& \times\left[2 p_{a} \cdot \ell\left(p_{a} \cdot p_{b} p_{b}^{\beta}-\frac{1}{2} p_{b}^{2} p_{a}^{\beta}\right)-\ell^{\beta}\left\{\left(p_{a} \cdot p_{b}\right)^{2}-\frac{1}{2} p_{a}^{2} p_{b}^{2}\right\}\right] \\
= & i \frac{\partial}{\partial p_{a \beta}} K_{g r}^{c l},
\end{aligned}
$$

where [14]

$$
\begin{aligned}
& K_{g r}^{c l}=-\frac{i}{2}(8 \pi G) \sum_{\substack{b, c \\
b \neq c}} \int_{\omega}^{L^{-1}} \frac{d^{4} \ell}{(2 \pi)^{4}} G_{r}(\ell) \frac{1}{p_{b} \cdot \ell+i \epsilon} \frac{1}{p_{c} \cdot \ell-i \epsilon}\left\{\left(p_{b} \cdot p_{c}\right)^{2}-\frac{1}{2} p_{b}^{2} p_{c}^{2}\right\} \\
& =-\frac{i}{2}(2 G) \sum_{\substack{b, c \\
b \neq c \\
\eta_{b} \eta_{c}=1}} \ln \left\{L\left(\omega+i \epsilon \eta_{b}\right)\right\} \frac{\left(p_{b} \cdot p_{c}\right)^{2}-\frac{1}{2} p_{b}^{2} p_{c}^{2}}{\sqrt{\left(p_{b} \cdot p_{c}\right)^{2}-p_{b}^{2} p_{c}^{2}}} . \\
& I_{2}^{\beta}=i(8 \pi G)\left(p_{a} \cdot k\right) \sum_{\substack{b=1 \\
b \neq a}}^{M+N} \int_{\omega}^{L^{-1}} \frac{d^{4} \ell}{(2 \pi)^{4}} \ell \cdot r_{a} G_{r}(\ell) \frac{1}{p_{b} \cdot \ell-i \epsilon} \frac{1}{\left(p_{a} \cdot \ell+i \epsilon\right)^{3}} \\
& \times\left[2 p_{a} \cdot \ell\left(p_{a} \cdot p_{b} p_{b}^{\beta}-\frac{1}{2} p_{b}^{2} p_{a}^{\beta}\right)-\ell^{\beta}\left\{\left(p_{a} \cdot p_{b}\right)^{2}-\frac{1}{2} p_{a}^{2} p_{b}^{2}\right\}\right] \\
& +i(8 \pi G)\left(p_{a} \cdot k\right) \sum_{\substack{b=1 \\
b \neq a}}^{M+N} \int_{\omega}^{L^{-1}} \frac{d^{4} \ell}{(2 \pi)^{4}} G_{r}(\ell) \frac{1}{p_{b} \cdot \ell-i \epsilon} \frac{1}{\left(p_{a} \cdot \ell+i \epsilon\right)^{3}} \\
& \times\left[p_{a} \cdot \ell p_{a} \cdot p_{b} J_{b}^{\beta \alpha} \ell_{\alpha}+p_{a} \cdot \ell p_{b}^{\beta} p_{a \rho} J_{b}^{\rho \alpha} \ell_{\alpha}-\ell^{\beta} p_{a} \cdot p_{b} p_{a \rho} J_{b}^{\rho \alpha} \ell_{\alpha}-\frac{1}{2}\left\{2 p_{a} \cdot \ell p_{a}^{\beta}-\ell^{\beta} p_{a}^{2}\right\} p_{b}^{\delta} J_{b, \delta \gamma} \ell^{\gamma}\right] \\
& +i(8 \pi G) \sum_{\substack{b=1 \\
b \neq a}}^{M+N} \int_{\omega}^{L^{-1}} \frac{d^{4} \ell}{(2 \pi)^{4}} G_{r}(\ell) \frac{1}{p_{b} \cdot \ell-i \epsilon} \frac{1}{\left(p_{a} \cdot \ell+i \epsilon\right)^{3}} p_{a} \cdot k \\
& \times\left[p_{a} \cdot \ell \ell_{\rho} \Sigma_{a}^{\rho \sigma} p_{b \sigma} p_{b}^{\beta}-\frac{1}{2} p_{b}^{2} p_{a} \cdot \ell \ell_{\rho} \Sigma_{a}^{\rho \beta}-\ell^{\beta} p_{a} \cdot p_{b} \ell_{\rho} \Sigma_{a}^{\rho \sigma} p_{b \sigma}\right] .
\end{aligned}
$$

The integral expression of $I_{2}^{\beta}$ above contributes at order $\omega \ln \omega$ and has been explicitly evaluated using contour integration in the complex $\ell^{0}$ plane. The compact form of the result after integration becomes 


$$
\begin{aligned}
I_{2}^{\beta}= & i G\left(p_{a} \cdot k\right) \ln \left\{\left(\omega+i \epsilon \eta_{a}\right) L\right\} \sum_{\substack{b=1 \\
b \neq a \\
\eta_{a} \eta_{b}=1}}^{M+N} \frac{1}{\left[\left(p_{a} \cdot p_{b}\right)^{2}-p_{a}^{2} p_{b}^{2}\right]^{3 / 2}}\left[5\left(p_{a} \cdot p_{b}\right)^{2} p_{b} \cdot r_{a} p_{b}^{\beta}-4 p_{a} \cdot p_{b} p_{b}^{2} p_{a} \cdot r_{a} p_{b}^{\beta}\right. \\
& -2 p_{b}^{2} p_{a} \cdot p_{b} p_{b} \cdot r_{a} p_{a}^{\beta}+2\left(p_{b}^{2}\right)^{2} p_{a} \cdot r_{a} p_{a}^{\beta}-\left(p_{a} \cdot p_{b}\right)^{2} p_{b}^{2} r_{a}^{\beta}-\frac{1}{2} p_{a}^{2} p_{b}^{2} p_{b} \cdot r_{a} p_{b}^{\beta}+\frac{1}{2} p_{a}^{2}\left(p_{b}^{2}\right)^{2} r_{a}^{\beta} \\
& +\left(p_{a} \cdot p_{b}\right)^{2} p_{b}^{2} r_{b}^{\beta}-5\left(p_{a} \cdot p_{b}\right)^{2} p_{b} \cdot r_{b} p_{b}^{\beta}+4 p_{a} \cdot p_{b} p_{b}^{2} p_{a} \cdot r_{b} p_{b}^{\beta}+2 p_{a} \cdot p_{b} p_{b} \cdot r_{b} p_{b}^{2} p_{a}^{\beta}-2\left(p_{b}^{2}\right)^{2} p_{a} \cdot r_{b} p_{a}^{\beta} \\
& \left.+\frac{1}{2} p_{a}^{2} p_{b}^{2} p_{b} \cdot r_{b} p_{b}^{\beta}-\frac{1}{2} p_{a}^{2}\left(p_{b}^{2}\right)^{2} r_{b}^{\beta}-p_{a} \cdot p_{b} p_{b}^{2} \Sigma_{b}^{\beta \alpha} p_{a \alpha}\right] \\
& -3 i G\left(p_{a} \cdot k\right) \ln \left\{\left(\omega+i \epsilon \eta_{a}\right) L\right\} \sum_{\substack{b=1 \\
b \neq a \\
\eta_{a}=1}}^{M+N} \frac{1}{\left[\left(p_{a} \cdot p_{b}\right)^{2}-p_{a}^{2} p_{b}^{2}\right]^{5 / 2}}\left[\left(p_{a} \cdot p_{b} p_{b} \cdot r_{a}-p_{b}^{2} p_{a} \cdot r_{a}\right)\right. \\
& \times\left\{\left(p_{a} \cdot p_{b}\right)^{3} p_{b}^{\beta}-\left(p_{a} \cdot p_{b}\right)^{2} p_{b}^{2} p_{a}^{\beta}-\frac{1}{2} p_{a}^{2} p_{b}^{2} p_{a} \cdot p_{b} p_{b}^{\beta}+\frac{1}{2} p_{a}^{2}\left(p_{b}^{2}\right)^{2} p_{a}^{\beta}\right\} \\
& +\left(p_{a} \cdot p_{b}\right)^{3} p_{b}^{2} p_{a} \cdot r_{b} p_{b}^{\beta}+\left(p_{a} \cdot p_{b}\right)^{3} p_{b}^{2} p_{b} \cdot r_{b} p_{a}^{\beta}-\frac{1}{2} p_{a}^{2}\left(p_{b}^{2}\right)^{2} p_{a} \cdot p_{b} p_{a} \cdot r_{b} p_{b}^{\beta}-\frac{1}{2} p_{a}^{2}\left(p_{b}^{2}\right)^{2} p_{a} \cdot p_{b} p_{b} \cdot r_{b} p_{a}^{\beta} \\
& \left.-\left(p_{a} \cdot p_{b}\right)^{4} p_{b} \cdot r_{b} p_{b}^{\beta}+\frac{1}{2}\left(p_{a} \cdot p_{b}\right)^{2} p_{a}^{2} p_{b}^{2} p_{b} \cdot r_{b} p_{b}^{\beta}-\left(p_{a} \cdot p_{b}\right)^{2}\left(p_{b}^{2}\right)^{2} p_{a} \cdot r_{b} p_{a}^{\beta}+\frac{1}{2} p_{a}^{2}\left(p_{b}^{2}\right)^{3} p_{a} \cdot r_{b} p_{a}^{\beta}\right] .
\end{aligned}
$$

Above $\eta_{b}=+1$ if particle $b$ is outgoing and $\eta_{b}=-1$ if particle $b$ is ingoing. We also need to evaluate the last line of Eq. (3.37), which contributes at $\operatorname{order} \mathcal{O}(\omega \ln \omega)$ in the integration region $\omega \ll\left|\ell^{\mu}\right| \ll L^{-1}$,

$$
\begin{aligned}
& \sum_{a=1}^{M+N} \int_{0}^{\infty} d \sigma e^{-i k \cdot\left(r_{a}+v_{a} \sigma\right)}\left[i v_{a}^{(\mu} S_{a}^{\nu) \alpha}(\sigma) k_{\alpha}+\Gamma_{\alpha \beta}^{(\mu}\left(r_{a}+v_{a} \sigma\right) \Sigma_{a}^{\nu) \alpha} v_{a}^{\beta}\right] \\
& \simeq+i G \sum_{a=1}^{M+N} \sum_{\substack{b=1 \\
b \neq a \\
\eta_{a} \eta_{b}=1}}^{M+N} \ln \left\{\left(\omega+i \epsilon \eta_{a}\right) L\right\} \frac{1}{\left[\left(p_{a} \cdot p_{b}\right)^{2}-p_{a}^{2} p_{b}^{2}\right]^{3 / 2}} \\
& \quad \times\left[\left\{\left(p_{a} \cdot p_{b}\right)^{2}-p_{a}^{2} p_{b}^{2}\right\} p_{b \rho} \Sigma_{a}^{\rho \alpha} k_{\alpha}\left(p_{a}^{\mu} p_{b}^{\nu}+p_{a}^{\nu} p_{b}^{\mu}\right)-p_{b}^{2}\left\{\left(p_{a} \cdot p_{b}\right)^{2}-p_{a}^{2} p_{b}^{2}\right\}\left(p_{a}^{\mu} \Sigma_{a}^{\nu \alpha} k_{\alpha}+p_{a}^{\nu} \Sigma_{a}^{\mu \alpha} k_{\alpha}\right)\right. \\
& \quad-\left\{\left(p_{a} \cdot p_{b}\right)^{2}-p_{a}^{2} p_{b}^{2}\right\} p_{b} \cdot k\left(p_{a}^{\mu} p_{b \rho} \Sigma_{a}^{\rho \nu}+p_{a}^{\nu} p_{b \rho} \Sigma_{a}^{\rho \mu}\right)+p_{a} \cdot p_{b} p_{b}^{2} p_{b \rho} \Sigma_{a}^{\rho \alpha} k_{\alpha} p_{a}^{\mu} p_{a}^{\nu} \\
& \left.\quad+\left(p_{a} \cdot k\right)\left\{\left(p_{a} \cdot p_{b}\right)^{2}-p_{a}^{2} p_{b}^{2}\right\}\left(p_{b}^{\mu} p_{b \alpha} \Sigma_{a}^{\alpha \nu}+p_{b}^{\nu} p_{b \alpha} \Sigma_{a}^{\alpha \mu}\right)\right] .
\end{aligned}
$$

Now using these results of integrations, the order $\mathcal{O}(\omega \ln \omega)$ contribution to the matter energy-momentum tensor from Eq. (3.37) becomes

$$
\begin{aligned}
\Delta_{(1)}^{(\omega \ln \omega)} \hat{T}^{X \mu \nu}(k)= & -\sum_{a=1}^{M+N}\left(k \cdot r_{a}\right)\left[\frac{p_{a}^{\mu} p_{a}^{\nu}}{p_{a} \cdot k} k^{\alpha} \frac{\partial}{\partial p_{a}^{\alpha}} K_{g r}^{c l}-\frac{1}{2} p_{a}^{\mu} \frac{\partial}{\partial p_{a \nu}} K_{g r}^{c l}-\frac{1}{2} p_{a}^{\nu} \frac{\partial}{\partial p_{a \mu}} K_{g r}^{c l}\right] \\
& +\frac{1}{2} \sum_{a=1}^{M+N}\left[\frac{1}{p_{a} \cdot k}\left\{p_{a}^{\mu} \Sigma_{a}^{\nu \alpha} k_{\alpha}+p_{a}^{\nu} \Sigma_{a}^{\mu \alpha} k_{\alpha}\right\} k^{\beta} \frac{\partial}{\partial p_{a}^{\beta}} K_{g r}^{c l}-\Sigma_{a}^{\nu \alpha} k_{\alpha} \frac{\partial}{\partial p_{a \mu}} K_{g r}^{c l}-\Sigma_{a}^{\mu \alpha} k_{\alpha} \frac{\partial}{\partial p_{a \nu}} K_{g r}^{c l}\right] \\
& -\sum_{a=1}^{M+N}\left[\frac{p_{a}^{\mu} p_{a}^{\nu}}{p_{a} \cdot k} k_{\beta} I_{2}^{\beta}-p_{a}^{\mu} I_{2}^{\nu}-p_{a}^{\nu} I_{2}^{\mu}\right]+\sum_{a=1}^{M+N}\left(k \cdot r_{a}\right)\left[\frac{1}{2} p_{a}^{\mu} \frac{\partial}{\partial p_{a \nu}} K_{g r}^{c l}+\frac{1}{2} p_{a}^{\nu} \frac{\partial}{\partial p_{a \mu}} K_{g r}^{c l}\right]
\end{aligned}
$$




$$
\begin{aligned}
& +i G \sum_{a=1}^{M+N} \sum_{\substack{b=1 \\
b \neq a \\
\eta^{\prime} \eta_{b}=1}}^{M+N} \ln \left\{\left(\omega+i \epsilon \eta_{a}\right) L\right\} \frac{1}{\left[\left(p_{a} \cdot p_{b}\right)^{2}-p_{a}^{2} p_{b}^{2}\right]^{3 / 2}} \\
& \times\left[\left\{\left(p_{a} \cdot p_{b}\right)^{2}-p_{a}^{2} p_{b}^{2}\right\} p_{b \rho} \Sigma_{a}^{\rho \alpha} k_{\alpha}\left(p_{a}^{\mu} p_{b}^{\nu}+p_{a}^{\nu} p_{b}^{\mu}\right)-p_{b}^{2}\left\{\left(p_{a} \cdot p_{b}\right)^{2}-p_{a}^{2} p_{b}^{2}\right\}\left(p_{a}^{\mu} \Sigma_{a}^{\nu \alpha} k_{\alpha}+p_{a}^{\nu} \Sigma_{a}^{\mu \alpha} k_{\alpha}\right)\right. \\
& -\left\{\left(p_{a} \cdot p_{b}\right)^{2}-p_{a}^{2} p_{b}^{2}\right\} p_{b} \cdot k\left(p_{a}^{\mu} p_{b \rho} \Sigma_{a}^{\rho \nu}+p_{a}^{\nu} p_{b \rho} \Sigma_{a}^{\rho \mu}\right)+p_{a} \cdot p_{b} p_{b}^{2} p_{b \rho} \Sigma_{a}^{\rho \alpha} k_{\alpha} p_{a}^{\mu} p_{a}^{\nu} \\
& \left.+\left(p_{a} \cdot k\right)\left\{\left(p_{a} \cdot p_{b}\right)^{2}-p_{a}^{2} p_{b}^{2}\right\}\left(p_{b}^{\mu} p_{b \alpha} \Sigma_{a}^{\alpha \nu}+p_{b}^{\nu} p_{b \alpha} \Sigma_{a}^{\alpha \mu}\right)\right]
\end{aligned}
$$

\section{Analysis of gravitational energy-momentum tensor}

Here we will compute the spin-dependent gravitational energy-momentum tensor for the metric fluctuation given in Eq. (3.26). Fourier transform of the order $G$ gravitational energy-momentum tensor takes the following form:

$$
\begin{aligned}
\Delta_{(1)} \hat{T}^{h \mu \nu}(k)= & -(8 \pi G) \sum_{a, b=1}^{M+N} \int \frac{d^{4} \ell}{(2 \pi)^{4}} G_{r}(k-\ell) G_{r}(\ell) \frac{1}{p_{b} \cdot \ell-i \epsilon} \frac{1}{p_{a} \cdot(k-\ell)-i \epsilon} \\
& \times\left\{p_{b \alpha} p_{b \beta}-\frac{1}{2} p_{b}^{2} \eta_{\alpha \beta}+i p_{b(\alpha} J_{b, \beta) \gamma} \ell^{\gamma}-\frac{i}{2} \eta_{\alpha \beta} p_{b}^{\delta} J_{b, \delta \gamma} \ell^{\gamma}\right\} \mathcal{F}^{\mu \nu, \alpha \beta, \rho \sigma}(k, \ell) \\
& \times\left\{p_{a \rho} p_{a \sigma}-\frac{1}{2} p_{a}^{2} \eta_{\rho \sigma}+i p_{a(\rho} J_{a, \sigma) \delta}(k-\ell)^{\delta}-\frac{i}{2} \eta_{\rho \sigma} p_{a}^{\kappa} J_{a, \kappa \tau}(k-\ell)^{\tau}\right\},
\end{aligned}
$$

where $[13,14]$

$$
\begin{aligned}
\mathcal{F}^{\mu \nu, \alpha \beta, \rho \sigma}(k, \ell)= & 2\left[\frac{1}{2} \ell^{\mu}(k-\ell)^{\nu} \eta^{\alpha \rho} \eta^{\beta \sigma}+(k-\ell)^{\mu}(k-\ell)^{\nu} \eta^{\alpha \rho} \eta^{\beta \sigma}-(k-\ell)^{\nu}(k-\ell)^{\beta} \eta^{\alpha \rho} \eta^{\mu \sigma}\right. \\
& -(k-\ell)^{\mu}(k-\ell)^{\beta} \eta^{\alpha \rho} \eta^{\nu \sigma}+(k-\ell)^{\alpha}(k-\ell)^{\beta} \eta^{\mu \rho} \eta^{\nu \sigma}+(k-\ell) \cdot \ell \eta^{\nu \beta} \eta^{\alpha \rho} \eta^{\mu \sigma} \\
& \left.-\ell^{\rho}(k-\ell)^{\alpha} \eta^{\nu \beta} \eta^{\mu \sigma}-\frac{1}{2}(k-\ell)^{2} \eta^{\mu \alpha} \eta^{\nu \beta} \eta^{\rho \sigma}+\eta^{\mu \alpha} \eta^{\beta \rho} \eta^{\nu \sigma}(k-\ell)^{2}+\eta^{\nu \alpha} \eta^{\beta \rho} \eta^{\mu \sigma}(k-\ell)^{2}\right] \\
& -\eta^{\mu \nu}\left[\frac{3}{2}(k-\ell) \cdot \ell \eta^{\alpha \rho} \eta^{\beta \sigma}+2(k-\ell)^{2} \eta^{\alpha \rho} \eta^{\beta \sigma}-\ell^{\sigma}(k-\ell)^{\alpha} \eta^{\beta \rho}\right] \\
& -\eta^{\alpha \beta}(k-\ell)^{2} \eta^{\mu \rho} \eta^{\nu \sigma}+\frac{1}{2} \eta^{\alpha \beta}(k-\ell)^{2} \eta^{\rho \sigma} \eta^{\mu \nu}
\end{aligned}
$$

follows from the quadratic part of the gravitational energy-momentum tensor defined in Eq. (3.15). In the integration region $R^{-1} \ll\left|\ell^{\mu}\right| \ll \omega$, at leading order $\mathcal{F}(k, \ell)$ approximates in the following form up to gauge equivalence:

$$
\mathcal{F}^{\mu \nu, \alpha \beta, \rho \sigma}(k, \ell) \simeq-2 k^{\beta} k^{\sigma} \eta^{\alpha \rho} \eta^{\mu \nu}+2 k^{\alpha} k^{\beta} \eta^{\mu \rho} \eta^{\nu \sigma} .
$$

Hence in the integration region $R^{-1} \ll\left|\ell^{\mu}\right| \ll \omega$ the order $\mathcal{O}(\omega \ln \omega)$ contribution from Eq. (3.45) turns out to be

$$
\begin{aligned}
\Delta_{(1)}^{(\omega \ln \omega)} \hat{T}_{1}^{h \mu \nu}(k)= & -(8 \pi G) \sum_{a, b=1}^{M+N} \frac{1}{p_{a} \cdot k}\left\{p_{b \alpha} p_{b \beta}-\frac{1}{2} p_{b}^{2} \eta_{\alpha \beta}\right\}\left[-2 k^{\beta} k^{\sigma} \eta^{\alpha \rho} \eta^{\mu \nu}+2 k^{\alpha} k^{\beta} \eta^{\mu \rho} \eta^{\nu \sigma}\right] \\
& \times\left\{i p_{a(\rho} J_{a, \sigma) \delta} k^{\delta}-\frac{i}{2} \eta_{\rho \sigma} p_{a}^{\kappa} J_{a, k \tau} k^{\tau}\right\} \int_{R^{-1}}^{\omega} \frac{d^{4} \ell}{(2 \pi)^{4}} G_{r}(\ell) \frac{1}{p_{b} \cdot \ell-i \epsilon} \frac{1}{2 k \cdot \ell+i \epsilon} .
\end{aligned}
$$

Now after using the result of the integration [13]

$$
\int_{R^{-1}}^{\omega} \frac{d^{4} \ell}{(2 \pi)^{4}} G_{r}(\ell) \frac{1}{p_{b} \cdot \ell-i \epsilon} \frac{1}{k \cdot \ell+i \epsilon}=\frac{1}{4 \pi} \delta_{\eta_{b}, 1} \frac{1}{p_{b} \cdot k} \ln \{(\omega+i \epsilon) R\},
$$

we get 


$$
\Delta_{(1)}^{(\omega \ln \omega)} \hat{T}_{1}^{h \mu \nu}(k)=-i G \ln \{(\omega+i \epsilon) R\} \sum_{b=1}^{N} \sum_{a=1}^{M+N}\left[-\eta^{\mu \nu} p_{b}^{\rho} J_{a, \rho \sigma} k^{\sigma}+\frac{p_{b} \cdot k}{p_{a} \cdot k}\left(p_{a}^{\mu} J_{a}^{\nu \rho} k_{\rho}+p_{a}^{\nu} J_{a}^{\mu \rho} k_{\rho}\right)\right]
$$

After using the total angular momentum conservation relation $\sum_{a=1}^{M+N} J_{a}^{\rho \sigma}=0$, we get

$$
\Delta_{(1)}^{(\omega \ln \omega)} \hat{T}_{1}^{h \mu \nu}(k)=-2 i G \ln \{(\omega+i \epsilon) R\} \sum_{b=1}^{N} p_{b} \cdot k \sum_{a=1}^{M+N} \frac{1}{p_{a} \cdot k} p_{a}^{(\mu} J_{a}^{\nu) \alpha} k_{\alpha} .
$$

Next we analyze the expression in Eq. (3.45) in the integration region $\omega \ll\left|\ell^{\mu}\right| \ll L^{-1}$ and extract the order $\mathcal{O}(\omega \ln \omega)$ contribution. First, let us substitute the following identity in Eq. (3.45):

$$
G_{r}(k-\ell) G_{r}(\ell)=G_{r}(k-\ell) G_{r}(-\ell)-2 \pi i \delta\left(\ell^{2}\right)\left[H\left(\ell^{0}\right)-H\left(-\ell^{0}\right)\right] G_{r}(k-\ell),
$$

and focus on the contribution coming from the $G_{r}(k-\ell) G_{r}(-\ell)$ part. As described in Appendix C, the gravitational energy-momentum tensor with the $\delta\left(\ell^{2}\right)\left[H\left(\ell^{0}\right)-H\left(-\ell^{0}\right)\right] G_{r}(k-\ell)$ part does not contribute to order $\mathcal{O}(\omega \ln \omega)$ in the integration region $\omega \ll\left|\ell^{\mu}\right| \ll L^{-1}$. Following the discussion of Appendix B in [13], a part of this contribution can be identified with the soft radiation from the finite energy real gravitational radiation, which is already taken care of in the hard particle sums of the earlier expressions. So in the integration region $\omega \ll\left|\ell^{\mu}\right| \ll L^{-1}$, the order $\mathcal{O}(\omega \ln \omega)$ contribution from the gravitational energy-momentum tensor with the $G_{r}(k-\ell) G_{r}(-\ell)$ part turns out to be

$$
\begin{aligned}
& \Delta_{(1)}^{(\omega \ln \omega)} \hat{T}_{2}^{h \mu \nu}(k)=(8 \pi G) \sum_{a, b=1}^{M+N} \int_{\omega}^{L^{-1}} \frac{d^{4} \ell}{(2 \pi)^{4}}\left\{G_{r}(-\ell)\right\}^{3}(-2 k . \ell) \frac{1}{p_{b} \cdot \ell-i \epsilon} \frac{1}{p_{a} . \ell+i \epsilon} \\
& \times\left[\left\{i p_{b}^{(\alpha} J_{b}^{\beta) \gamma} \ell_{\gamma}-\frac{i}{2} \eta^{\alpha \beta} p_{b \kappa} J_{b}^{\kappa \gamma} \ell_{\gamma}\right\} \Delta_{(\ell \ell)} \mathcal{F}^{\mu \nu}{ }_{\alpha \beta, \rho \sigma}(k, \ell)\left\{p_{a}^{\rho} p_{a}^{\sigma}-\frac{1}{2} p_{a}^{2} \eta^{\rho \sigma}\right\}\right. \\
& \left.+\left\{p_{b}^{\alpha} p_{b}^{\beta}-\frac{1}{2} p_{b}^{2} \eta^{\alpha \beta}\right\} \Delta_{(\ell \ell)} \mathcal{F}^{\mu \nu}{ }_{\alpha \beta, \rho \sigma}(k, \ell)\left\{-i p_{a}^{(\rho} J_{a}^{\sigma) \delta} \ell_{\delta}+\frac{i}{2} \eta^{\rho \sigma} p_{a \kappa} J_{a}^{\kappa \delta} \ell_{\delta}\right\}\right] \\
& +(8 \pi G) \sum_{a, b=1}^{M+N} \int_{\omega}^{L^{-1}} \frac{d^{4} \ell}{(2 \pi)^{4}}\left\{G_{r}(-\ell)\right\}^{2} \frac{1}{p_{b} \cdot \ell-i \epsilon} \frac{1}{\left(p_{a} \cdot \ell+i \epsilon\right)^{2}}\left(p_{a} \cdot k\right) \\
& \times\left[\left\{i p_{b}^{(\alpha} J_{b}^{\beta) \gamma} \ell_{\gamma}-\frac{i}{2} \eta^{\alpha \beta} p_{b \kappa} J_{b}^{\kappa \gamma} \ell_{\gamma}\right\} \Delta_{(\ell \ell)} \mathcal{F}^{\mu \nu}{ }_{\alpha \beta, \rho \sigma}(k, \ell)\left\{p_{a}^{\rho} p_{a}^{\sigma}-\frac{1}{2} p_{a}^{2} \eta^{\rho \sigma}\right\}\right. \\
& \left.+\left\{p_{b}^{\alpha} p_{b}^{\beta}-\frac{1}{2} p_{b}^{2} \eta^{\alpha \beta}\right\} \Delta_{(\ell \ell)} \mathcal{F}^{\mu \nu}{ }_{\alpha \beta, \rho \sigma}(k, \ell)\left\{-i p_{a}^{(\rho} J_{a}^{\sigma) \delta} \ell_{\delta}+\frac{i}{2} \eta^{\rho \sigma} p_{a \kappa} J_{a}^{\kappa \delta} \ell_{\delta}\right\}\right] \\
& +(8 \pi G) \sum_{a, b=1}^{M+N} \int_{\omega}^{L^{-1}} \frac{d^{4} \ell}{(2 \pi)^{4}}\left\{G_{r}(-\ell)\right\}^{2} \frac{1}{p_{b} \cdot \ell-i \epsilon} \frac{1}{p_{a} \cdot \ell+i \epsilon} \\
& \times\left[\left\{i p_{b}^{(\alpha} J_{b}^{\beta) \gamma} \ell_{\gamma}-\frac{i}{2} \eta^{\alpha \beta} p_{b \kappa} J_{b}^{\kappa \gamma} \ell_{\gamma}\right\} \Delta_{(k \ell)} \mathcal{F}^{\mu \nu}{ }_{\alpha \beta, \rho \sigma}(k, \ell)\left\{p_{a}^{\rho} p_{a}^{\sigma}-\frac{1}{2} p_{a}^{2} \eta^{\rho \sigma}\right\}\right. \\
& \left.+\left\{p_{b}^{\alpha} p_{b}^{\beta}-\frac{1}{2} p_{b}^{2} \eta^{\alpha \beta}\right\} \Delta_{(k \ell)} \mathcal{F}_{\alpha \beta, \rho \sigma}^{\mu \nu}(k, \ell)\left\{-i p_{a}^{(\rho} J_{a}^{\sigma) \delta} \ell_{\delta}+\frac{i}{2} \eta^{\rho \sigma} p_{a \kappa} J_{a}^{\kappa \delta} \ell_{\delta}\right\}\right] \\
& +(8 \pi G) \sum_{a, b=1}^{M+N} \int_{\omega}^{L^{-1}} \frac{d^{4} \ell}{(2 \pi)^{4}}\left\{G_{r}(-\ell)\right\}^{2} \frac{1}{p_{b} \cdot \ell-i \epsilon} \frac{1}{p_{a} \cdot \ell+i \epsilon} \\
& \times\left\{p_{b}^{\alpha} p_{b}^{\beta}-\frac{1}{2} p_{b}^{2} \eta^{\alpha \beta}\right\} \Delta_{(\ell \ell)} \mathcal{F}^{\mu \nu}{ }_{\alpha \beta, \rho \sigma}(k, \ell)\left\{i p_{a}^{(\rho} J_{a}^{\sigma) \delta} k_{\delta}-\frac{i}{2} \eta^{\rho \sigma} p_{a \kappa} J_{a}^{\kappa \delta} k_{\delta}\right\} .
\end{aligned}
$$

In the above expression, $\Delta_{(\ell \ell)} \mathcal{F}(k, \ell)$ and $\Delta_{(k \ell)} \mathcal{F}(k, \ell)$ correspond to the order $\mathcal{O}(\ell \ell)$ and order $\mathcal{O}(k \ell)$ contributions of $\mathcal{F}(k, \ell)$, respectively, which can easily be extractable from Eq. (3.46). The expression in Eq. (3.53) is explicitly evaluated in Appendix B and the final result of the order $\mathcal{O}(\omega \ln \omega)$ contribution is written in Eq. (B18). 


\section{Total energy-momentum tensor and gravitational waveform at order $\mathcal{O}(\omega \ln \omega)$}

Summing over the contributions of Eqs. (3.44), (B18), and (3.51), we get the Fourier transform of the total energymomentum tensor at order $\mathcal{O}(G \omega \ln \omega)$ :

$$
\begin{aligned}
& \Delta_{(1)}^{(\omega \ln \omega)} \hat{T}^{\mu \nu}(k) \\
&=-2 i G \ln \{(\omega+i \epsilon) R\} \sum_{b=1}^{N} p_{b} \cdot k \sum_{a=1}^{M+N} \frac{1}{p_{a} \cdot k} p_{a}^{(\mu} J_{a}^{\nu) \alpha} k_{\alpha} \\
&-\frac{1}{2} \sum_{a=1}^{M+N} \frac{k_{\rho} k_{\sigma}}{p_{a} \cdot k}\left\{\left(p_{a}^{\mu} \frac{\partial}{\partial p_{a \rho}}-p_{a}^{\rho} \frac{\partial}{\partial p_{a \mu}}\right) K_{g r}^{c l}\right. \\
& \times\left(r_{a}^{\sigma} p_{a}^{\nu}-r_{a}^{\nu} p_{a}^{\sigma}+\Sigma_{a}^{\sigma \nu}\right) \\
&\left.+\left(p_{a}^{\nu} \frac{\partial}{\partial p_{a \sigma}}-p_{a}^{\sigma} \frac{\partial}{\partial p_{a \nu}}\right) K_{g r}^{c l} \times\left(r_{a}^{\rho} p_{a}^{\mu}-r_{a}^{\mu} p_{a}^{\rho}+\Sigma_{a}^{\rho \mu}\right)\right\} .
\end{aligned}
$$

Using the relation in Eq. (3.2), the radiative mode of the gravitational waveform at order $\mathcal{O}\left(G^{2} \omega \ln \omega\right)$ for the above derived energy-momentum tensor takes the following form:

$$
\begin{aligned}
& \Delta_{\left(G^{2}\right)}^{(\omega \ln \omega)} \tilde{e}^{\mu \nu}(\omega, \vec{x}) \\
&=\frac{2 G}{R} \exp \{i \omega R\}\left[2 i G \ln \{(\omega+i \epsilon) R\} \sum_{b=1}^{N} p_{b} \cdot k\right. \\
& \quad \times \sum_{a=1}^{M+N} \frac{p_{a}^{(\mu} k_{\rho}}{p_{a} \cdot k}\left(r_{a}^{\rho} p_{a}^{\nu)}-r_{a}^{\nu)} p_{a}^{\rho}+\Sigma_{a}^{\rho \nu)}\right) \\
&-\frac{1}{2} \sum_{a=1}^{M+N} \frac{k_{\rho} k_{\sigma}}{p_{a} \cdot k}\left\{\left(p_{a}^{\mu} \frac{\partial}{\partial p_{a \rho}}-p_{a}^{\rho} \frac{\partial}{\partial p_{a \mu}}\right) K_{g r}^{c l}\right. \\
& \times\left(r_{a}^{\sigma} p_{a}^{\nu}-r_{a}^{\nu} p_{a}^{\sigma}+\Sigma_{a}^{\sigma \nu}\right) \\
&\left.\left.+\left(p_{a}^{\nu} \frac{\partial}{\partial p_{a \sigma}}-p_{a}^{\sigma} \frac{\partial}{\partial p_{a \nu}}\right) K_{g r}^{c l} \times\left(r_{a}^{\rho} p_{a}^{\mu}-r_{a}^{\mu} p_{a}^{\rho}+\Sigma_{a}^{\rho \mu}\right)\right\}\right] .
\end{aligned}
$$

Now if we compare the above expression with the conjectured result given in Eq. (2.7), we observe that our result in the direct derivation completely agrees with the conjectured waveform.

\section{CONCLUSION}

In this paper, we derive the leading spin-dependent gravitational tail memory which behaves as $u^{-2}$ for retarded time $u \rightarrow \pm \infty$. First, we predict the result from the classical limit of the soft graviton theorem, and then we derive it for a general gravitational scattering process involving spinning objects. The final result of leading spin-dependent gravitational tail memory has been summarized in Secs. II $\mathrm{A}$ and II B. Here we are pointing out the novel features of our result, its theoretical and observational importance, and a possible way of rederiving our result with other available prescriptions:

(1) Even when the scattered objects do not carry any intrinsic spins, still a large part of the order $\mathcal{O}\left(G^{2} \omega \ln \omega\right)$ gravitational waveform is nonvanishing and can be read off from Eq. (2.8) by setting $\Sigma_{a}=\Sigma_{a}^{\prime}=0$. This result is fully determined in terms of the asymptotic momenta, asymptotic orbital angular momenta, and the direction cosine and frequency of gravitational wave emission.

(2) Our result of order $u^{-2}$ gravitational memory as given in Eqs. (2.9) and (2.10) has theoretical and observational importance in the current era of gravitational wave physics. First of all, from the observation of this tail memory one can read off the spins of scattered objects, which was not possible for earlier known DC memory or even $u^{-1}$ and $u^{-2} \ln u$ tail memories. Second, for a black hole binary merger process even if the black holes carry spin, still in Sec. II B we have shown that gravitational tail memory at this order vanishes, which is another nontrivial prediction from general relativity. In Sec. II B we also have shown that if the scattering event carries some massless particles or radiation along with the high-frequency gravitational wave in the final state, still the waveform can be rewritten in such a way that it does not carry any information about the outgoing massless particles or radiation.

(3) In the recent past there has been a lot of progress in deriving various classical observables including gravitational radiation for $2 \rightarrow 2$ scattering of spinning bodies under weak gravitational interaction $[22,23,42,43]$. There are two differences between our analysis and the analysis done in the papers cited above. The first one is about setting the boundary conditions: to derive the low-frequency gravitational waveform, we give both initial and final data for the scattered objects. This has the advantage that we do not have to specify the kind of interaction and strength within the region $\mathcal{R}$. On the other hand, in Refs. [22,23,42,43], one specifies only the initial data for the scattered objects and derive various classical observables assuming the weak gravitational interaction responsible for the scattering event. Another difference is that in Refs. [22,23,42,43] the gravitational radiation has been expressed as an integral form without the evaluation of the integrals in the low-frequency limit (soft region), as done here. But recently in [44] an attempt has been taken to relate these two approaches for the nonspinning $2 \rightarrow 2$ particle scattering event under weak gravitational interaction. It is possible to generalize this idea for spinning particle scattering and rederive our result. 


\section{ACKNOWLEDGMENTS}

We are deeply thankful to Alok Laddha, Arnab Priya Saha, Ashoke Sen, and Amitabh Virmani for many enlightening discussions, encouragements, and useful comments on the draft. The work of D. G. is supported by a grant to Chennai Mathematical Institute from the Infosys foundation. The work of B.S. is supported by the Simons Foundation Grant No. 488649 (Simons Collaboration on the Nonperturbative Bootstrap) and by the Swiss National Science Foundation through the National Centre of Competence in Research SwissMAP. B. S. also thanks the organizers and participants of the workshop Gravitational scattering, inspiral, and radiation at the Galileo Galilei Institute for Theoretical Physics in Florence, for hospitality and fruitful exchanges where a part of this work has been presented.

\section{APPENDIX A: REVIEW OF GEODESIC EQUATION AND SPIN EVOLUTION OF SPINNING OBJECT}

In this Appendix we review the geodesic equation for the spinning object as well as the time evolution of the spin. These equations are known as Mathisson-Papapetrou equations $[33,34]$ in general relativity and the covariant form of those are discovered by Tulczyjew and Dixon [26,27]. The Mathisson-Papapetrou-Dixon equations take the following form:

$$
\begin{aligned}
& \frac{D P^{\mu}}{D \sigma}=-\frac{1}{2} R^{\mu}{ }_{\nu \rho \sigma} u^{\nu} \Sigma^{\rho \sigma}, \\
& \frac{D \Sigma^{\mu \nu}}{D \sigma}=P^{\mu} u^{\nu}-P^{\nu} u^{\mu},
\end{aligned}
$$

where $P^{\mu}$ is the kinematical momentum and $\Sigma^{\mu \nu}$ is the spin angular momentum of the object measured along the world line as a function of affine parameter $\sigma$. The four velocity is denoted by $u^{\mu}$ and defined as $u^{\mu}=\frac{d x^{\mu}}{d \sigma}$, for $x^{\mu}(\sigma)$ representing the world line and $u^{\mu} u_{\mu}=-1$ if $\sigma$ is the proper time. $\frac{D}{D \sigma}$ represents the covariant derivative along the world line. Let us define the kinematic mass $m \equiv-P \cdot u$, which is in general not a constant of motion [45]. Now contracting with $u_{\nu}$ from Eq. (A2) we get

$$
P^{\mu}=m u^{\mu}-\frac{D \Sigma^{\mu \nu}}{D \sigma} u_{\nu}
$$

To understand how the kinematical mass evolves along the trajectory, let us take the derivative over $m$ with respect to $\sigma$,

$\frac{d m}{d \sigma}=\frac{D m}{D \sigma}=-\frac{D P^{\mu}}{D \sigma} u_{\mu}-P^{\mu} \frac{D u_{\mu}}{D \sigma}=\frac{D u_{\mu}}{D \sigma} \frac{D \Sigma^{\mu \nu}}{D \sigma} u_{\nu}$,

where to get the last expression after equality, we substituted the results of Eqs. (A1) and (A2). Now we substitute the expression of $P^{\mu}$ in Eq. (A1) and use the expressions in Eqs. (A4) and (A2). After this substitution we simplify Eq. (A1) using $u_{\alpha} \frac{D u^{\alpha}}{D \sigma}=0$ and get the following simplified trajectory equation:

$$
m \frac{D u^{\mu}}{D \sigma}-\frac{D^{2} \Sigma^{\mu \nu}}{D \sigma^{2}} u_{\nu}=-\frac{1}{2} R_{\nu \rho \sigma}^{\mu} u^{\nu} \Sigma^{\rho \sigma}
$$

Note that to derive the above trajectory equation we do not have to use any spin supplementarity condition (SSC).

Now let us focus on the simplification of the spin evolution equation given in Eq. (A2), using the trajectory equation (A5) and spin supplementarity condition $u_{\mu} \Sigma^{\mu \nu}=0$. We start by substituting the expression of $P^{\mu}$ in Eq. (A2) and get

$$
\frac{D \Sigma^{\mu \nu}}{D \sigma}=-\frac{D \Sigma^{\mu \alpha}}{D \sigma} u_{\alpha} u^{\nu}+\frac{D \Sigma^{\nu \alpha}}{D \sigma} u_{\alpha} u^{\mu} .
$$

Now using the definition of the covariant derivative on the spin tensor, moving the $\sigma$ derivatives from $\Sigma$ to $u$, and simplifying using spin supplementary condition $u_{\mu} \Sigma^{\mu \nu}=0,{ }^{9}$ the above equation takes the following form:

$$
\begin{aligned}
& \frac{d \Sigma^{\mu \nu}}{d \sigma}+\Gamma_{\alpha \beta}^{\mu} \Sigma^{\alpha \nu} u^{\beta}+\Gamma_{\alpha \beta}^{\nu} \Sigma^{\mu \alpha} u^{\beta} \\
& =\Sigma^{\mu \alpha} \frac{d u_{\alpha}}{d \sigma} u^{\nu}-\Sigma^{\nu \alpha} \frac{d u_{\alpha}}{d \sigma} u^{\mu}-\Gamma_{\rho \sigma}^{\alpha} \Sigma^{\mu \rho} u^{\sigma} u_{\alpha} u^{\nu} \\
& \quad+\Gamma_{\rho \sigma}^{\alpha} \Sigma^{\nu \rho} u^{\sigma} u_{\alpha} u^{\mu} .
\end{aligned}
$$

Substituting the trajectory equation (A5) in the RHS of the above equation, we get

$$
\begin{aligned}
& \frac{d \Sigma^{\mu \nu}}{d \sigma}+\Gamma_{\alpha \beta}^{\mu} \Sigma^{\alpha \nu} u^{\beta}+\Gamma_{\alpha \beta}^{\nu} \Sigma^{\mu \alpha} u^{\beta} \\
& =\frac{1}{m}\left(\Sigma^{\mu \alpha} u^{\nu}-\Sigma^{\nu \alpha} u^{\mu}\right)\left(\frac{D^{2} \Sigma_{\alpha \beta}}{D \sigma^{2}} u^{\beta}-\frac{1}{2} R_{\alpha \beta \rho \sigma} u^{\beta} \Sigma^{\rho \sigma}\right) .
\end{aligned}
$$

In the above equation the left-hand side (LHS) is basically $\frac{D \Sigma^{\mu \nu}}{D \sigma}$, which is equal to terms quadratic in spin as written in the RHS. This above result also implies that $\frac{D^{2} \Sigma^{\mu \nu}}{D \sigma^{2}}$ in Eq. (A5) is also quadratic in spin. Hence if we are interested in the trajectory and spin evolution equations which are linear in spin, ${ }^{10}$ Eqs. (A5) and (A8) simplify to

\footnotetext{
${ }^{9}$ In the literature, we find two kinds of SSC, which are $u_{\mu} \Sigma^{\mu \nu}=0$ and $P_{\mu} \Sigma^{\mu \nu}=0$. Different choices of SSC, will modify the $\mathcal{O}\left(\Sigma^{2}\right)$ terms in the RHS of Eqs. (A9) and (A10), which are irrelevant for our analysis.

${ }^{10}$ Here we want to emphasize that we are not making any small spin approximation while ignoring terms quadratic in spin. We are ignoring those terms as those contribute at order $\mathcal{O}\left(G^{2}\right)$ to the correction of the trajectory and spin in our analysis of Sec. III C and would not affect our $\mathcal{O}\left(G^{2}\right)$ gravitational waveform.
} 
$\frac{d^{2} x^{\mu}}{d \sigma^{2}}+\Gamma_{\rho \sigma}^{\mu} \frac{d x^{\rho}}{d \sigma} \frac{d x^{\sigma}}{d \sigma}=-\frac{1}{2 m} R_{\nu \rho \sigma}^{\mu} \frac{d x^{\nu}}{d \sigma} \Sigma^{\rho \sigma}+\mathcal{O}\left(\Sigma^{2}\right)$

and

$\frac{d \Sigma^{\mu \nu}}{d \sigma}+\Gamma_{\alpha \beta}^{\mu} \Sigma^{\alpha \nu} \frac{d x^{\beta}}{d \sigma}+\Gamma_{\alpha \beta}^{\nu} \Sigma^{\mu \alpha} \frac{d x^{\beta}}{d \sigma}=0+\mathcal{O}\left(\Sigma^{2}\right)$.

Now we show that the above equations also follow from the covariant conservation of the canonical version of the matter energy-momentum tensor in Eq. (3.10). For a given world-line action $S_{X}$ of a moving object in the gravitational background with metric $g_{\mu \nu}(x)$, the canonical matter energy-momentum tensor is defined by

$$
T_{c}^{X \alpha \beta}(x) \equiv \frac{2}{\sqrt{-g}} \frac{\delta S_{X}}{\delta g_{\alpha \beta}(x)},
$$

which satisfies the following conservation equation:

$$
\nabla_{\alpha} T_{c}^{X \alpha \beta}(x)=0 .
$$

In the above expressions, $g \equiv \operatorname{det}\left(g_{\mu \nu}\right)$ and $\nabla_{\alpha}$ represents the covariant derivative with background metric $g_{\mu \nu}(x)$. Let $\phi_{\beta}(x)$ be an arbitrary rank-1 tensor with a sufficient falloff at the boundary of spacetime, such that $\left[\sqrt{-g} T_{c}^{X \alpha \beta}(x) \phi_{\beta}(x)\right]_{|x| \rightarrow \infty}=0$ will be satisfied. Now start with the following identity:

$$
\begin{aligned}
\int d^{4} x \sqrt{-g} \nabla_{\alpha}\left(T_{c}^{X \alpha \beta}(x) \phi_{\beta}(x)\right) & \left.=\int d^{4} x \sqrt{-g}\left[\left(\nabla_{\alpha} T_{c}^{X \alpha \beta}(x)\right) \phi_{\beta}(x)\right)+T_{c}^{X \alpha \beta}(x) \nabla_{(\alpha} \phi_{\beta)}(x)\right] \\
& \Rightarrow \int d^{4} x \sqrt{-g} T_{c}^{X \alpha \beta}(x) \nabla_{(\alpha} \phi_{\beta)}(x)=0 .
\end{aligned}
$$

Above to get the last line we used the fact that the LHS of the first line is a boundary term that vanishes and the first term in the RHS of the first line vanishes due to the conservation of the matter energy-momentum tensor. In our convention, the relation between the matter energy-momentum tensor given in Eq. (3.10) and the canonical energy-momentum tensor is $T^{X \alpha \beta}(x) \equiv \sqrt{-g} T_{c}^{X \alpha \beta}(x)$. Now after substituting the matter energy-momentum tensor of Eq. (3.10) for a single particle in the above relation, we get

$$
\begin{aligned}
& \int d \sigma \int d^{4} x\left[m \frac{d X^{\alpha}(\sigma)}{d \sigma} \frac{d X^{\beta}(\sigma)}{d \sigma} \delta^{(4)}(x-X(\sigma))+\frac{d X^{(\alpha}(\sigma)}{d \sigma} \Sigma^{\beta) \gamma}(\sigma)\right. \\
& \left.\times \partial_{\gamma} \delta^{(4)}(x-X(\sigma))+\Gamma_{\gamma \delta}^{(\alpha}(X) \Sigma^{\beta) \gamma}(\sigma) \frac{d X^{\delta}(\sigma)}{d \sigma} \delta^{(4)}(x-X(\sigma))\right] \\
& \quad \times\left\{\partial_{(\alpha} \phi_{\beta)}(x)-\Gamma_{\alpha \beta}^{\rho}(x) \phi_{\rho}(x)\right\}=0 .
\end{aligned}
$$

In the above expression, we first perform integration by parts to remove the derivative over the delta function and then do the spacetime integration using the delta function. Next, after using the identity $\frac{d X^{\alpha}}{d \sigma} \frac{\partial}{\partial X^{\alpha}}=\frac{d}{d \sigma}$ and integration by parts for the derivatives with respect to $\sigma$, we get

$$
\begin{aligned}
& \int d \sigma \phi_{\rho}(X)\left[-m \frac{d^{2} X^{\rho}(\sigma)}{d \sigma^{2}}-m \Gamma_{\alpha \beta}^{\rho}(X) \frac{d X^{\alpha}(\sigma)}{d \sigma} \frac{d X^{\beta}(\sigma)}{d \sigma}\right. \\
& \left.+\partial_{\gamma} \Gamma_{\alpha \beta}^{\rho}(X) \frac{d X^{\alpha}(\sigma)}{d \sigma} \Sigma^{\beta \gamma}(\sigma)-\Gamma_{\alpha \beta}^{\rho}(X) \Gamma_{\gamma \delta}^{\alpha}(X) \frac{d X^{\delta}(\sigma)}{d \sigma} \Sigma^{\beta \gamma}(\sigma)\right] \\
& +\frac{1}{2} \int d \sigma \partial_{\gamma} \phi_{\rho}(X)\left[\frac{d \Sigma^{\gamma \gamma}(\sigma)}{d \sigma}+\Gamma_{\alpha \beta}^{\rho}(X) \frac{d X^{\beta}(\sigma)}{d \sigma} \Sigma^{\alpha \gamma}(\sigma)-\Gamma_{\alpha \beta}^{\gamma}(X) \frac{d X^{\beta}(\sigma)}{d \sigma} \Sigma^{\alpha \rho}(\sigma)\right]=0 .
\end{aligned}
$$

Since $\phi_{\rho}(x)$ is an arbitrary rank-1 tensor, in the above expression the coefficients of $\phi_{\rho}(X)$ and $\partial_{\gamma} \phi_{\rho}(X)$ will vanish individually. Hence replacing $\rho \rightarrow \mu$ and $\gamma \rightarrow \nu$ and using the antisymmetry property of $\Sigma(\sigma)$ we get the following two equations from the above expression:

$$
\begin{aligned}
& \frac{d^{2} X^{\mu}(\sigma)}{d \sigma^{2}}+\Gamma_{\alpha \beta}^{\mu}(X) \frac{d X^{\alpha}(\sigma)}{d \sigma} \frac{d X^{\beta}(\sigma)}{d \sigma}=-\frac{1}{2 m} R_{\alpha \beta \gamma}^{\mu}(X) \frac{d X^{\alpha}(\sigma)}{d \sigma} \Sigma^{\beta \gamma}(\sigma) \\
& \frac{d \Sigma^{\mu \nu}(\sigma)}{d \sigma}+\Gamma_{\alpha \beta}^{\mu}(X) \frac{d X^{\alpha}(\sigma)}{d \sigma} \Sigma^{\beta \nu}(\sigma)+\Gamma_{\alpha \beta}^{\nu}(X) \frac{d X^{\alpha}(\sigma)}{d \sigma} \Sigma^{\mu \beta}(\sigma)=0
\end{aligned}
$$


where

$$
R_{\alpha \beta \gamma}^{\mu}(X)=\partial_{\beta} \Gamma_{\alpha \gamma}^{\mu}(X)-\partial_{\gamma} \Gamma_{\alpha \beta}^{\mu}(X)+\Gamma_{\alpha \gamma}^{\delta}(X) \Gamma_{\delta \beta}^{\mu}(X)-\Gamma_{\alpha \beta}^{\delta}(X) \Gamma_{\delta \gamma}^{\mu}(X) .
$$

Now if we compare the above equations with Eqs. (A9) and (A10), we observe that they are identical. This also proves that the geodesic equation and spin evolution equation are consistent with our matter energy-momentum tensor.

\section{APPENDIX B: DETAIL ANALYSIS OF GRAVITATIONAL ENERGY-MOMENTUM TENSOR GIVEN IN EQ. (3.53)}

To evaluate the expression in Eq. (3.53) we need to use the results of the following three integrals:

$$
\begin{aligned}
J_{1}^{\alpha \beta}= & \int_{\omega}^{L^{-1}} \frac{d^{4} \ell}{(2 \pi)^{4}}\left[G_{r}(-\ell)\right]^{2} \frac{1}{p_{a} \cdot \ell+i \epsilon} \frac{1}{p_{b} \cdot \ell-i \epsilon} \ell^{\alpha} \ell^{\beta} \\
= & -\frac{1}{2}\left[p_{a}^{\alpha} \frac{\partial}{\partial p_{a \beta}}+p_{b}^{\alpha} \frac{\partial}{\partial p_{b \beta}}+\eta^{\alpha \beta}\right] \int_{\omega}^{L^{-1}} \frac{d^{4} \ell}{(2 \pi)^{4}} G_{r}(-\ell) \frac{1}{p_{a} \cdot \ell+i \epsilon} \frac{1}{p_{b} \cdot \ell-i \epsilon} \\
= & \frac{1}{8 \pi} \delta_{\eta_{a} \eta_{b}, 1} \ln \left\{L\left(\omega+i \epsilon \eta_{a}\right)\right\} \frac{1}{\left[\left(p_{a} \cdot p_{b}\right)^{2}-p_{a}^{2} p_{b}^{2}\right]^{3 / 2}}\left[p_{a} \cdot p_{b}\left\{p_{a}^{\alpha} p_{b}^{\beta}+p_{b}^{\alpha} p_{a}^{\beta}\right\}-p_{b}^{2} p_{a}^{\alpha} p_{a}^{\beta}\right. \\
& \left.-p_{a}^{2} p_{b}^{\alpha} p_{b}^{\beta}-\eta^{\alpha \beta}\left\{\left(p_{a} \cdot p_{b}\right)^{2}-p_{a}^{2} p_{b}^{2}\right\}\right], \\
J_{2}^{\alpha \beta \gamma}= & \int_{\omega}^{L^{-1}} \frac{d^{4} \ell}{(2 \pi)^{4}}\left[G_{r}(-\ell)\right]^{2} \frac{1}{p_{b} \cdot \ell-i \epsilon} \frac{1}{\left(p_{a} \cdot \ell+i \epsilon\right)^{2}} \ell^{\alpha} \ell^{\beta} \ell^{\gamma} \\
= & \frac{1}{2}\left[p_{b}^{\gamma} \frac{\partial}{\partial p_{b \alpha}} \frac{\partial}{\partial p_{a \beta}}+p_{a}^{\gamma} \frac{\partial}{\partial p_{a \alpha}} \frac{\partial}{\partial p_{a \beta}}+\left(\eta^{\alpha \gamma} \frac{\partial}{\partial p_{a \beta}}+\eta^{\beta \gamma} \frac{\partial}{\partial p_{a \alpha}}\right)\right] \\
& \times \int_{\omega}^{L^{-1}} \frac{d^{4} \ell}{(2 \pi)^{4}} G_{r}(-\ell) \frac{1}{p_{a} \cdot \ell+i \epsilon} \frac{1}{p_{b} \cdot \ell-i \epsilon} \\
= & \frac{1}{8 \pi} \delta_{\eta_{a} \eta_{b}, 1} \ln \left\{L\left(\omega+i \epsilon \eta_{a}\right)\right\} \frac{1}{\left[\left(p_{a} \cdot p_{b}\right)^{2}-p_{a}^{2} p_{b}^{2}\right]^{5 / 2}}\left[3\left(p_{a} \cdot p_{b}\right)^{2} p_{a}^{\alpha} p_{b}^{\beta} p_{b}^{\gamma}-3 p_{a}^{2} p_{a} \cdot p_{b} p_{b}^{\alpha} p_{b}^{\beta} p_{b}^{\gamma}\right. \\
& -3 p_{b}^{2} p_{a} \cdot p_{b} p_{a}^{\alpha} p_{a}^{\beta} p_{b}^{\gamma}+3 p_{a}^{2} p_{b}^{2} p_{b}^{\alpha} p_{a}^{\beta} p_{b}^{\gamma}+3\left(p_{a} \cdot p_{b}\right)^{2} p_{b}^{\alpha} p_{b}^{\beta} p_{a}^{\gamma}-3 p_{b}^{2} p_{a} \cdot p_{b} p_{a}^{\alpha} p_{b}^{\beta} p_{a}^{\gamma} \\
- & \left.3 p_{b}^{2} p_{a} \cdot p_{b} p_{b}^{\alpha} p_{a}^{\beta} p_{a}^{\gamma}+3\left(p_{b}^{2}\right)^{2} p_{a}^{\alpha} p_{a}^{\beta} p_{a}^{\gamma}\right] \\
- & \frac{1}{8 \pi} \delta_{\eta_{a} \eta_{b}, 1} \ln \left\{L\left(\omega+i \epsilon \eta_{a}\right)\right\} \frac{1}{\left[\left(p_{a} \cdot p_{b}\right)^{2}-p_{a}^{2} p_{b}^{2}\right]^{3 / 2}}\left[p_{a}^{\alpha} p_{b}^{\beta} p_{b}^{\gamma}-2 p_{b}^{\alpha} p_{a}^{\beta} p_{b}^{\gamma}+p_{a} \cdot p_{b} \eta^{\alpha \beta} p_{b}^{\gamma}\right. \\
+ & \left.p_{b}^{\alpha} p_{b}^{\beta} p_{a}^{\gamma}-p_{b}^{2} \eta^{\alpha \beta} p_{a}^{\gamma}+p_{a} \cdot p_{b} p_{b}^{\beta} \eta^{\alpha \gamma}-p_{b}^{2} p_{a}^{\beta} \eta^{\alpha \gamma}+p_{a} \cdot p_{b} p_{b}^{\alpha} \eta^{\beta \gamma}-p_{b}^{2} p_{a}^{\alpha} \eta^{\beta \gamma}\right], \\
& J_{3}^{\alpha}=\int_{\omega}^{L^{-1}} \frac{d^{4} \ell}{(2 \pi)^{4}} G_{r}(-\ell) \frac{1}{p_{b} \cdot \ell-i \epsilon} \frac{1}{\left(p_{a} \cdot \ell+i \epsilon\right)^{2}} \ell^{\alpha} \\
\eta_{a} \eta_{b}, 1 & \ln \left\{L\left(\omega+i \epsilon \eta_{a}\right)\right\} \frac{1}{\left[\left(p_{a} \cdot p_{b}\right)^{2}-p_{a}^{2} p_{b}^{2}\right]^{3 / 2}}\left[p_{a} \cdot p_{b} p_{b}^{\alpha}-p_{b}^{2} p_{a}^{\alpha}\right] . \\
& \\
&
\end{aligned}
$$

To evaluate the expression in Eq. (3.53), we divide it into a sum over four integrals:

$$
\Delta_{(1)}^{(\omega \ln \omega)} \hat{T}_{2}^{h \mu \nu}(k)=\mathcal{L}_{1}^{\mu \nu}+\mathcal{L}_{2}^{\mu \nu}+\mathcal{L}_{3}^{\mu \nu}+\mathcal{L}_{4}^{\mu \nu}
$$

The first three lines after the equality in Eq. (3.53) take the following form after removing the terms containing $p_{a} . \ell$ and $p_{b} . \ell$ in the numerator ${ }^{11}$ :

\footnotetext{
${ }^{11}$ We do not keep the terms containing $p_{a} . \ell$ or $p_{b} . \ell$ in the numerator as they cancel with the denominator of the integrand, and then it can be shown that the integration result for those terms vanishes after the $\ell^{0}$ contour integration.
} 


$$
\begin{aligned}
\mathcal{L}_{1}^{\mu \nu}= & \frac{i}{2}(8 \pi G) \sum_{a, b=1}^{M+N} \int_{\omega}^{L^{-1}} \frac{d^{4} \ell}{(2 \pi)^{4}}\left\{G_{r}(-\ell)\right\}^{3}(-2 k \cdot \ell) \frac{1}{p_{b} \cdot \ell-i \epsilon} \frac{1}{p_{a} \cdot \ell+i \epsilon} \\
& \times\left[2 \ell^{\mu} \ell^{\nu} p_{a} \cdot p_{b} p_{a \alpha} J_{b}^{\alpha \beta} \ell_{\beta}-p_{a}^{2} \ell^{\mu} \ell^{\nu} p_{b \alpha} J_{b}^{\alpha \beta} \ell_{\beta}+2 \ell^{2} p_{b}^{\mu} p_{a}^{\nu} p_{a \alpha} J_{b}^{\alpha \beta} \ell_{\beta}+2 \ell^{2} p_{a} \cdot p_{b} p_{a}^{\nu} J_{b}^{\mu \alpha} \ell_{\alpha}\right. \\
& \left.-\eta^{\mu \nu} \ell^{2} p_{a} \cdot p_{b} p_{a \alpha} J_{b}^{\alpha \beta} \ell_{\beta}+\frac{1}{2} p_{a}^{2} \eta^{\mu \nu} \ell^{2} p_{b \alpha} J_{b}^{\alpha \beta} \ell_{\beta}-2 \ell^{2} p_{a}^{\mu} p_{a}^{\nu} p_{b \alpha} J_{b}^{\alpha \beta} \ell_{\beta}\right] \\
& -\frac{i}{2}(8 \pi G) \sum_{a, b=1}^{M+N} \int_{\omega}^{L^{-1}} \frac{d^{4} \ell}{(2 \pi)^{4}}\left\{G_{r}(-\ell)\right\}^{3}(-2 k \cdot \ell) \frac{1}{p_{b} \cdot \ell-i \epsilon} \frac{1}{p_{a} \cdot \ell+i \epsilon} \\
& \times\left[2 \ell^{\mu} \ell^{\nu} p_{a} \cdot p_{b} p_{b \rho} J_{a}^{\rho \sigma} \ell_{\sigma}-p_{b}^{2} \ell^{\mu} \ell^{\nu} p_{a \rho} J_{a}^{\rho \sigma} \ell_{\sigma}+\frac{1}{2} p_{b}^{2} \ell^{2} \eta^{\mu \nu} p_{a \rho} J_{a}^{\rho \sigma} \ell_{\sigma}-\ell^{2} p_{b}^{2} p_{a}^{\mu} J_{a}^{\nu \sigma} \ell_{\sigma}\right. \\
& \left.-\ell^{2} p_{b}^{2} p_{a}^{\nu} J_{a}^{\mu \sigma} \ell_{\sigma}+2 \ell^{2} p_{a} \cdot p_{b} p_{b}^{\mu} J_{a}^{\nu \sigma} \ell_{\sigma}+2 \ell^{2} p_{a}^{\nu} p_{b}^{\mu} p_{b \rho} J_{a}^{\rho \sigma} \ell_{\sigma}-\ell^{2} p_{a} \cdot p_{b} \eta^{\mu \nu} p_{b \rho} J_{a}^{\rho \sigma} \ell_{\sigma}\right] .
\end{aligned}
$$

In the above expression the particle indices $a$ and $b$ are dummy, so we can exchange $a \leftrightarrow b$ in the second integral above. In doing so we observe that many terms are the same within the square bracket of the first and second integrands, but there is a relative sign between the two integrals; hence those terms will cancel each other. ${ }^{12}$ After canceling those terms we combine the two integrals into one and get the following expression:

$$
\begin{aligned}
\mathcal{L}_{1}^{\mu \nu}= & -\frac{i}{2}(8 \pi G) \sum_{a, b=1}^{M+N} \int_{\omega}^{L^{-1}} \frac{d^{4} \ell}{(2 \pi)^{4}}\left\{G_{r}(-\ell)\right\}^{2}(-2 k \cdot \ell) \frac{1}{p_{b} \cdot \ell-i \epsilon} \frac{1}{p_{a} \cdot \ell+i \epsilon} \\
& \times\left[2 p_{b}^{\mu} p_{a}^{\nu} p_{a \alpha} J_{b}^{\alpha \beta} \ell_{\beta}-2 p_{a}^{\mu} p_{a}^{\nu} p_{b \alpha} J_{b}^{\alpha \beta} \ell_{\beta}+p_{a}^{2} p_{b}^{\mu} J_{b}^{\nu \sigma} \ell_{\sigma}+p_{a}^{2} p_{b}^{\nu} J_{b}^{\mu \sigma} \ell_{\sigma}-2 p_{b}^{\nu} p_{a}^{\mu} p_{a \rho} J_{b}^{\rho \sigma} \ell_{\sigma}\right. \\
& \left.+2 p_{a} \cdot p_{b} p_{a}^{\nu} J_{b}^{\mu \alpha} \ell_{\alpha}-2 p_{a} \cdot p_{b} p_{a}^{\mu} J_{b}^{\nu \alpha} \ell_{\alpha}\right] .
\end{aligned}
$$

The fourth to sixth lines after the equality in Eq. (3.53) take the following form after removing the terms containing $\left(p_{a} \cdot \ell\right)^{2}$ and $p_{b} \cdot l$ in the numerator:

$$
\begin{aligned}
\mathcal{L}_{2}^{\mu \nu}= & \frac{i}{2}(8 \pi G) \sum_{a, b=1}^{M+N} \int_{\omega}^{L^{-1}} \frac{d^{4} \ell}{(2 \pi)^{4}}\left\{G_{r}(-\ell)\right\}^{2}\left(p_{a} \cdot k\right) \frac{1}{p_{b} \cdot \ell-i \epsilon} \frac{1}{\left(p_{a} \cdot \ell+i \epsilon\right)^{2}} \\
& \times\left[2 \ell^{\mu} \ell^{\nu} p_{a} \cdot p_{b} p_{a \alpha} J_{b}^{\alpha \beta} \ell_{\beta}-p_{a}^{2} \ell^{\mu} \ell^{\nu} p_{b \alpha} J_{b}^{\alpha \beta} \ell_{\beta}+2 \ell^{2} p_{b}^{\mu} p_{a}^{\nu} p_{a \alpha} J_{b}^{\alpha \beta} \ell_{\beta}+2 \ell^{2} p_{a} \cdot p_{b} p_{a}^{\nu} J_{b}^{\mu \alpha} \ell_{\alpha}\right. \\
& \left.-\eta^{\mu \nu} \ell^{2} p_{a} \cdot p_{b} p_{a \alpha} J_{b}^{\alpha \beta} \ell_{\beta}+\frac{1}{2} p_{a}^{2} \eta^{\mu \nu} \ell^{2} p_{b \alpha} J_{b}^{\alpha \beta} \ell_{\beta}-2 \ell^{2} p_{a}^{\mu} p_{a}^{\nu} p_{b \alpha} J_{b}^{\alpha \beta} \ell_{\beta}+2 \ell^{\mu} p_{a}^{\nu} p_{a} \cdot \ell p_{b \alpha} J_{b}^{\alpha \beta} \ell_{\beta}\right] \\
& -\frac{i}{2}(8 \pi G) \sum_{a, b=1}^{M+N} \int_{\omega}^{L^{-1}} \frac{d^{4} \ell}{(2 \pi)^{4}}\left\{G_{r}(-\ell)\right\}^{2}\left(p_{a} \cdot k\right) \frac{1}{p_{b} \cdot \ell-i \epsilon} \frac{1}{\left(p_{a} \cdot \ell+i \epsilon\right)^{2}} \\
& \times\left[2 \ell^{\mu} \ell^{\nu} p_{a} \cdot p_{b} p_{b \rho} J_{a}^{\rho \sigma} \ell_{\sigma}-p_{b}^{2} \ell^{\mu} \ell^{\nu} p_{a \rho} J_{a}^{\rho \sigma} \ell_{\sigma}+\frac{1}{2} p_{b}^{2} \ell^{2} \eta^{\mu \nu} p_{a \rho} J_{a}^{\rho \sigma} \ell_{\sigma}-\ell^{2} p_{b}^{2} p_{a}^{\mu} J_{a}^{\nu \sigma} \ell_{\sigma}\right. \\
& -\ell^{2} p_{b}^{2} p_{a}^{\nu} J_{a}^{\mu \sigma} \ell_{\sigma}+2 \ell^{2} p_{a} \cdot p_{b} p_{b}^{\mu} J_{a}^{\nu \sigma} \ell_{\sigma}+2 \ell^{2} p_{a}^{\nu} p_{b}^{\mu} p_{b \rho} J_{a}^{\rho \sigma} \ell_{\sigma}-\ell^{2} p_{a} \cdot p_{b} \eta^{\mu \nu} p_{b \rho} J_{a}^{\rho \sigma} \ell_{\sigma} \\
& \left.+p_{b}^{2} \ell^{\mu} p_{a} \cdot \ell J_{a}^{\nu \alpha} \ell_{\alpha}\right] .
\end{aligned}
$$

The seventh to ninth lines after the equality in Eq. (3.53) take the following form after removing the terms containing $p_{a} . \ell$ and $p_{b} . \ell$ in the numerator,

\footnotetext{
${ }^{12}$ Obviously for the canceled terms between the two integrals there will be a sign difference in front of $i \epsilon$ in $\left\{p_{a} \cdot \ell \pm i \epsilon\right\}^{-1}$ and $\left\{p_{b} . \ell \pm i \epsilon\right\}^{-1}$ but the contribution turns out to be the same as only the relative signs in front of $i \epsilon$ between the two denominators matter.
} 


$$
\begin{aligned}
& \mathcal{L}_{3}^{\mu \nu}=\frac{i}{2}(8 \pi G) \sum_{a, b=1}^{M+N} \int_{\omega}^{L^{-1}} \frac{d^{4} \ell}{(2 \pi)^{4}}\left\{G_{r}(-\ell)\right\}^{2} \frac{1}{p_{b} \cdot \ell-i \epsilon} \frac{1}{p_{a} \cdot \ell+i \epsilon} \\
& \times\left[-2 \ell^{\mu} k^{\nu} p_{a} \cdot p_{b} p_{a \alpha} J_{b}^{\alpha \beta} \ell_{\beta}+p_{a}^{2} k^{\nu} \ell^{\mu} p_{b \alpha} J_{b}^{\alpha \beta} \ell_{\beta}-4 k^{\mu} \ell^{\nu} p_{a} \cdot p_{b} p_{a \alpha} J_{b}^{\alpha \beta} \ell_{\beta}\right. \\
& +2 p_{a}^{2} k^{\mu} \ell^{\nu} p_{b \alpha} J_{b}^{\alpha \beta} \ell_{\beta}+2 p_{a} \cdot p_{b} p_{a}^{\mu} \ell^{\nu} k_{\alpha} J_{b}^{\alpha \beta} \ell_{\beta}+2 p_{b} \cdot k p_{a}^{\mu} \ell^{\nu} p_{a \alpha} J_{b}^{\alpha \beta} \ell_{\beta} \\
& -2 p_{a} \cdot k p_{a}^{\mu} \ell^{\nu} p_{b \alpha} J_{b}^{\alpha \beta} \ell_{\beta}-p_{a}^{2} p_{b}^{\mu} \ell^{\nu} k_{\alpha} J_{b}^{\alpha \beta} \ell_{\beta}-p_{a}^{2} p_{b} \cdot k \ell^{\nu} J_{b}^{\mu \alpha} \ell_{\alpha} \\
& +2 p_{a} \cdot p_{b} p_{a}^{\nu} \ell^{\mu} k_{\alpha} J_{b}^{\alpha \beta} \ell_{\beta}+2 p_{b} \cdot k p_{a}^{\nu} \ell^{\mu} p_{a \alpha} J_{b}^{\alpha \beta} \ell_{\beta}-2 p_{a} \cdot k p_{a}^{\nu} \ell^{\mu} p_{b \alpha} J_{b}^{\alpha \beta} \ell_{\beta} \\
& +6 k . \ell p_{a}^{\mu} p_{a}^{\nu} p_{b \alpha} J_{b}^{\alpha \beta} \ell_{\beta}-\frac{5}{2} k . \ell p_{a}^{2} \eta^{\mu \nu} p_{b \alpha} J_{b}^{\alpha \beta} \ell_{\beta}-2 k . \ell p_{a} \cdot p_{b} p_{a}^{\mu} J_{b}^{\nu \alpha} \ell_{\alpha} \\
& -2 k . \ell p_{b}^{\nu} p_{a}^{\mu} p_{a \alpha} J_{b}^{\alpha \beta} \ell_{\beta}+k . \ell p_{a}^{2} p_{b}^{\mu} J_{b}^{\nu \alpha} \ell_{\alpha}+k . \ell p_{a}^{2} p_{b}^{\nu} J_{b}^{\mu \alpha} \ell_{\alpha} \\
& \left.-4 k . \ell p_{b}^{\mu} p_{a}^{\nu} p_{a \alpha} J_{b}^{\alpha \beta} \ell_{\beta}-4 k . \ell p_{a} \cdot p_{b} p_{a}^{\nu} J_{b}^{\mu \alpha} \ell_{\alpha}+5 k \cdot \ell \eta^{\mu \nu} p_{a} \cdot p_{b} p_{a \alpha} J_{b}^{\alpha \beta} \ell_{\beta}\right] \\
& -\frac{i}{2}(8 \pi G) \sum_{a, b=1}^{M+N} \int_{\omega}^{L^{-1}} \frac{d^{4} \ell}{(2 \pi)^{4}}\left\{G_{r}(-\ell)\right\}^{2} \frac{1}{p_{b} \cdot \ell-i \epsilon} \frac{1}{p_{a} \cdot \ell+i \epsilon} \\
& \times\left[-2 p_{a} \cdot p_{b} \ell^{\mu} k^{\nu} p_{b \alpha} J_{a}^{\alpha \beta} \ell_{\beta}+p_{b}^{2} \ell^{\mu} k^{\nu} p_{a \alpha} J_{a}^{\alpha \beta} \ell_{\beta}-4 k^{\mu} \ell^{\nu} p_{a} \cdot p_{b} p_{b \alpha} J_{a}^{\alpha \beta} \ell_{\beta}\right. \\
& +2 p_{b}^{2} k^{\mu} \ell^{\nu} p_{a \alpha} J_{a}^{\alpha \beta} \ell_{\beta}+2 p_{b} \cdot k p_{a} \cdot p_{b} \ell^{\nu} J_{a}^{\mu \alpha} \ell_{\alpha}+2 p_{b} \cdot k p_{a}^{\mu} \ell^{\nu} p_{b \alpha} J_{a}^{\alpha \beta} \ell_{\beta} \\
& -2 p_{b} \cdot k p_{b}^{\mu} \ell^{\nu} p_{a \alpha} J_{a}^{\alpha \beta} \ell_{\beta}-p_{b}^{2} p_{a} \cdot k \ell^{\nu} J_{a}^{\mu \alpha} \ell_{\alpha}-p_{b}^{2} \ell^{\nu} p_{a}^{\mu} k_{\alpha} J_{a}^{\alpha \beta} \ell_{\beta} \\
& +2 p_{a} \cdot p_{b} p_{b} \cdot k \ell^{\mu} J_{a}^{\nu \alpha} \ell_{\alpha}+2 p_{a}^{\nu} \ell^{\mu} p_{b} \cdot k p_{b \alpha} J_{a}^{\alpha \beta} \ell_{\beta}-p_{b}^{2} p_{a} \cdot k \ell^{\mu} J_{a}^{\nu \alpha} \ell_{\alpha} \\
& -p_{b}^{2} \ell^{\mu} p_{a}^{\nu} k_{\alpha} J_{a}^{\alpha \beta} \ell_{\beta}+2 p_{b}^{\mu} p_{b}^{\nu} k \cdot \ell p_{a \alpha} J_{a}^{\alpha \beta} \ell_{\beta}-\frac{5}{2} p_{b}^{2} k \cdot \ell \eta^{\mu \nu} p_{a \alpha} J_{a}^{\alpha \beta} \ell_{\beta} \\
& -4 k . \ell p_{a} \cdot p_{b} p_{b}^{\mu} J_{a}^{\nu \alpha} \ell_{\alpha}-4 k . \ell p_{b}^{\mu} p_{a}^{\nu} p_{b \alpha} J_{a}^{\alpha \beta} \ell_{\beta}-2 k \cdot \ell p_{b}^{\nu} p_{a} \cdot p_{b} J_{a}^{\mu \alpha} \ell_{\alpha} \\
& -2 k . \ell p_{b}^{\nu} p_{a}^{\mu} p_{b \alpha} J_{a}^{\alpha \beta} \ell_{\beta}+3 k \cdot \ell p_{b}^{2} p_{a}^{\nu} J_{a}^{\mu \alpha} \ell_{\alpha}+3 k . \ell p_{b}^{2} p_{a}^{\mu} J_{a}^{\nu \alpha} \ell_{\alpha} \\
& \left.+5 \eta^{\mu \nu} p_{a} \cdot p_{b} k \cdot \ell p_{b \alpha} J_{a}^{\alpha \beta} \ell_{\beta}\right] \text {. }
\end{aligned}
$$

In the above expression the particle indices $a$ and $b$ are dummy, so we can exchange $a \leftrightarrow b$ in the second integral above. In doing so we observe that many terms are the same within the square brackets of the first and second integrands, but there is a relative sign between the two integrals; hence those terms will cancel each other, and we get. ${ }^{13}$

$$
\begin{aligned}
\mathcal{L}_{3}^{\mu \nu}= & \frac{i}{2}(8 \pi G) \sum_{a, b=1}^{M+N} \int_{\omega}^{L^{-1}} \frac{d^{4} \ell}{(2 \pi)^{4}}\left\{G_{r}(-\ell)\right\}^{2} \frac{1}{p_{b} \cdot \ell-i \epsilon} \frac{1}{p_{a} \cdot \ell+i \epsilon} \\
& \times\left[2 p_{a} \cdot p_{b} p_{a}^{\mu} \ell^{\nu} k_{\alpha} J_{b}^{\alpha \beta} \ell_{\beta}+2 p_{b} \cdot k p_{a}^{\mu} \ell^{\nu} p_{a \alpha} J_{b}^{\alpha \beta} \ell_{\beta}+2 p_{a} \cdot p_{b} p_{a}^{\nu} \ell^{\mu} k_{\alpha} J_{b}^{\alpha \beta} \ell_{\beta}+2 p_{b} \cdot k p_{a}^{\nu} \ell^{\mu} p_{a \alpha} J_{b}^{\alpha \beta} \ell_{\beta}\right. \\
& -2 p_{a} \cdot k p_{a}^{\nu} \ell^{\mu} p_{b \alpha} J_{b}^{\alpha \beta} \ell_{\beta}+4 k \cdot \ell p_{a}^{\mu} p_{a}^{\nu} p_{b \alpha} J_{b}^{\alpha \beta} \ell_{\beta}+2 k \cdot \ell p_{a} \cdot p_{b} p_{a}^{\mu} J_{b}^{\nu \alpha} \ell_{\alpha}+2 k \cdot \ell p_{b}^{\nu} p_{a}^{\mu} p_{a \alpha} J_{b}^{\alpha \beta} \ell_{\beta} \\
& -2 k \cdot \ell p_{a}^{2} p_{b}^{\mu} J_{b}^{\nu \alpha} \ell_{\alpha}-2 k \cdot \ell p_{a}^{2} p_{b}^{\nu} J_{b}^{\mu \alpha} \ell_{\alpha}-2 k \cdot \ell p_{b}^{\mu} p_{a}^{\nu} p_{a \alpha} J_{b}^{\alpha \beta} \ell_{\beta}-2 k \cdot \ell p_{a} \cdot p_{b} p_{a}^{\nu} J_{b}^{\mu \alpha} \ell_{\alpha} \\
& -2 p_{a} \cdot k p_{a} \cdot p_{b} \ell^{\nu} J_{b}^{\mu \alpha} \ell_{\alpha}-2 p_{a} \cdot k p_{b}^{\mu} \ell^{\nu} p_{a \alpha} J_{b}^{\alpha \beta} \ell_{\beta}-2 p_{a} \cdot k p_{a} \cdot p_{b} \ell^{\mu} J_{b}^{\nu \alpha} \ell_{\alpha}-2 p_{b}^{\nu} \ell^{\mu} p_{a} \cdot k p_{a \alpha} J_{b}^{\alpha \beta} \ell_{\beta} \\
& \left.+p_{a}^{2} p_{b} \cdot k \ell^{\mu} J_{b}^{\nu \alpha} \ell_{\alpha}+p_{a}^{2} \ell^{\mu} p_{b}^{\nu} k_{\alpha} J_{b}^{\alpha \beta} \ell_{\beta}\right] .
\end{aligned}
$$

The last two lines in Eq. (3.53) represent $\mathcal{L}_{4}^{\mu \nu}$, which takes the following form after contracting the indices of the last line of Eq. (3.53) and removing the terms containing $p_{a} . \ell$ and $p_{b} . \ell$ in the numerator, as they give a vanishing contribution when

\footnotetext{
${ }^{13}$ Here also the same logic holds as described in footnote 12.
} 
we perform the $\ell$ integration,

$$
\begin{aligned}
\mathcal{L}_{4}^{\mu \nu}= & \frac{i}{2}(8 \pi G) \sum_{a, b=1}^{M+N} \int_{\omega}^{L^{-1}} \frac{d^{4} \ell}{(2 \pi)^{4}}\left\{G_{r}(-\ell)\right\}^{2} \frac{1}{p_{b} \cdot \ell-i \epsilon} \frac{1}{p_{a} \cdot \ell+i \epsilon} \\
& \times\left[2 \ell^{\mu} \ell^{\nu} p_{a} \cdot p_{b} p_{b \rho} J_{a}^{\rho \sigma} k_{\sigma}+p_{b}^{2} p_{a}^{\nu} \ell^{\mu} \ell_{\rho} J_{a}^{\rho \sigma} k_{\sigma}-p_{b}^{2} \ell^{\mu} \ell^{\nu} p_{a \rho} J_{a}^{\rho \sigma} k_{\sigma}+\frac{1}{2} p_{b}^{2} \ell^{2} \eta^{\mu \nu} p_{a \rho} J_{a}^{\rho \sigma} k_{\sigma}\right. \\
& -\ell^{2} p_{b}^{2} p_{a}^{\mu} J_{a}^{\nu \sigma} k_{\sigma}-\ell^{2} p_{b}^{2} p_{a}^{\nu} J_{a}^{\mu \sigma} k_{\sigma}+2 \ell^{2} p_{a} \cdot p_{b} p_{b}^{\mu} J_{a}^{\nu \sigma} k_{\sigma}+2 \ell^{2} p_{a}^{\nu} p_{b}^{\mu} p_{b \rho} J_{a}^{\rho \sigma} k_{\sigma} \\
& \left.-\ell^{2} p_{a} \cdot p_{b} \eta^{\mu \nu} p_{b \rho} J_{a}^{\rho \sigma} k_{\sigma}\right] .
\end{aligned}
$$

Let us first evaluate the sum of the contributions in Eqs. (B6) and (B9),

$$
\begin{aligned}
\mathcal{L}_{1}^{\mu \nu}+\mathcal{L}_{3}^{\mu \nu}= & \frac{i}{2}(8 \pi G) \sum_{a, b=1}^{M+N} \int_{\omega}^{L^{-1}} \frac{d^{4} \ell}{(2 \pi)^{4}}\left\{G_{r}(-\ell)\right\}^{2} \frac{1}{p_{b} \cdot \ell-i \epsilon} \frac{1}{p_{a} \cdot \ell+i \epsilon} \\
& \times\left[2 k \cdot \ell p_{b}^{\mu} p_{a}^{\nu} p_{a \alpha} J_{b}^{\alpha \beta} \ell_{\beta}-2 k \cdot \ell p_{b}^{\nu} p_{a}^{\mu} p_{a \rho} J_{b}^{\rho \sigma} \ell_{\sigma}+2 p_{a} \cdot p_{b} p_{a}^{\mu} \ell^{\nu} k_{\alpha} J_{b}^{\alpha \beta} \ell_{\beta}+2 p_{b} \cdot k p_{a}^{\mu} \ell^{\nu} p_{a \alpha} J_{b}^{\alpha \beta} \ell_{\beta}\right. \\
& +2 p_{a} \cdot p_{b} p_{a}^{\nu} \ell^{\mu} k_{\alpha} J_{b}^{\alpha \beta} \ell_{\beta}+2 p_{b} \cdot k p_{a}^{\nu} \ell^{\mu} p_{a \alpha} J_{b}^{\alpha \beta} \ell_{\beta}-2 p_{a} \cdot k p_{a}^{\nu} \ell^{\mu} p_{b \alpha} J_{b}^{\alpha \beta} \ell_{\beta}-2 k \cdot \ell p_{a} \cdot p_{b} p_{a}^{\mu} J_{b}^{\nu \alpha} \ell_{\alpha} \\
& +2 k \cdot \ell p_{a} \cdot p_{b} p_{a}^{\nu} J_{b}^{\mu \alpha} \ell_{\alpha}-2 p_{a} \cdot k p_{a} \cdot p_{b} \ell^{\nu} J_{b}^{\mu \alpha} \ell_{\alpha}-2 p_{a} \cdot k p_{b}^{\mu} \ell^{\nu} p_{a \alpha} J_{b}^{\alpha \beta} \ell_{\beta}-2 p_{a} \cdot k p_{a} \cdot p_{b} \ell^{\mu} J_{b}^{\nu \alpha} \ell_{\alpha} \\
& \left.-2 p_{b}^{\nu} \ell^{\mu} p_{a} \cdot k p_{a \alpha} J_{b}^{\alpha \beta} \ell_{\beta}+p_{a}^{2} p_{b} \cdot k \ell^{\mu} J_{b}^{\nu \alpha} \ell_{\alpha}+p_{a}^{2} \ell^{\mu} p_{b}^{\nu} k_{\alpha} J_{b}^{\alpha \beta} \ell_{\beta}\right] .
\end{aligned}
$$

Now using the result of the integral given in Eq. (B1) we get

$$
\begin{aligned}
& \mathcal{L}_{1}^{\mu \nu}+\mathcal{L}_{3}^{\mu \nu}=\frac{i G}{2} \sum_{\substack{a, b=1 \\
\text { aba } \\
\eta a \eta_{b}=1}}^{M+N} \frac{1}{\left[\left(p_{a} \cdot p_{b}\right)^{2}-p_{a}^{2} p_{b}^{2}\right]^{3 / 2}} \ln \left\{L\left(\omega+i \epsilon \eta_{a}\right)\right\} \\
& \times\left[2 p_{a} \cdot p_{b} p_{a} \cdot k p_{b}^{\mu} p_{a}^{\nu} p_{a \alpha} J_{b}^{\alpha \beta} p_{b \beta}-4 p_{a} \cdot p_{b} p_{a} \cdot k p_{b}^{\nu} p_{a}^{\mu} p_{a \alpha} J_{b}^{\alpha \beta} p_{b \beta}\right. \\
& -4 p_{a}^{2} p_{b} \cdot k p_{b}^{\mu} p_{a}^{\nu} p_{a \alpha} J_{b}^{\alpha \beta} p_{b \beta}-4\left(p_{a} \cdot p_{b}\right)^{2} p_{b}^{\mu} p_{a}^{\nu} p_{a \alpha} J_{b}^{\alpha \beta} k_{\beta}+2 p_{a}^{2} p_{b}^{2} p_{b}^{\mu} p_{a}^{\nu} p_{a \alpha} J_{b}^{\alpha \beta} k_{\beta} \\
& -p_{a}^{2} p_{b}^{2} p_{a}^{\mu} p_{b}^{\nu} p_{a \alpha} J_{b}^{\alpha \beta} k_{\beta}-4\left(p_{a} \cdot p_{b}\right)^{2} p_{a}^{\mu} p_{a}^{\nu} p_{b \alpha} J_{b}^{\alpha \beta} k_{\beta}+4 p_{a} \cdot p_{b} p_{b}^{2} p_{a}^{\mu} p_{a}^{\nu} p_{a \alpha} J_{b}^{\alpha \beta} k_{\beta} \\
& +p_{a} \cdot p_{b} p_{a}^{2} p_{a}^{\mu} p_{b}^{\nu} p_{b \alpha} J_{b}^{\alpha \beta} k_{\beta}+2 p_{a} \cdot p_{b} p_{a}^{2} p_{b}^{\mu} p_{a}^{\nu} p_{b \alpha} J_{b}^{\alpha \beta} k_{\beta}+4\left(p_{a} \cdot p_{b}\right)^{2} p_{a} \cdot k p_{a}^{\mu} p_{b \alpha} J_{b}^{\alpha \nu} \\
& +4 p_{a} \cdot p_{b} p_{b} \cdot k p_{a}^{\mu} p_{a}^{\nu} p_{a \alpha} J_{b}^{\alpha \beta} p_{b \beta}+3 p_{b} \cdot k p_{a}^{2} p_{b}^{2} p_{a}^{\mu} p_{a \alpha} J_{b}^{\alpha \nu}-4 p_{b} \cdot k\left(p_{a} \cdot p_{b}\right)^{2} p_{a}^{\nu} p_{a \alpha} J_{b}^{\alpha \mu} \\
& +2 p_{b} \cdot k p_{a}^{2} p_{b}^{2} p_{a}^{\nu} p_{a \alpha} J_{b}^{\alpha \mu}+2 p_{a} \cdot k p_{b}^{2} p_{a}^{\mu} p_{a}^{\nu} p_{b \alpha} J_{b}^{\alpha \beta} p_{a \beta}-3 p_{a}^{2} p_{a} \cdot p_{b} p_{b} \cdot k p_{a}^{\mu} p_{b \alpha} J_{b}^{\alpha \nu} \\
& +2 p_{a}^{2} p_{a} \cdot p_{b} p_{b} \cdot k p_{a}^{\nu} p_{b \alpha} J_{b}^{\alpha \mu}-4 p_{a} \cdot p_{b} p_{b}^{2} p_{a} \cdot k p_{a}^{\mu} p_{a \alpha} J_{b}^{\alpha \nu}+4 p_{a} \cdot p_{b}\left\{\left(p_{a} \cdot p_{b}\right)^{2}-p_{a}^{2} p_{b}^{2}\right\} p_{a}^{\mu} J_{b}^{\nu \alpha} k_{\alpha} \\
& +4 p_{a} \cdot k\left(p_{a} \cdot p_{b}\right)^{2} p_{b}^{\nu} p_{a \alpha} J_{b}^{\alpha \mu}-2 p_{a} \cdot k p_{a}^{2} p_{b}^{2} p_{b}^{\nu} p_{a \alpha} J_{b}^{\alpha \mu}-2 p_{a} \cdot k p_{a} \cdot p_{b} p_{a}^{2} p_{b}^{\nu} p_{b \alpha} J_{b}^{\alpha \mu} \\
& +4 p_{a} \cdot k\left(p_{a} \cdot p_{b}\right)^{2} p_{b}^{\mu} p_{a \alpha} J_{b}^{\alpha \nu}-2 p_{a} \cdot k p_{a}^{2} p_{b}^{2} p_{b}^{\mu} p_{a \alpha} J_{b}^{\alpha \nu}-2 p_{a} \cdot k p_{a} \cdot p_{b} p_{a}^{2} p_{b}^{\mu} p_{b \alpha} J_{b}^{\alpha \nu} \\
& +4 p_{a} \cdot k p_{a}^{2} p_{b}^{\mu} p_{b}^{\nu} p_{a \alpha} J_{b}^{\alpha \beta} p_{b \beta}-p_{a}^{2} p_{a} \cdot p_{b} p_{b} \cdot k p_{b}^{\mu} p_{a \alpha} J_{b}^{\alpha \nu}+\left(p_{a}^{2}\right)^{2} p_{b} \cdot k p_{b}^{\mu} p_{b \alpha} J_{b}^{\alpha \nu} \\
& +p_{a}^{2} p_{b} \cdot k\left\{\left(p_{a} \cdot p_{b}\right)^{2}-p_{a}^{2} p_{b}^{2}\right\} J_{b}^{\mu \nu}+p_{a}^{2}\left\{\left(p_{a} \cdot p_{b}\right)^{2}-p_{a}^{2} p_{b}^{2}\right\} p_{b}^{\nu} J_{b}^{\mu \rho} k_{\rho} \\
& \left.-p_{a}^{2} p_{a} \cdot p_{b} p_{b}^{\mu} p_{b}^{\nu} p_{a \alpha} J_{b}^{\alpha \beta} k_{\beta}+\left(p_{a}^{2}\right)^{2} p_{b}^{\mu} p_{b}^{\nu} p_{b \alpha} J_{b}^{\alpha \beta} k_{\beta}+2 p_{a} \cdot k\left\{\left(p_{a} \cdot p_{b}\right)^{2}-p_{a}^{2} p_{b}^{2}\right\} p_{a}^{\nu} p_{b \alpha} J_{b}^{\alpha \mu}\right] \text {. }
\end{aligned}
$$

In the above expression we substitute $J_{b}^{\mu \nu}=r_{b}^{\mu} p_{b}^{\nu}-r_{b}^{\nu} p_{b}^{\mu}+\Sigma_{b}^{\mu \nu}$ and simplify using SSC, $p_{b \mu} \Sigma_{b}^{\mu \nu}=0$. Since $a$ and $b$ are dummy indices, we interchange them and then the full expression can be written in terms of $r_{a}$ and $\Sigma_{a}$. After all these steps finally we get 


$$
\begin{aligned}
& \mathcal{L}_{1}^{\mu \nu}+\mathcal{L}_{3}^{\mu \nu}=\frac{i G}{2} \sum_{\substack{a b=1 \\
b \neq a \\
\eta^{\prime} \eta_{b}=1}}^{M+N} \frac{1}{\left[\left(p_{a} \cdot p_{b}\right)^{2}-p_{a}^{2} p_{b}^{2}\right]^{3 / 2}} \ln \left\{L\left(\omega+i \epsilon \eta_{a}\right)\right\} \\
& \times\left[2 p_{a} \cdot p_{b} p_{b} \cdot k p_{a}^{\mu} p_{b}^{\nu} p_{b} \cdot r_{a} p_{a}^{2}+8 p_{b}^{2} p_{a} \cdot k p_{a}^{\mu} p_{b}^{\nu} p_{a} \cdot p_{b} p_{a} \cdot r_{a}-8\left(p_{a} \cdot p_{b}\right)^{2} p_{a}^{\mu} p_{b}^{\nu} p_{b} \cdot r_{a} p_{a} \cdot k\right. \\
& +4\left(p_{a} \cdot p_{b}\right)^{3} p_{a}^{\mu} p_{b}^{\nu} r_{a} \cdot k-4 p_{a}^{2} p_{b}^{2} p_{a}^{\mu} p_{b}^{\nu} p_{a} \cdot p_{b} r_{a} \cdot k \\
& -2 p_{a}^{2} p_{b}^{2} p_{b} \cdot k p_{b}^{\nu} p_{a}^{\mu} p_{a} \cdot r_{a}-8 p_{a} \cdot p_{b} p_{b} \cdot k p_{a}^{\nu} p_{b}^{\mu} p_{b} \cdot r_{a} p_{a}^{2}+8\left(p_{a} \cdot p_{b}\right)^{2} p_{b} \cdot k p_{a}^{\nu} p_{b}^{\mu} p_{a} \cdot r_{a} \\
& +2 p_{a}^{2} p_{b}^{2} p_{b}^{\mu} p_{a}^{\nu} p_{b} \cdot r_{a} p_{a} \cdot k+4 p_{a}^{2} p_{b}^{2} p_{b}^{\mu} p_{a}^{\nu} p_{a} \cdot p_{b} r_{a} \cdot k-2 p_{a} \cdot p_{b} p_{b}^{2} p_{b}^{\mu} p_{a}^{\nu} p_{a} \cdot r_{a} p_{a} \cdot k \\
& -4\left(p_{a} \cdot p_{b}\right)^{3} p_{b}^{\mu} p_{a}^{\nu} r_{a} \cdot k-8\left(p_{a} \cdot p_{b}\right)^{2} p_{b}^{\mu} p_{b}^{\nu} p_{a} \cdot r_{a} p_{a} \cdot k+8 p_{a} \cdot p_{b} p_{a}^{2} p_{b}^{\mu} p_{b}^{\nu} p_{b} \cdot r_{a} p_{a} \cdot k \\
& +2 p_{b} \cdot k p_{a}^{2} p_{b}^{\mu} p_{b}^{\nu} p_{a} \cdot r_{a} p_{a} \cdot p_{b}-2 p_{b} \cdot k\left(p_{a}^{2}\right)^{2} p_{b}^{\mu} p_{b}^{\nu} p_{b} \cdot r_{a}+4 p_{a} \cdot p_{b}\left\{\left(p_{a} \cdot p_{b}\right)^{2}-p_{a}^{2} p_{b}^{2}\right\} p_{a} \cdot k p_{b}^{\mu} r_{a}^{\nu} \\
& +4 p_{a} \cdot k\left(p_{a} \cdot p_{b}\right)^{3} p_{b}^{\nu} r_{a}^{\mu}-4 p_{a} \cdot k p_{a}^{2} p_{b}^{2} p_{a} \cdot p_{b} p_{b}^{\nu} r_{a}^{\mu}-2 p_{b} \cdot k\left\{\left(p_{a} \cdot p_{b}\right)^{2}-p_{a}^{2} p_{b}^{2}\right\} p_{a}^{2} p_{b}^{\nu} r_{a}^{\mu} \\
& +8 p_{b} \cdot k\left(p_{a} \cdot p_{b}\right)^{2} p_{a}^{\nu} p_{b} \cdot r_{a} p_{a}^{\mu}-8 p_{b} \cdot k p_{a} \cdot p_{b} p_{b}^{2} p_{a} \cdot r_{a} p_{a}^{\mu} p_{a}^{\nu}-2 p_{b}^{2} p_{a} \cdot p_{b} p_{a} \cdot k p_{a}^{\mu} p_{a}^{\nu} p_{b} \cdot r_{a} \\
& +2\left(p_{b}^{2}\right)^{2} p_{a} \cdot k p_{a} \cdot r_{a} p_{a}^{\mu} p_{a}^{\nu}-4 p_{b} \cdot k\left(p_{a} \cdot p_{b}\right)^{3} p_{a}^{\nu} r_{a}^{\mu}+4 p_{b} \cdot k p_{a}^{2} p_{b}^{2} p_{a} \cdot p_{b} p_{a}^{\nu} r_{a}^{\mu} \\
& +p_{b}^{2}\left\{\left(p_{a} \cdot p_{b}\right)^{2}-p_{a}^{2} p_{b}^{2}\right\} p_{a} \cdot k p_{a}^{\nu} r_{a}^{\mu}-4 p_{b} \cdot k\left(p_{a} \cdot p_{b}\right)^{3} p_{a}^{\mu} r_{a}^{\nu}+4 p_{b} \cdot k p_{a}^{2} p_{b}^{2} p_{a} \cdot p_{b} p_{a}^{\mu} r_{a}^{\nu} \\
& +p_{b}^{2}\left\{\left(p_{a} \cdot p_{b}\right)^{2}-p_{a}^{2} p_{b}^{2}\right\} p_{a} \cdot k p_{a}^{\mu} r_{a}^{\nu}-4\left(p_{a} \cdot p_{b}\right)^{2} p_{a}^{\mu} p_{b}^{\nu} p_{b \alpha} \Sigma_{a}^{\alpha \beta} k_{\beta}+2 p_{a}^{2} p_{b}^{2} p_{a}^{\mu} p_{b}^{\nu} p_{b \alpha} \Sigma_{a}^{\alpha \beta} k_{\beta} \\
& -p_{a}^{2} p_{b}^{2} p_{b}^{\mu} p_{a}^{\nu} p_{b \alpha} \Sigma_{a}^{\alpha \beta} k_{\beta}+4 p_{a} \cdot p_{b} p_{a}^{2} p_{b}^{\mu} p_{b}^{\nu} p_{b \alpha} \Sigma_{a}^{\alpha \beta} k_{\beta}+3 p_{a} \cdot k p_{a}^{2} p_{b}^{2} p_{b}^{\mu} p_{b \alpha} \Sigma_{a}^{\alpha \nu} \\
& -4 p_{a} \cdot k\left(p_{a} \cdot p_{b}\right)^{2} p_{b}^{\nu} p_{b \alpha} \Sigma_{a}^{\alpha \mu}+2 p_{a} \cdot k p_{a}^{2} p_{b}^{2} p_{b}^{\nu} p_{b \alpha} \Sigma_{a}^{\alpha \mu}-4 p_{a} \cdot p_{b} p_{a}^{2} p_{b} \cdot k p_{b}^{\mu} p_{b \alpha} \Sigma_{a}^{\alpha \nu} \\
& +4 p_{a} \cdot p_{b}\left\{\left(p_{a} \cdot p_{b}\right)^{2}-p_{a}^{2} p_{b}^{2}\right\} p_{b}^{\mu} \Sigma_{a}^{\nu \alpha} k_{\alpha}+4 p_{b} \cdot k\left(p_{a} \cdot p_{b}\right)^{2} p_{a}^{\nu} p_{b \alpha} \Sigma_{a}^{\alpha \mu}-2 p_{b} \cdot k p_{a}^{2} p_{b}^{2} p_{a}^{\nu} p_{b \alpha} \Sigma_{a}^{\alpha \mu} \\
& +4 p_{b} \cdot k\left(p_{a} \cdot p_{b}\right)^{2} p_{a}^{\mu} p_{b \alpha} \Sigma_{a}^{\alpha \nu}-2 p_{b} \cdot k p_{a}^{2} p_{b}^{2} p_{a}^{\mu} p_{b \alpha} \Sigma_{a}^{\alpha \nu}-p_{b}^{2} p_{a} \cdot p_{b} p_{a} \cdot k p_{a}^{\mu} p_{b \alpha} \Sigma_{a}^{\alpha \nu} \\
& \left.+p_{b}^{2} p_{a} \cdot k\left\{\left(p_{a} \cdot p_{b}\right)^{2}-p_{a}^{2} p_{b}^{2}\right\} J_{a}^{\mu \nu}+p_{b}^{2}\left\{\left(p_{a} \cdot p_{b}\right)^{2}-p_{a}^{2} p_{b}^{2}\right\} p_{a}^{\nu} \Sigma_{a}^{\mu \rho} k_{\rho}-p_{b}^{2} p_{a} \cdot p_{b} p_{a}^{\mu} p_{a}^{\nu} p_{b \alpha} \Sigma_{a}^{\alpha \beta} k_{\beta}\right] .
\end{aligned}
$$

From Eq. (B10) using the result of the integration (B1), we get

$$
\begin{aligned}
\mathcal{L}_{4}^{\mu \nu}= & \frac{i G}{2} \sum_{\substack{a, b=1 \\
b \neq a \\
\eta_{a} \eta_{b}=1}}^{M+N} \frac{1}{\left[\left(p_{a} \cdot p_{b}\right)^{2}-p_{a}^{2} p_{b}^{2}\right]^{3 / 2}} \ln \left\{L\left(\omega+i \epsilon \eta_{a}\right)\right\}\left[2\left(p_{a} \cdot p_{b}\right)^{2} p_{b \rho} J_{a}^{\rho \sigma} k_{\sigma}\left(p_{a}^{\mu} p_{b}^{\nu}+p_{a}^{\nu} p_{b}^{\mu}\right)\right. \\
& -2 p_{a} \cdot p_{b} p_{a}^{2} p_{b \rho} J_{a}^{\rho \sigma} k_{\sigma} p_{b}^{\mu} p_{b}^{\nu}-p_{a} \cdot p_{b} p_{b}^{2} p_{b \rho} J_{a}^{\rho \sigma} k_{\sigma} p_{a}^{\mu} p_{a}^{\nu}-p_{a}^{2} p_{b}^{2} p_{b \rho} J_{a}^{\rho \sigma} k_{\sigma} p_{a}^{\nu} p_{b}^{\mu} \\
& \left.-p_{a} \cdot p_{b} p_{b}^{2} p_{a \rho} J_{a}^{\rho \sigma} k_{\sigma} p_{a}^{\mu} p_{b}^{\nu}+p_{a}^{2} p_{b}^{2} p_{a \rho} J_{a}^{\rho \sigma} k_{\sigma} p_{b}^{\mu} p_{b}^{\nu}\right]-(i G) \sum_{\substack{a, b=1 \\
b \neq a \\
\eta_{\eta} \eta_{b}=1}}^{M+N} \frac{1}{\left[\left(p_{a} \cdot p_{b}\right)^{2}-p_{a}^{2} p_{b}^{2}\right]^{1 / 2}} \ln \left\{L\left(\omega+i \epsilon \eta_{a}\right)\right\} \\
& \times\left[-p_{b}^{2} p_{a}^{\mu} J_{a}^{\nu \alpha} k_{\alpha}-\frac{1}{2} p_{b}^{2} p_{a}^{\nu} J_{a}^{\mu \alpha} k_{\alpha}+2 p_{a} \cdot p_{b} p_{b}^{\mu} J_{a}^{\nu \alpha} k_{\alpha}+2 p_{a}^{\nu} p_{b}^{\mu} p_{b \rho} J_{a}^{\rho \sigma} k_{\sigma}\right]
\end{aligned}
$$

Now substituting $J_{a}^{\mu \nu}=r_{a}^{\mu} p_{a}^{\nu}-r_{a}^{\nu} p_{a}^{\mu}+\Sigma_{a}^{\mu \nu}$ in the above expression and using SSC: $p_{a \mu} \Sigma_{a}^{\mu \nu}=0$, we get

$$
\begin{aligned}
\mathcal{L}_{4}^{\mu \nu}= & \frac{i G}{2} \sum_{\substack{a, b=1 \\
b \neq a \\
\eta_{a} \eta_{b}=1}}^{M+N} \frac{1}{\left[\left(p_{a} \cdot p_{b}\right)^{2}-p_{a}^{2} p_{b}^{2}\right]^{3 / 2}} \ln \left\{L\left(\omega+i \epsilon \eta_{a}\right)\right\}\left[2\left(p_{a} \cdot p_{b}\right)^{2} p_{b} \cdot r_{a} p_{a} \cdot k\left(p_{a}^{\mu} p_{b}^{\nu}+p_{a}^{\nu} p_{b}^{\mu}\right)\right. \\
& -2\left(p_{a} \cdot p_{b}\right)^{3} r_{a} \cdot k\left(p_{a}^{\mu} p_{b}^{\nu}+p_{a}^{\nu} p_{b}^{\mu}\right)-2 p_{a} \cdot p_{b} p_{a}^{2} p_{b} \cdot r_{a} p_{a} \cdot k p_{b}^{\mu} p_{b}^{\nu}+2\left(p_{a} \cdot p_{b}\right)^{2} p_{a}^{2} r_{a} \cdot k p_{b}^{\mu} p_{b}^{\nu} \\
& -p_{a} \cdot p_{b} p_{b}^{2} p_{b} \cdot r_{a} p_{a} \cdot k p_{a}^{\mu} p_{a}^{\nu}+\left(p_{a} \cdot p_{b}\right)^{2} p_{b}^{2} r_{a} \cdot k p_{a}^{\mu} p_{a}^{\nu}-p_{a}^{2} p_{b}^{2} p_{b} \cdot r_{a} p_{a} \cdot k p_{a}^{\nu} p_{b}^{\mu}+p_{a}^{2} p_{b}^{2} p_{a} \cdot p_{b} r_{a} \cdot k p_{a}^{\nu} p_{b}^{\mu} \\
& -p_{a} \cdot p_{b} p_{b}^{2} p_{a} \cdot r_{a} p_{a} \cdot k p_{a}^{\mu} p_{b}^{\nu}+p_{a} \cdot p_{b} p_{a}^{2} p_{b}^{2} r_{a} \cdot k p_{a}^{\mu} p_{b}^{\nu}+p_{a}^{2} p_{b}^{2} p_{a} \cdot r_{a} p_{a} \cdot k p_{b}^{\mu} p_{b}^{\nu}-\left(p_{a}^{2}\right)^{2} p_{b}^{2} r_{a} \cdot k p_{b}^{\mu} p_{b}^{\nu} \\
& +2\left(p_{a} \cdot p_{b}\right)^{2} p_{b \rho} \Sigma_{a}^{\rho \sigma} k_{\sigma}\left(p_{a}^{\mu} p_{b}^{\nu}+p_{a}^{\nu} p_{b}^{\mu}\right)-2 p_{a} \cdot p_{b} p_{a}^{2} p_{b \rho} \Sigma_{a}^{\rho \sigma} k_{\sigma} p_{b}^{\mu} p_{b}^{\nu}-p_{a} \cdot p_{b} p_{b}^{2} p_{b \rho} \Sigma_{a}^{\rho \sigma} k_{\sigma} p_{a}^{\mu} p_{a}^{\nu} \\
& \left.-p_{a}^{2} p_{b}^{2} p_{b \rho} \Sigma_{a}^{\rho \sigma} k_{\sigma} p_{a}^{\nu} p_{b}^{\mu}\right]
\end{aligned}
$$




$$
\begin{aligned}
& -(i G) \sum_{\substack{a, b=1 \\
b \neq a \\
\eta_{a} \eta_{b}=1}}^{M+N} \frac{1}{\left[\left(p_{a} \cdot p_{b}\right)^{2}-p_{a}^{2} p_{b}^{2}\right]^{1 / 2}} \ln \left\{L\left(\omega+i \epsilon \eta_{a}\right)\right\}\left[-p_{b}^{2} p_{a}^{\mu} r_{a}^{\nu} p_{a} \cdot k+\frac{3}{2} p_{b}^{2} p_{a}^{\mu} p_{a}^{\nu} r_{a} \cdot k\right. \\
& -\frac{1}{2} p_{b}^{2} p_{a}^{\nu} r_{a}^{\mu} p_{a} \cdot k+2 p_{a} \cdot p_{b} p_{b}^{\mu} r_{a}^{\nu} p_{a} \cdot k-4 p_{a} \cdot p_{b} p_{b}^{\mu} p_{a}^{\nu} r_{a} \cdot k+2 p_{a}^{\nu} p_{b}^{\mu} p_{b} \cdot r_{a} p_{a} \cdot k \\
& \left.-p_{b}^{2} p_{a}^{\mu} \Sigma_{a}^{\nu \alpha} k_{\alpha}-\frac{1}{2} p_{b}^{2} p_{a}^{\nu} \Sigma_{a}^{\mu \alpha} k_{\alpha}+2 p_{a} \cdot p_{b} p_{b}^{\mu} \Sigma_{a}^{\nu \alpha} k_{\alpha}+2 p_{a}^{\nu} p_{b}^{\mu} p_{b \rho} \Sigma_{a}^{\rho \sigma} k_{\sigma}\right] .
\end{aligned}
$$

The contribution from Eq. (B7) after using the results of integrations (B1), (B2), and (B3) takes the following form:

$$
\begin{aligned}
& \mathcal{L}_{2}^{\mu \nu}=\frac{i G}{2} \sum_{\substack{a, b=1 \\
b \neq a \\
\eta_{a} \eta_{b}=1}}^{M+N} \frac{p_{a} \cdot k}{\left[\left(p_{a} \cdot p_{b}\right)^{2}-p_{a}^{2} p_{b}^{2}\right]^{5 / 2}} \ln \left\{L\left(\omega+i \epsilon \eta_{a}\right)\right\} \\
& \times\left[6\left(p_{a} \cdot p_{b}\right)^{3} p_{a}^{\mu} p_{b}^{\nu} p_{a \alpha} J_{b}^{\alpha \beta} p_{b \beta}-3\left(p_{a} \cdot p_{b}\right)^{2} p_{a}^{2} p_{b}^{\mu} p_{b}^{\nu} p_{a \alpha} J_{b}^{\alpha \beta} p_{b \beta}-6\left(p_{a} \cdot p_{b}\right)^{2} p_{b}^{2} p_{a}^{\mu} p_{a}^{\nu} p_{a \alpha} J_{b}^{\alpha \beta} p_{b \beta}\right. \\
& \left.+3 p_{a} \cdot p_{b} p_{a}^{2} p_{b}^{2} p_{b}^{\mu} p_{a}^{\nu} p_{a \alpha} J_{b}^{\alpha \beta} p_{b \beta}-3 p_{a} \cdot p_{b} p_{a}^{2} p_{b}^{2} p_{a}^{\mu} p_{b}^{\nu} p_{a \alpha} J_{b}^{\alpha \beta} p_{b \beta}+3 p_{a}^{2}\left(p_{b}^{2}\right)^{2} p_{a}^{\mu} p_{a}^{\nu} p_{a \alpha} J_{b}^{\alpha \beta} p_{b \beta}\right] \\
& -\frac{i G}{2} \sum_{\substack{a, b=1 \\
b=a \\
\eta_{a} \eta_{b}=1}}^{M+N} \frac{p_{a} \cdot k}{\left[\left(p_{a} \cdot p_{b}\right)^{2}-p_{a}^{2} p_{b}^{2}\right]^{5 / 2}} \ln \left\{L\left(\omega+i \epsilon \eta_{a}\right)\right\} \\
& \times\left[3 p_{b}^{2}\left(p_{a} \cdot p_{b}\right)^{2} p_{a}^{\mu} p_{b}^{\nu} p_{a \alpha} J_{a}^{\alpha \beta} p_{b \beta}+3 p_{a}^{2} p_{b}^{2} p_{a} \cdot p_{b} p_{b}^{\mu} p_{b}^{\nu} p_{a \alpha} J_{a}^{\alpha \beta} p_{b \beta}-3\left(p_{b}^{2}\right)^{2} p_{a} \cdot p_{b} p_{a}^{\mu} p_{a}^{\nu} p_{a \alpha} J_{a}^{\alpha \beta} p_{b \beta}\right. \\
& \left.-3\left(p_{b}^{2}\right)^{2} p_{a}^{2} p_{b}^{\mu} p_{a}^{\nu} p_{a \alpha} J_{a}^{\alpha \beta} p_{b \beta}+6\left(p_{a} \cdot p_{b}\right)^{3} p_{b}^{\mu} p_{b}^{\nu} p_{b \alpha} J_{a}^{\alpha \beta} p_{a \beta}-6\left(p_{a} \cdot p_{b}\right)^{2} p_{b}^{2} p_{b}^{\mu} p_{a}^{\nu} p_{b \alpha} J_{a}^{\alpha \beta} p_{a \beta}\right] \\
& -\frac{i G}{2} \sum_{\substack{a, b=1 \\
b=a \\
\eta_{a} \eta_{b}=1}}^{M+N} \frac{p_{a} \cdot k}{\left[\left(p_{a} \cdot p_{b}\right)^{2}-p_{a}^{2} p_{b}^{2}\right]^{3 / 2}} \ln \left\{L\left(\omega+i \epsilon \eta_{a}\right)\right\}\left[2 p_{a} \cdot p_{b} p_{a}^{\mu} p_{b}^{\nu} p_{a \alpha} J_{b}^{\alpha \beta} p_{b \beta}\right. \\
& +p_{a}^{2} p_{b}^{\mu} p_{b}^{\nu} p_{a \alpha} J_{b}^{\alpha \beta} p_{b \beta}+2\left(p_{a} \cdot p_{b}\right)^{2} p_{b}^{\nu} p_{a \alpha} J_{b}^{\alpha \mu}-p_{a}^{2} p_{a} \cdot p_{b} p_{b}^{\nu} p_{b \alpha} J_{b}^{\alpha \mu}+2 p_{a} \cdot p_{b} p_{b}^{2} p_{a}^{\nu} p_{a \alpha} J_{b}^{\alpha \mu} \\
& -p_{a}^{2} p_{b}^{2} p_{a}^{\nu} p_{b \alpha} J_{b}^{\alpha \mu}+2\left(p_{a} \cdot p_{b}\right)^{2} p_{b}^{\mu} p_{a \alpha} J_{b}^{\alpha \nu}-p_{a}^{2} p_{a} \cdot p_{b} p_{b}^{\mu} p_{b \alpha} J_{b}^{\alpha \nu}-2 p_{a} \cdot p_{b} p_{b}^{2} p_{a}^{\mu} p_{a \alpha} J_{b}^{\alpha \nu} \\
& \left.+p_{a}^{2} p_{b}^{2} p_{a}^{\mu} p_{b \alpha} J_{b}^{\alpha \nu}-2\left(p_{a} \cdot p_{b}\right)^{2} p_{a}^{\nu} p_{b \alpha} J_{b}^{\alpha \mu}-6 p_{b}^{2} p_{a}^{\mu} p_{a}^{\nu} p_{a \alpha} J_{b}^{\alpha \beta} p_{b \beta}+2 p_{a} \cdot p_{b} p_{b}^{\mu} p_{a}^{\nu} p_{a \alpha} J_{b}^{\alpha \beta} p_{b \beta}\right] \\
& +\frac{i G}{2} \sum_{\substack{a, b=1 \\
b \neq a \\
\eta_{a} \eta_{b}=1}}^{M+N} \frac{p_{a} \cdot k}{\left[\left(p_{a} \cdot p_{b}\right)^{2}-p_{a}^{2} p_{b}^{2}\right]^{3 / 2}} \ln \left\{L\left(\omega+i \epsilon \eta_{a}\right)\right\}\left[-p_{b}^{2} p_{a}^{\mu} p_{b}^{\nu} p_{a \alpha} J_{a}^{\alpha \beta} p_{b \beta}\right. \\
& +6 p_{b}^{2} p_{b}^{\mu} p_{a}^{\nu} p_{a \alpha} J_{a}^{\alpha \beta} p_{b \beta}+2 p_{a} \cdot p_{b} p_{b}^{\mu} p_{b}^{\nu} p_{b \alpha} J_{a}^{\alpha \beta} p_{a \beta}+2\left(p_{a} \cdot p_{b}\right)^{2} p_{b}^{\nu} p_{b \alpha} J_{a}^{\alpha \mu}-p_{b}^{2} p_{a} \cdot p_{b} p_{b}^{\nu} p_{a \alpha} J_{a}^{\alpha \mu} \\
& -\left(p_{b}^{2}\right)^{2} p_{a}^{\nu} p_{a \alpha} J_{a}^{\alpha \mu}-2\left(p_{a} \cdot p_{b}\right)^{2} p_{b}^{\mu} p_{b \alpha} J_{a}^{\alpha \nu}+4 p_{b}^{2} p_{a} \cdot p_{b} p_{b}^{\mu} p_{a \alpha} J_{a}^{\alpha \nu}-2\left(p_{b}^{2}\right)^{2} p_{a}^{\mu} p_{a \alpha} J_{a}^{\alpha \nu} \\
& \left.+p_{b}^{2} p_{a} \cdot p_{b} p_{a}^{\mu} p_{b \alpha} J_{a}^{\alpha \nu}-p_{a}^{2} p_{b}^{2} p_{b}^{\mu} p_{b \alpha} J_{a}^{\alpha \nu}-p_{b}^{2}\left\{\left(p_{a} \cdot p_{b}\right)^{2}-p_{a}^{2} p_{b}^{2}\right\} J_{a}^{\mu \nu}\right] \text {. }
\end{aligned}
$$

Now in the above expression we substitute total angular momenta in terms of orbital and spin and interchange $a \leftrightarrow b$ in appropriate places to make the result $r_{a}$ and $\Sigma_{a}$ dependent. Then the above expression finally reduces to 


$$
\begin{aligned}
& \mathcal{L}_{2}^{\mu \nu}=\frac{i G}{2} \sum_{\substack{a, b=1 \\
b \neq a \\
\eta_{a} \eta_{b}=1}}^{M+N} \frac{1}{\left[\left(p_{a} \cdot p_{b}\right)^{2}-p_{a}^{2} p_{b}^{2}\right]^{5 / 2}} \ln \left\{L\left(\omega+i \epsilon \eta_{a}\right)\right\} \\
& \times\left[p _ { b } \cdot k \left\{6\left(p_{a} \cdot p_{b}\right)^{3} p_{b}^{\mu} p_{a}^{\nu} p_{b} \cdot r_{a} p_{a}^{2}-6\left(p_{a} \cdot p_{b}\right)^{4} p_{b}^{\mu} p_{a}^{\nu} p_{a} \cdot r_{a}-3\left(p_{a} \cdot p_{b}\right)^{2} p_{a}^{2} p_{b}^{2} p_{a}^{\mu} p_{a}^{\nu} p_{b} \cdot r_{a}\right.\right. \\
& +3\left(p_{a} \cdot p_{b}\right)^{3} p_{b}^{2} p_{a}^{\mu} p_{a}^{\nu} p_{a} \cdot r_{a}-6\left(p_{a} \cdot p_{b}\right)^{2}\left(p_{a}^{2}\right)^{2} p_{b}^{\mu} p_{b}^{\nu} p_{b} \cdot r_{a}+6\left(p_{a} \cdot p_{b}\right)^{3} p_{a}^{2} p_{b}^{\mu} p_{b}^{\nu} p_{a} \cdot r_{a} \\
& +3 p_{a} \cdot p_{b}\left(p_{a}^{2}\right)^{2} p_{b}^{2} p_{a}^{\mu} p_{b}^{\nu} p_{b} \cdot r_{a}-3\left(p_{a} \cdot p_{b}\right)^{2} p_{a}^{2} p_{b}^{2} p_{a}^{\mu} p_{b}^{\nu} p_{a} \cdot r_{a}-3 p_{a} \cdot p_{b}\left(p_{a}^{2}\right)^{2} p_{b}^{2} p_{b}^{\mu} p_{a}^{\nu} p_{b} \cdot r_{a} \\
& \left.+3\left(p_{a} \cdot p_{b}\right)^{2} p_{a}^{2} p_{b}^{2} p_{b}^{\mu} p_{a}^{\nu} p_{a} \cdot r_{a}+3 p_{b}^{2}\left(p_{a}^{2}\right)^{3} p_{b}^{\mu} p_{b}^{\nu} p_{b} \cdot r_{a}-3 p_{b}^{2}\left(p_{a}^{2}\right)^{2} p_{a} \cdot p_{b} p_{b}^{\mu} p_{b}^{\nu} p_{a} \cdot r_{a}\right\} \\
& -p_{a} \cdot k\left\{3 p_{b}^{2}\left(p_{a} \cdot p_{b}\right)^{3} p_{a}^{\mu} p_{b}^{\nu} p_{a} \cdot r_{a}-3 p_{b}^{2}\left(p_{a} \cdot p_{b}\right)^{2} p_{a}^{\mu} p_{b}^{\nu} p_{a}^{2} p_{b} \cdot r_{a}+3 p_{a}^{2} p_{b}^{2}\left(p_{a} \cdot p_{b}\right)^{2} p_{b}^{\mu} p_{b}^{\nu} p_{a} \cdot r_{a}\right. \\
& -3\left(p_{a}^{2}\right)^{2} p_{b}^{2} p_{a} \cdot p_{b} p_{b}^{\mu} p_{b}^{\nu} p_{b} \cdot r_{a}-3\left(p_{b}^{2}\right)^{2}\left(p_{a} \cdot p_{b}\right)^{2} p_{a}^{\mu} p_{a}^{\nu} p_{a} \cdot r_{a}+3\left(p_{b}^{2}\right)^{2} p_{a}^{2} p_{a} \cdot p_{b} p_{a}^{\mu} p_{a}^{\nu} p_{b} \cdot r_{a} \\
& -3\left(p_{b}^{2}\right)^{2} p_{a}^{2} p_{a} \cdot p_{b} p_{b}^{\mu} p_{a}^{\nu} p_{a} \cdot r_{a}+3\left(p_{a}^{2}\right)^{2}\left(p_{b}^{2}\right)^{2} p_{b}^{\mu} p_{a}^{\nu} p_{b} \cdot r_{a}+6\left(p_{a} \cdot p_{b}\right)^{3} p_{b}^{\mu} p_{b}^{\nu} p_{a}^{2} p_{b} \cdot r_{a} \\
& \left.\left.-6\left(p_{a} \cdot p_{b}\right)^{4} p_{b}^{\mu} p_{b}^{\nu} p_{a} \cdot r_{a}-6\left(p_{a} \cdot p_{b}\right)^{2} p_{a}^{2} p_{b}^{2} p_{b}^{\mu} p_{a}^{\nu} p_{b} \cdot r_{a}+6\left(p_{a} \cdot p_{b}\right)^{3} p_{b}^{2} p_{b}^{\mu} p_{a}^{\nu} p_{a} \cdot r_{a}\right\}\right] \\
& -\frac{i G}{2} \sum_{\substack{a, b=1 \\
b \neq a \\
\eta_{a} \eta_{b}=1}}^{M+N} \frac{1}{\left[\left(p_{a} \cdot p_{b}\right)^{2}-p_{a}^{2} p_{b}^{2}\right]^{3 / 2}} \ln \left\{L\left(\omega+i \epsilon \eta_{a}\right)\right\} \\
& \times\left[p _ { b } \cdot k \left\{-2\left(p_{a} \cdot p_{b}\right)^{2} p_{b}^{\mu} p_{a}^{\nu} p_{a} \cdot r_{a}+p_{a}^{2} p_{b}^{2} p_{a}^{\mu} p_{a}^{\nu} p_{b} \cdot r_{a}-3 p_{b}^{2} p_{a} \cdot p_{b} p_{a}^{\mu} p_{a}^{\nu} p_{a} \cdot r_{a}\right.\right. \\
& +4\left(p_{a} \cdot p_{b}\right)^{2} p_{a}^{\nu} p_{b} \cdot r_{a} p_{a}^{\mu}-2\left(p_{a} \cdot p_{b}\right)^{3} p_{a}^{\nu} r_{a}^{\mu}+p_{a}^{2} p_{b}^{2} p_{a} \cdot p_{b} p_{a}^{\nu} r_{a}^{\mu}+4 p_{a} \cdot p_{b} p_{a}^{2} p_{b}^{\nu} p_{b} \cdot r_{a} p_{a}^{\mu} \\
& -p_{a}^{2} p_{b}^{2} p_{b}^{\nu} p_{a} \cdot r_{a} p_{a}^{\mu}+\left(p_{a}^{2}\right)^{2} p_{b}^{2} p_{b}^{\nu} r_{a}^{\mu}-2\left(p_{a} \cdot p_{b}\right)^{3} p_{a}^{\mu} r_{a}^{\nu}+p_{a}^{2} p_{b}^{2} p_{a} \cdot p_{b} p_{a}^{\mu} r_{a}^{\nu} \\
& +2\left(p_{a} \cdot p_{b}\right)^{2} p_{a}^{2} p_{b}^{\mu} r_{a}^{\nu}+p_{a}^{2} p_{b}^{2} p_{b}^{\mu} p_{a} \cdot r_{a} p_{a}^{\nu}-\left(p_{a}^{2}\right)^{2} p_{b}^{2} p_{b}^{\mu} r_{a}^{\nu}-4\left(p_{a} \cdot p_{b}\right)^{2} p_{b}^{\nu} p_{a} \cdot r_{a} p_{a}^{\mu} \\
& -6\left(p_{a}^{2}\right)^{2} p_{b}^{\mu} p_{b}^{\nu} p_{b} \cdot r_{a}+6 p_{a}^{2} p_{b}^{\mu} p_{b}^{\nu} p_{a} \cdot p_{b} p_{a} \cdot r_{a}+2\left(p_{a} \cdot p_{b}\right)^{2} p_{a}^{\nu} p_{b \alpha} \Sigma_{a}^{\alpha \mu} \\
& \left.+2 p_{a} \cdot p_{b} p_{a}^{2} p_{b}^{\nu} p_{b \alpha} \Sigma_{a}^{\alpha \mu}+2\left(p_{a} \cdot p_{b}\right)^{2} p_{a}^{\mu} p_{b \alpha} \Sigma_{a}^{\alpha \nu}-2 p_{a} \cdot p_{b} p_{a}^{2} p_{b}^{\mu} p_{b \alpha} \Sigma_{a}^{\alpha \nu}\right\} \\
& -p_{a} \cdot k\left\{-2 p_{b}^{2} p_{a}^{\mu} p_{b}^{\nu} p_{a} \cdot r_{a} p_{a} \cdot p_{b}+p_{a}^{2} p_{b}^{2} p_{a}^{\mu} p_{b}^{\nu} p_{b} \cdot r_{a}+10 p_{b}^{2} p_{b}^{\mu} p_{a}^{\nu} p_{a} \cdot r_{a} p_{a} \cdot p_{b}\right. \\
& -7 p_{a}^{2} p_{b}^{2} p_{b}^{\mu} p_{a}^{\nu} p_{b} \cdot r_{a}+2 p_{a} \cdot p_{b} p_{b}^{\mu} p_{b}^{\nu} p_{b} \cdot r_{a} p_{a}^{2}-2\left(p_{a} \cdot p_{b}\right)^{2} p_{b}^{\mu} p_{b}^{\nu} p_{a} \cdot r_{a}+2\left(p_{a} \cdot p_{b}\right)^{2} p_{b}^{\nu} p_{a}^{\mu} p_{b} \cdot r_{a} \\
& -2\left(p_{a} \cdot p_{b}\right)^{3} p_{b}^{\nu} r_{a}^{\mu}+p_{a}^{2} p_{b}^{2} p_{a} \cdot p_{b} p_{b}^{\nu} r_{a}^{\mu}-3\left(p_{b}^{2}\right)^{2} p_{a} \cdot r_{a} p_{a}^{\mu} p_{a}^{\nu}+\left(p_{b}^{2}\right)^{2} p_{a}^{2} p_{a}^{\nu} r_{a}^{\mu} \\
& -2\left(p_{a} \cdot p_{b}\right)^{2} p_{b} \cdot r_{a} p_{b}^{\mu} p_{a}^{\nu}+2\left(p_{a} \cdot p_{b}\right)^{3} p_{b}^{\mu} r_{a}^{\nu}-3 p_{a}^{2} p_{b}^{2} p_{a} \cdot p_{b} p_{b}^{\mu} r_{a}^{\nu}+2 p_{a}^{2}\left(p_{b}^{2}\right)^{2} p_{a}^{\mu} r_{a}^{\nu} \\
& +p_{b}^{2} p_{a} \cdot p_{b} p_{a}^{\mu} p_{b} \cdot r_{a} p_{a}^{\nu}-p_{b}^{2}\left(p_{a} \cdot p_{b}\right)^{2} p_{a}^{\mu} r_{a}^{\nu}-p_{b}^{2}\left\{\left(p_{a} \cdot p_{b}\right)^{2}-p_{a}^{2} p_{b}^{2}\right\} J_{a}^{\mu \nu}+2\left(p_{a} \cdot p_{b}\right)^{2} p_{b}^{\nu} p_{b \alpha} \Sigma_{a}^{\alpha \mu} \\
& \left.\left.-2\left(p_{a} \cdot p_{b}\right)^{2} p_{b}^{\mu} p_{b \alpha} \Sigma_{a}^{\alpha \nu}+p_{b}^{2} p_{a} \cdot p_{b} p_{a}^{\mu} p_{b \alpha} \Sigma_{a}^{\alpha \nu}-p_{a}^{2} p_{b}^{2} p_{b}^{\mu} p_{b \alpha} \Sigma_{a}^{\alpha \nu}\right\}\right] \text {. }
\end{aligned}
$$

After summing over the contributions of Eqs. (B13), (B17), and (B15) we find the following order $\mathcal{O}(\omega \ln \omega)$ contribution from the gravitational energy-momentum tensor expression (3.53):

$$
\begin{aligned}
\Delta_{(1)}^{(\omega \ln \omega)} \hat{T}_{2}^{h \mu \nu}(k)= & \frac{i G}{2} \sum_{\substack{a b b=1 \\
b \neq a \\
\eta_{a} \eta_{b}=1}}^{M+N} \frac{1}{\left[\left(p_{a} \cdot p_{b}\right)^{2}-p_{a}^{2} p_{b}^{2}\right]^{5 / 2}} \ln \left\{L\left(\omega+i \epsilon \eta_{a}\right)\right\} \\
& \times\left[p _ { b } \cdot k \left\{6\left(p_{a} \cdot p_{b}\right)^{3} p_{b}^{\mu} p_{a}^{\nu} p_{b} \cdot r_{a} p_{a}^{2}-6\left(p_{a} \cdot p_{b}\right)^{4} p_{b}^{\mu} p_{a}^{\nu} p_{a} \cdot r_{a}-3\left(p_{a} \cdot p_{b}\right)^{2} p_{a}^{2} p_{b}^{2} p_{a}^{\mu} p_{a}^{\nu} p_{b} \cdot r_{a}\right.\right. \\
& +3\left(p_{a} \cdot p_{b}\right)^{3} p_{b}^{2} p_{a}^{\mu} p_{a}^{\nu} p_{a} \cdot r_{a}-6\left(p_{a} \cdot p_{b}\right)^{2}\left(p_{a}^{2}\right)^{2} p_{b}^{\mu} p_{b}^{\nu} p_{b} \cdot r_{a}+6\left(p_{a} \cdot p_{b}\right)^{3} p_{a}^{2} p_{b}^{\mu} p_{b}^{\nu} p_{a} \cdot r_{a} \\
& +3 p_{a} \cdot p_{b}\left(p_{a}^{2}\right)^{2} p_{b}^{2} p_{a}^{\mu} p_{b}^{\nu} p_{b} \cdot r_{a}-3\left(p_{a} \cdot p_{b}\right)^{2} p_{a}^{2} p_{b}^{2} p_{a}^{\mu} p_{b}^{\nu} p_{a} \cdot r_{a}-3 p_{a} \cdot p_{b}\left(p_{a}^{2}\right)^{2} p_{b}^{2} p_{b}^{\mu} p_{a}^{\nu} p_{b} \cdot r_{a} \\
& \left.+3\left(p_{a} \cdot p_{b}\right)^{2} p_{a}^{2} p_{b}^{2} p_{b}^{\mu} p_{a}^{\nu} p_{a} \cdot r_{a}+3 p_{b}^{2}\left(p_{a}^{2}\right)^{3} p_{b}^{\mu} p_{b}^{\nu} p_{b} \cdot r_{a}-3 p_{b}^{2}\left(p_{a}^{2}\right)^{2} p_{a} \cdot p_{b} p_{b}^{\mu} p_{b}^{\nu} p_{a} \cdot r_{a}\right\} \\
& -p_{a} \cdot k\left\{3 p_{b}^{2}\left(p_{a} \cdot p_{b}\right)^{3} p_{a}^{\mu} p_{b}^{\nu} p_{a} \cdot r_{a}-3 p_{b}^{2}\left(p_{a} \cdot p_{b}\right)^{2} p_{a}^{\mu} p_{b}^{\nu} p_{a}^{2} p_{b} \cdot r_{a}+3 p_{a}^{2} p_{b}^{2}\left(p_{a} \cdot p_{b}\right)^{2} p_{b}^{\mu} p_{b}^{\nu} p_{a} \cdot r_{a}\right. \\
& -3\left(p_{a}^{2}\right)^{2} p_{b}^{2} p_{a} \cdot p_{b} p_{b}^{\mu} p_{b}^{\nu} p_{b} \cdot r_{a}-3\left(p_{b}^{2}\right)^{2}\left(p_{a} \cdot p_{b}\right)^{2} p_{a}^{\mu} p_{a}^{\nu} p_{a} \cdot r_{a}+3\left(p_{b}^{2}\right)^{2} p_{a}^{2} p_{a} \cdot p_{b} p_{a}^{\mu} p_{a}^{\nu} p_{b} \cdot r_{a}
\end{aligned}
$$




$$
\begin{aligned}
& -3\left(p_{b}^{2}\right)^{2} p_{a}^{2} p_{a} \cdot p_{b} p_{b}^{\mu} p_{a}^{\nu} p_{a} \cdot r_{a}+3\left(p_{a}^{2}\right)^{2}\left(p_{b}^{2}\right)^{2} p_{b}^{\mu} p_{a}^{\nu} p_{b} \cdot r_{a}+6\left(p_{a} \cdot p_{b}\right)^{3} p_{b}^{\mu} p_{b}^{\nu} p_{a}^{2} p_{b} \cdot r_{a} \\
& \left.\left.-6\left(p_{a} \cdot p_{b}\right)^{4} p_{b}^{\mu} p_{b}^{\nu} p_{a} \cdot r_{a}-6\left(p_{a} \cdot p_{b}\right)^{2} p_{a}^{2} p_{b}^{2} p_{b}^{\mu} p_{a}^{\nu} p_{b} \cdot r_{a}+6\left(p_{a} \cdot p_{b}\right)^{3} p_{b}^{2} p_{b}^{\mu} p_{a}^{\nu} p_{a} \cdot r_{a}\right\}\right] \\
& +\frac{i G}{2} \sum_{\substack{a, b=1 \\
b \neq a \\
\eta_{a} \eta_{b}=1}}^{M+N} \frac{1}{\left[\left(p_{a} \cdot p_{b}\right)^{2}-p_{a}^{2} p_{b}^{2}\right]^{3 / 2}} \ln \left\{L\left(\omega+i \epsilon \eta_{a}\right)\right\} \\
& \times\left[-2 p_{a} \cdot p_{b} p_{b} \cdot k p_{b} \cdot r_{a} p_{a}^{2} p_{a}^{\mu} p_{b}^{\nu}+5 p_{b}^{2} p_{a} \cdot k p_{a} \cdot p_{b} p_{a} \cdot r_{a} p_{a}^{\mu} p_{b}^{\nu}-4\left(p_{a} \cdot p_{b}\right)^{2} p_{b} \cdot r_{a} p_{a} \cdot k p_{a}^{\mu} p_{b}^{\nu}\right. \\
& +2\left(p_{a} \cdot p_{b}\right)^{3} r_{a} \cdot k p_{a}^{\mu} p_{b}^{\nu}-3 p_{a}^{2} p_{b}^{2} p_{a} \cdot p_{b} r_{a} \cdot k p_{a}^{\mu} p_{b}^{\nu} \\
& -p_{a}^{2} p_{b}^{2} p_{b} \cdot k p_{a} \cdot r_{a} p_{a}^{\mu} p_{b}^{\nu}-8 p_{a} \cdot p_{b} p_{b} \cdot k p_{a}^{2} p_{b} \cdot r_{a} p_{a}^{\nu} p_{b}^{\mu}+10\left(p_{a} \cdot p_{b}\right)^{2} p_{b} \cdot k p_{a} \cdot r_{a} p_{a}^{\nu} p_{b}^{\mu} \\
& -2 p_{a}^{2} p_{b}^{2} p_{b} \cdot r_{a} p_{a} \cdot k p_{a}^{\nu} p_{b}^{\mu}-4\left(p_{a} \cdot p_{b}\right)^{2} p_{b} \cdot r_{a} p_{a} \cdot k p_{a}^{\nu} p_{b}^{\mu}-3 p_{a}^{2} p_{b}^{2} p_{a} \cdot p_{b} r_{a} \cdot k p_{a}^{\nu} p_{b}^{\mu} \\
& +8 p_{a} \cdot p_{b} p_{b}^{2} p_{a} \cdot r_{a} p_{a} \cdot k p_{a}^{\nu} p_{b}^{\mu}+2\left(p_{a} \cdot p_{b}\right)^{3} r_{a} \cdot k p_{a}^{\nu} p_{b}^{\mu}-10\left(p_{a} \cdot p_{b}\right)^{2} p_{a} \cdot r_{a} p_{a} \cdot k p_{b}^{\mu} p_{b}^{\nu} \\
& +8 p_{a} \cdot p_{b} p_{a}^{2} p_{b} \cdot r_{a} p_{a} \cdot k p_{b}^{\mu} p_{b}^{\nu}-4 p_{b} \cdot k p_{a}^{2} p_{a} \cdot r_{a} p_{a} \cdot p_{b} p_{b}^{\mu} p_{b}^{\nu}+4 p_{b} \cdot k\left(p_{a}^{2}\right)^{2} p_{b} \cdot r_{a} p_{b}^{\mu} p_{b}^{\nu} \\
& +2\left(p_{a} \cdot p_{b}\right)^{3} p_{a} \cdot k p_{b}^{\mu} r_{a}^{\nu}-3 p_{a}^{2} p_{b}^{2} p_{a} \cdot p_{b} p_{a} \cdot k p_{b}^{\mu} r_{a}^{\nu}+2 p_{a} \cdot k\left(p_{a} \cdot p_{b}\right)^{3} p_{b}^{\nu} r_{a}^{\mu} \\
& -3 p_{a} \cdot k p_{a}^{2} p_{b}^{2} p_{a} \cdot p_{b} p_{b}^{\nu} r_{a}^{\mu}-2 p_{b} \cdot k\left(p_{a} \cdot p_{b}\right)^{2} p_{a}^{2} p_{b}^{\nu} r_{a}^{\mu}+\left(p_{a}^{2}\right)^{2} p_{b}^{2} p_{b}^{\nu} r_{a}^{\mu} p_{b} \cdot k \\
& +4 p_{b} \cdot k\left(p_{a} \cdot p_{b}\right)^{2} p_{b} \cdot r_{a} p_{a}^{\mu} p_{a}^{\nu}-5 p_{b} \cdot k p_{a} \cdot p_{b} p_{b}^{2} p_{a} \cdot r_{a} p_{a}^{\mu} p_{a}^{\nu}-2 p_{b}^{2} p_{a} \cdot p_{b} p_{a} \cdot k p_{b} \cdot r_{a} p_{a}^{\mu} p_{a}^{\nu} \\
& -\left(p_{b}^{2}\right)^{2} p_{a} \cdot k p_{a} \cdot r_{a} p_{a}^{\mu} p_{a}^{\nu}-2 p_{b} \cdot k\left(p_{a} \cdot p_{b}\right)^{3} p_{a}^{\nu} r_{a}^{\mu}+3 p_{b} \cdot k p_{a}^{2} p_{b}^{2} p_{a} \cdot p_{b} p_{a}^{\nu} r_{a}^{\mu} \\
& +2 p_{b}^{2}\left(p_{a} \cdot p_{b}\right)^{2} p_{a} \cdot k p_{a}^{\nu} r_{a}^{\mu}-p_{a}^{2}\left(p_{b}^{2}\right)^{2} p_{a} \cdot k p_{a}^{\nu} r_{a}^{\mu}-2 p_{b} \cdot k\left(p_{a} \cdot p_{b}\right)^{3} p_{a}^{\mu} r_{a}^{\nu} \\
& +3 p_{b} \cdot k p_{a}^{2} p_{b}^{2} p_{a} \cdot p_{b} p_{a}^{\mu} r_{a}^{\nu}+2 p_{b}^{2}\left(p_{a} \cdot p_{b}\right)^{2} p_{a} \cdot k p_{a}^{\mu} r_{a}^{\nu}-p_{a}^{2}\left(p_{b}^{2}\right)^{2} p_{a} \cdot k p_{a}^{\mu} r_{a}^{\nu} \\
& +2\left(p_{a} \cdot p_{b}\right)^{2} p_{a}^{2} r_{a} \cdot k p_{b}^{\mu} p_{b}^{\nu}-2\left(p_{a} \cdot p_{b}\right)^{2} p_{b}^{2} r_{a} \cdot k p_{a}^{\mu} p_{a}^{\nu}+p_{a}^{2} p_{b}^{2} p_{a} \cdot r_{a} p_{a} \cdot k p_{b}^{\mu} p_{b}^{\nu} \\
& -\left(p_{a}^{2}\right)^{2} p_{b}^{2} r_{a} \cdot k p_{b}^{\mu} p_{b}^{\nu}+3 p_{a}^{2}\left(p_{b}^{2}\right)^{2} r_{a} \cdot k p_{a}^{\mu} p_{a}^{\nu}-p_{b} \cdot k p_{a}^{2} p_{b}^{2} p_{b} \cdot r_{a} p_{a}^{\mu} p_{a}^{\nu} \\
& -2 p_{b} \cdot k\left(p_{a} \cdot p_{b}\right)^{2} p_{a}^{2} p_{b}^{\mu} r_{a}^{\nu}-p_{b} \cdot k p_{a}^{2} p_{b}^{2} p_{a} \cdot r_{a} p_{b}^{\mu} p_{a}^{\nu}+p_{b} \cdot k\left(p_{a}^{2}\right)^{2} p_{b}^{2} p_{b}^{\mu} r_{a}^{\nu} \\
& +4 p_{b} \cdot k\left(p_{a} \cdot p_{b}\right)^{2} p_{a} \cdot r_{a} p_{a}^{\mu} p_{b}^{\nu}+p_{a} \cdot k p_{a}^{2} p_{b}^{2} p_{b} \cdot r_{a} p_{a}^{\mu} p_{b}^{\nu} \\
& -2\left\{\left(p_{a} \cdot p_{b}\right)^{2}-p_{a}^{2} p_{b}^{2}\right\} p_{a}^{\mu} p_{b}^{\nu} p_{b \alpha} \Sigma_{a}^{\alpha \beta} k_{\beta}-2\left\{\left(p_{a} \cdot p_{b}\right)^{2}-p_{a}^{2} p_{b}^{2}\right\} p_{a}^{\nu} p_{b}^{\mu} p_{b \alpha} \Sigma_{a}^{\alpha \beta} k_{\beta} \\
& -2\left\{\left(p_{a} \cdot p_{b}\right)^{2}-p_{a}^{2} p_{b}^{2}\right\} p_{a} \cdot k p_{b}^{\mu} p_{b \alpha} \Sigma_{a}^{\alpha \nu}-2\left\{\left(p_{a} \cdot p_{b}\right)^{2}-p_{a}^{2} p_{b}^{2}\right\} p_{a} \cdot k p_{b}^{\nu} p_{b \alpha} \Sigma_{a}^{\alpha \mu} \\
& +2\left\{\left(p_{a} \cdot p_{b}\right)^{2}-p_{a}^{2} p_{b}^{2}\right\} p_{b} \cdot k p_{a}^{\nu} p_{b \alpha} \Sigma_{a}^{\alpha \mu}+2\left\{\left(p_{a} \cdot p_{b}\right)^{2}-p_{a}^{2} p_{b}^{2}\right\} p_{b} \cdot k p_{a}^{\mu} p_{b \alpha} \Sigma_{a}^{\alpha \nu} \\
& +2\left\{\left(p_{a} \cdot p_{b}\right)^{2}-p_{a}^{2} p_{b}^{2}\right\} p_{b}^{2} p_{a}^{\nu} \Sigma_{a}^{\mu \alpha} k_{\alpha}+2\left\{\left(p_{a} \cdot p_{b}\right)^{2}-p_{a}^{2} p_{b}^{2}\right\} p_{b}^{2} p_{a}^{\mu} \Sigma_{a}^{\nu \alpha} k_{\alpha} \\
& +2 p_{a} \cdot p_{b} p_{a}^{2} p_{b}^{\mu} p_{b}^{\nu} p_{b \alpha} \Sigma_{a}^{\alpha \beta} k_{\beta}-2 p_{a} \cdot p_{b} p_{a}^{2} p_{b} \cdot k p_{b}^{\mu} p_{b \alpha} \Sigma_{a}^{\alpha \nu}-2 p_{b}^{2} p_{a} \cdot p_{b} p_{a}^{\mu} p_{a}^{\nu} p_{b \alpha} \Sigma_{a}^{\alpha \beta} k_{\beta} \\
& \left.-2 p_{b} \cdot k p_{a} \cdot p_{b} p_{a}^{2} p_{b}^{\nu} p_{b \alpha} \Sigma_{a}^{\alpha \mu}\right] \text {. }
\end{aligned}
$$

\section{APPENDIX C: SOFT RADIATION FROM HIGH FREQUENCY GRAVITATIONAL WAVE EMISSION}

Let us first write down the expression of Eq. (3.45) with the replacement of $G_{r}(k-\ell) G_{r}(\ell)$ by $-2 \pi i \delta\left(\ell^{2}\right)\left[H\left(\ell^{0}\right)-H\left(-\ell^{0}\right)\right] G_{r}(k-\ell)$,

$$
\begin{aligned}
\hat{T}_{\text {extra }}^{h \mu \nu}(k) \equiv & (8 \pi G)(2 \pi i) \sum_{a, b=1}^{M+N} \int \frac{d^{4} \ell}{(2 \pi)^{4}} \delta\left(\ell^{2}\right)\left[H\left(\ell^{0}\right)-H\left(-\ell^{0}\right)\right] G_{r}(k-\ell) \frac{1}{p_{b} \cdot \ell-i \epsilon} \frac{1}{p_{a} \cdot(k-\ell)-i \epsilon} \\
& \times\left\{p_{b \alpha} p_{b \beta}-\frac{1}{2} p_{b}^{2} \eta_{\alpha \beta}+i p_{b(\alpha} J_{b, \beta) \gamma} \ell^{\gamma}-\frac{i}{2} \eta_{\alpha \beta} p_{b}^{\delta} J_{b, \delta \gamma} \ell^{\gamma}\right\} \mathcal{F}^{\mu \nu, \alpha \beta, \rho \sigma}(k, \ell) \\
& \times\left\{p_{a \rho} p_{a \sigma}-\frac{1}{2} p_{a}^{2} \eta_{\rho \sigma}+i p_{a(\rho} J_{a, \sigma) \delta}(k-\ell)^{\delta}-\frac{i}{2} \eta_{\rho \sigma} p_{a}^{\kappa} J_{a, \kappa \tau}(k-\ell)^{\tau}\right\} .
\end{aligned}
$$

We want to analyze the above expression in the integration region $\omega \ll\left|\ell^{\mu}\right| \ll L^{-1}$. Because of the presence of $\left[H\left(\ell^{0}\right)-\right.$ $\left.H\left(-\ell^{0}\right)\right]$ inside the integrand, the part of the integrand containing even number of $\ell$ vanishes. On the other hand, to produce 
a $\ln \omega$ factor we need an integrand with four power of $\ell$ in the denominator, which will vanish due to the presence of $\left[H\left(\ell^{0}\right)-H\left(-\ell^{0}\right)\right]$. Hence just from this argument it is clear that we cannot receive any nonvanishing $\ln \omega$ or $\omega \ln \omega$ contribution from the above expression. Still for completeness, let us analyze the nonvanishing contribution of the above expression up to order $\mathcal{O}\left(\omega^{0}\right)$ in the integration range $\omega \ll\left|\ell^{\mu}\right| \ll L^{-1}$ :

$$
\begin{aligned}
\Delta \hat{T}_{\mathrm{extra}}^{h \mu \nu}(k)= & -(8 \pi G)(2 \pi i) \sum_{a, b=1}^{M+N} \int_{\omega}^{L^{-1}} \frac{d^{4} \ell}{(2 \pi)^{4}} \delta\left(\ell^{2}\right)\left[H\left(\ell^{0}\right)-H\left(-\ell^{0}\right)\right] \frac{1}{2 k \cdot \ell-i \epsilon} \frac{1}{p_{b} \cdot \ell-i \epsilon} \frac{1}{p_{a} \cdot \ell+i \epsilon} \\
& \times\left[\left\{p_{b \alpha} p_{b \beta}-\frac{1}{2} p_{b}^{2} \eta_{\alpha \beta}\right\} \Delta_{(\ell \ell)} \mathcal{F}^{\mu \nu, \alpha \beta, \rho \sigma}(k, \ell)\left\{p_{a \rho} p_{a \sigma}-\frac{1}{2} p_{a}^{2} \eta_{\rho \sigma}+i p_{a(\rho} J_{a, \sigma) \delta} k^{\delta}-\frac{i}{2} \eta_{\rho \sigma} p_{a}^{\kappa} J_{a, \kappa \tau} k^{\tau}\right\}\right. \\
& -\left\{p_{b \alpha} p_{b \beta}-\frac{1}{2} p_{b}^{2} \eta_{\alpha \beta}\right\} \Delta_{(k \ell)} \mathcal{F}^{\mu \nu, \alpha \beta, \rho \sigma}(k, \ell)\left\{i p_{a(\rho} J_{a, \sigma) \delta} \ell^{\delta}-\frac{i}{2} \eta_{\rho \sigma} p_{a}^{\kappa} J_{a, \kappa \tau} \ell^{\tau}\right\} \\
& \left.+\left\{i p_{b(\alpha} J_{b, \beta) \gamma} \ell^{\gamma}-\frac{i}{2} \eta_{\alpha \beta} p_{b}^{\delta} J_{b, \delta \gamma} \ell^{\gamma}\right\} \Delta_{(k \ell)} \mathcal{F}^{\mu \nu, \alpha \beta, \rho \sigma}(k, \ell)\left\{p_{a \rho} p_{a \sigma}-\frac{1}{2} p_{a}^{2} \eta_{\rho \sigma}\right\}\right] \\
& -(8 \pi G)(2 \pi i) \sum_{a, b=1}^{M+N} \int_{\omega}^{L^{-1}} \frac{d^{4} \ell}{(2 \pi)^{4}} \delta\left(\ell^{2}\right)\left[H\left(\ell^{0}\right)-H\left(-\ell^{0}\right)\right] \frac{1}{2 k \cdot \ell-i \epsilon} \frac{1}{p_{b} \cdot \ell-i \epsilon} \frac{p_{a} \cdot k}{\left(p_{a} \cdot \ell+i \epsilon\right)^{2}} \\
& \times\left[-\left\{p_{b \alpha} p_{b \beta}-\frac{1}{2} p_{b}^{2} \eta_{\alpha \beta}\right\} \Delta_{(\ell \ell)} \mathcal{F}^{\mu \nu, \alpha \beta, \rho \sigma}(k, \ell)\left\{i p_{a(\rho} J_{a, \sigma) \delta} \ell^{\delta}-\frac{i}{2} \eta_{\rho \sigma} p_{a}^{\kappa} J_{a, \kappa \tau} \ell^{\tau}\right\}\right. \\
& \left.+\left\{i p_{b(\alpha} J_{b, \beta) \gamma} \ell^{\gamma}-\frac{i}{2} \eta_{\alpha \beta} p_{b}^{\delta} J_{b, \delta \gamma} \ell^{\gamma}\right\} \Delta_{(\ell \ell)} \mathcal{F}^{\mu \nu, \alpha \beta, \rho \sigma}(k, \ell)\left\{p_{a \rho} p_{a \sigma}-\frac{1}{2} p_{a}^{2} \eta_{\rho \sigma}\right\}\right] .
\end{aligned}
$$

Now in the above expression after the contraction of various terms within the square bracket, we find some terms containing $\ell^{2}$, which will vanish due to the presence of $\delta\left(\ell^{2}\right)$. On the other hand, if we get $p_{b} . \ell$ (or $p_{a} \cdot \ell$ ), then it cancels with the denominator $\left\{p_{b} . \ell-i \epsilon\right\}^{-1}$ (or $\left\{p_{a} \cdot \ell+i \epsilon\right\}^{-1}$, and if only one such denominator is present, then after cancellation of it the rest of the coefficient vanishes after using $\sum_{b} p_{b}^{\alpha}=0$ or $\sum_{b} J_{b}^{\alpha \beta}=0$ (or $\sum_{a} p_{a}^{\alpha}=0$ or $\sum_{a} J_{a}^{\alpha \beta}=0$ ). Hence after eliminating those terms and interchanging $a \leftrightarrow b$ in some places, ${ }^{14}$ the above expression simplifies to

$$
\begin{aligned}
\Delta \hat{T}_{\mathrm{extra}}^{h \mu \nu}(k)= & -(8 \pi G)(2 \pi i) \sum_{a, b=1}^{M+N} \int_{\omega}^{L^{-1}} \frac{d^{4} \ell}{(2 \pi)^{4}} \delta\left(\ell^{2}\right) H\left(\ell^{0}\right) \frac{\ell^{\mu} \ell^{\nu}}{k \cdot \ell-i \epsilon} \frac{1}{p_{b} \cdot \ell-i \epsilon} \frac{1}{p_{a} \cdot \ell+i \epsilon}\left\{\left(p_{a} \cdot p_{b}\right)^{2}-\frac{1}{2} p_{a}^{2} p_{b}^{2}\right\} \\
& +\left(8 \pi^{2} G\right) \sum_{a, b=1}^{M+N} \int_{\omega}^{L^{-1}} \frac{d^{4} \ell}{(2 \pi)^{4}} \delta\left(\ell^{2}\right) H\left(\ell^{0}\right) \frac{1}{k \cdot \ell-i \epsilon} \frac{1}{p_{b} \cdot \ell-i \epsilon} \frac{1}{p_{a} \cdot \ell+i \epsilon}\left\{2 \ell^{\mu} \ell^{\nu} p_{a} \cdot p_{b} p_{a \rho} J_{b}^{\rho \sigma} k_{\sigma}\right. \\
& -p_{a}^{2} \ell^{\mu} \ell^{\nu} p_{b \rho} J_{b}^{\rho \sigma} k_{\sigma}+2 p_{a} \cdot p_{b} p_{a}^{\mu} \ell^{\nu} k_{\alpha} J_{b}^{\alpha \beta} \ell_{\beta}+2 p_{b} \cdot k p_{a}^{\mu} \ell^{\nu} p_{a \alpha} J_{b}^{\alpha \beta} \ell_{\beta}+2 p_{a} \cdot p_{b} p_{a}^{\nu} \ell^{\mu} k_{\alpha} J_{b}^{\alpha \beta} \ell_{\beta} \\
& +2 p_{b} \cdot k p_{a}^{\nu} \ell^{\mu} p_{a \alpha} J_{b}^{\alpha \beta} \ell_{\beta}-2 p_{a} \cdot k p_{a} \cdot p_{b} \ell^{\nu} J_{b}^{\mu \alpha} \ell_{\alpha}-2 p_{a} \cdot k p_{b}^{\mu} \ell^{\nu} p_{a \alpha} J_{b}^{\alpha \beta} \ell_{\beta}-2 p_{a} \cdot k p_{a} \cdot p_{b} \ell^{\mu} J_{b}^{\nu \alpha} \ell_{\alpha} \\
& \left.-2 p_{b}^{\nu} \ell^{\mu} p_{a} \cdot k p_{a \alpha} J_{b}^{\alpha \beta} \ell_{\beta}\right\} \\
& +\left(8 \pi^{2} G\right) \sum_{a, b=1}^{M+N} \int_{\omega}^{L^{-1}} \frac{d^{4} \ell}{(2 \pi)^{4}} \delta\left(\ell^{2}\right) H\left(\ell^{0}\right) \frac{1}{k \cdot \ell-i \epsilon} \frac{1}{p_{b} \cdot \ell-i \epsilon} \frac{p_{a} \cdot k}{\left(p_{a} \cdot \ell+i \epsilon\right)^{2}} \\
& \times\left[2 \ell^{\mu} \ell^{\nu} p_{a} \cdot p_{b} p_{a \alpha} J_{b}^{\alpha \beta} \ell_{\beta}-p_{a}^{2} \ell^{\mu} \ell^{\nu} p_{b \alpha} J_{b}^{\alpha \beta} \ell_{\beta}-2 \ell^{\mu} \ell^{\nu} p_{a} \cdot p_{b} p_{b \rho} J_{a}^{\rho \sigma} \ell_{\sigma}+p_{b}^{2} \ell^{\mu} \ell^{\nu} p_{a \rho} J_{a}^{\rho \sigma} \ell_{\sigma}\right] \\
& +\left(8 \pi^{2} G\right) \sum_{a, b=1}^{M+N} \int_{\omega}^{L^{-1}} \frac{d^{4} \ell}{(2 \pi)^{4}} \delta\left(\ell^{2}\right) H\left(\ell^{0}\right) \frac{1}{p_{b} \cdot \ell-i \epsilon} \frac{1}{p_{a} \cdot \ell+i \epsilon}\left\{4 p_{a}^{\mu} p_{a}^{\nu} p_{b \alpha} J_{b}^{\alpha \beta} \ell_{\beta}+2 p_{a} \cdot p_{b} p_{a}^{\mu} J_{b}^{\nu \alpha} \ell_{\alpha}\right. \\
& \left.+2 p_{b}^{\nu} p_{a}^{\mu} p_{a \alpha} J_{b}^{\alpha \beta} \ell_{\beta}-2 p_{a}^{2} p_{b}^{\mu} J_{b}^{\nu \alpha} \ell_{\alpha}-2 p_{a}^{2} p_{b}^{\nu} J_{b}^{\mu \alpha} \ell_{\alpha}-2 p_{b}^{\mu} p_{a}^{\nu} p_{a \alpha} J_{b}^{\alpha \beta} \ell_{\beta}-2 p_{a} \cdot p_{b} p_{a}^{\nu} J_{b}^{\mu \alpha} \ell_{\alpha}\right\} .
\end{aligned}
$$

\footnotetext{
${ }^{14}$ Though the signs of $i \epsilon$ are different for denominators between $p_{a} . \ell$ and $p_{b} . \ell$, still the $a \leftrightarrow b$ interchange makes sense as the integrals have to evaluate with $\delta\left(\ell^{2}\right)$. Hence we can set $\epsilon=0$ from the beginning.
} 
The first line in the above expression has been identified with leading order soft radiation from real hard gravitational radiation in Appendix B of [13]. So here generalizing Appendix B of [13] to the next order, we try to understand whether the rest of the terms above can be understood as subleading order soft radiation from real hard gravitational radiation. Taking care of gravitational flux up to subleading order, the energy-momentum tensor for soft gravitational radiation with momentum $k$ becomes

$$
\begin{aligned}
\hat{T}_{R}^{\mu \nu}(k)= & \frac{G}{\pi^{2}} \sum_{a, b=1}^{M+N} \int d^{4} \ell \delta\left(\ell^{2}\right) H\left(\ell^{0}\right)\left[\frac{p_{a}^{\rho} p_{a}^{\sigma}-i p_{a}^{(\rho}(k-\ell)_{\alpha} J_{a}^{\alpha \sigma)}}{p_{a} \cdot(\ell-k)+i \epsilon}\right]\left[\frac{p_{b}^{\kappa} p_{b}^{\tau}-i p_{b}^{(\kappa} \ell_{\beta} J_{b}^{\beta \tau)}}{p_{b} \cdot \ell-i \epsilon}\right] \\
& \times \frac{\ell^{\mu} \ell^{\nu} \sum_{r} \varepsilon_{\rho \sigma}^{r} \varepsilon_{\kappa \tau}^{r *}-i \ell^{(\mu} k_{\gamma}\left[\Sigma_{g}^{\gamma \nu)}\right]_{\rho \sigma, \kappa \tau}}{i(\ell \cdot k-i \epsilon)}
\end{aligned}
$$

where the polarization sum and spin tensor of the soft gravitational field are given by

$$
\begin{gathered}
\sum_{r} \varepsilon_{\rho \sigma}^{r} \varepsilon_{\kappa \tau}^{r *}=\frac{1}{2}\left(\eta_{\rho \kappa} \eta_{\sigma \tau}+\eta_{\rho \tau} \eta_{\sigma \kappa}-\eta_{\rho \sigma} \eta_{\kappa \tau}\right), \\
{\left[\Sigma_{g}^{\gamma \nu)}\right]_{\rho \sigma, \kappa \tau}=-\frac{i}{2}\left[\eta_{\rho \kappa}\left(\delta_{\sigma}^{\gamma} \delta_{\tau}^{\nu}-\delta_{\sigma}^{\nu} \delta_{\tau}^{\gamma}\right)+\eta_{\rho \tau}\left(\delta_{\sigma}^{\gamma} \delta_{\kappa}^{\nu}-\delta_{\sigma}^{\nu} \delta_{\kappa}^{\gamma}\right)+\eta_{\sigma \kappa}\left(\delta_{\rho}^{\gamma} \delta_{\tau}^{\nu}-\delta_{\rho}^{\nu} \delta_{\tau}^{\gamma}\right)+\eta_{\sigma \tau}\left(\delta_{\rho}^{\gamma} \delta_{\kappa}^{\nu}-\delta_{\rho}^{\nu} \delta_{\kappa}^{\gamma}\right)\right] .}
\end{gathered}
$$

Substituting the above results in Eq. (C4) and analyzing in the integration region $\omega \ll\left|\ell^{\mu}\right| \ll L^{-1}$, we get the following nonvanishing contribution up to order $\omega^{0}$ :

$$
\begin{aligned}
\hat{T}_{R}^{\mu \nu}(k)= & -\frac{i G}{\pi^{2}} \sum_{a, b=1}^{M+N} \int_{\omega}^{L^{-1}} d^{4} \ell \delta\left(\ell^{2}\right) H\left(\ell^{0}\right) \frac{\ell^{\mu} \ell^{\nu}}{k \cdot \ell-i \epsilon} \frac{1}{p_{b} \cdot \ell-i \epsilon} \frac{1}{p_{a} \cdot \ell+i \epsilon}\left\{\left(p_{a} \cdot p_{b}\right)^{2}-\frac{1}{2} p_{a}^{2} p_{b}^{2}\right\} \\
& +\frac{G}{2 \pi^{2}} \sum_{a, b=1}^{M+N} \int_{\omega}^{L^{-1}} d^{4} \ell \delta\left(\ell^{2}\right) H\left(\ell^{0}\right) \frac{1}{k \cdot \ell-i \epsilon} \frac{1}{p_{b} \cdot \ell-i \epsilon} \frac{1}{p_{a} \cdot \ell+i \epsilon}\left\{2 \ell^{\mu} \ell^{\nu} p_{a} \cdot p_{b} p_{a \rho} J_{b}^{\rho \sigma} k_{\sigma}\right. \\
& -p_{a}^{2} \ell^{\mu} \ell^{\nu} p_{b \rho} J_{b}^{\rho \sigma} k_{\sigma}+2 p_{a} \cdot p_{b} p_{a}^{\mu} \ell^{\nu} k_{\alpha} J_{b}^{\alpha \beta} \ell_{\beta}+2 p_{b} \cdot k p_{a}^{\mu} \ell^{\nu} p_{a \alpha} J_{b}^{\alpha \beta} \ell_{\beta}+2 p_{a} \cdot p_{b} p_{a}^{\nu} \ell^{\mu} k_{\alpha} J_{b}^{\alpha \beta} \ell_{\beta} \\
& +2 p_{b} \cdot k p_{a}^{\nu} \ell^{\mu} p_{a \alpha} J_{b}^{\alpha \beta} \ell_{\beta}-2 p_{a} \cdot k p_{a} \cdot p_{b} \ell^{\nu} J_{b}^{\mu \alpha} \ell_{\alpha}-2 p_{a} \cdot k p_{b}^{\mu} \ell^{\nu} p_{a \alpha} J_{b}^{\alpha \beta} \ell_{\beta}-2 p_{a} \cdot k p_{a} \cdot p_{b} \ell^{\mu} J_{b}^{\nu \alpha} \ell_{\alpha} \\
& \left.-2 p_{b}^{\nu} \ell^{\mu} p_{a} \cdot k p_{a \alpha} J_{b}^{\alpha \beta} \ell_{\beta}\right\} \\
& +\frac{G}{2 \pi^{2}} \sum_{a, b=1}^{M+N} \int_{\omega}^{L^{-1}} d^{4} \ell \delta\left(\ell^{2}\right) H\left(\ell^{0}\right) \frac{\ell^{\mu} \ell^{\nu}}{k \cdot \ell-i \epsilon} \frac{1}{p_{b} \cdot \ell-i \epsilon} \frac{p_{a} \cdot k}{\left(p_{a} \cdot \ell+i \epsilon\right)^{2}} \\
& \times\left[\left\{2 p_{a} \cdot p_{b} p_{a \alpha} J_{b}^{\alpha \beta} \ell_{\beta}-p_{a}^{2} p_{b \alpha} J_{b}^{\alpha \beta} \ell_{\beta}\right\}-\left\{2 p_{a} \cdot p_{b} p_{b \rho} J_{a}^{\rho \sigma} \ell_{\sigma}-p_{b}^{2} p_{a \rho} J_{a}^{\rho \sigma} \ell_{\sigma}\right\}\right] .
\end{aligned}
$$

Now if we compare the above expression with Eq. (C3), we observe that leaving the last two lines of Eq. (C3), the rest of the terms match. But we do not need to worry about these extra terms as they do not contribute at order $\omega \ln \omega$.

[1] Y. B. Zel'dovich and A. G. Polnarev, Radiation of gravitational waves by a cluster of superdense stars, Astron. Zh. 51, 30 (1974) [Sov. Astron. 18, 17 (1974)].

[2] V. B. Braginsky and L. P. Grishchuk, Kinematic resonance and memory effect in free mass gravitational antennas, $\mathrm{Zh}$. Eksp. Teor. Fiz. 89, 744 (1985) [Sov. Phys. JETP 62, 427 (1985)].
[3] V. B. Braginskii and K.S. Thorne, Gravitational-wave bursts with memory and experimental prospects, Nature (London) 327, 123 (1987).

[4] P. N. Payne, Smarr's zero-frequency-limit calculation, Phys. Rev. D 28, 1894 (1983).

[5] M. Ludvigsen, Geodesic deviation at null infinity and the physical effects of very long wave gravitational radiation, Gen. Relativ. Gravit. 21, 1205 (1989). 
[6] D. Christodoulou, Nonlinear Nature of Gravitation and Gravitational Wave Experiments, Phys. Rev. Lett. 67, 1486 (1991).

[7] K. S. Thorne, Gravitational-wave bursts with memory: The Christodoulou effect, Phys. Rev. D 45, 520 (1992).

[8] L. Blanchet and T. Damour, Hereditary effects in gravitational radiation, Phys. Rev. D 46, 4304 (1992).

[9] J. B. Wang et al., Searching for gravitational wave memory bursts with the Parkes Pulsar Timing Array, Mon. Not. R. Astron. Soc. 446, 1657 (2015).

[10] M. Favata, The gravitational-wave memory effect, Classical Quant. Grav. 27, 084036 (2010).

[11] A. Laddha and A. Sen, Observational signature of the logarithmic terms in the soft graviton theorem, Phys. Rev. D 100, 024009 (2019).

[12] B. Sahoo and A. Sen, Classical and quantum results on logarithmic terms in the soft theorem in four dimensions, J. High Energy Phys. 02 (2019) 086.

[13] A. P. Saha, B. Sahoo, and A. Sen, Proof of the classical soft graviton theorem in $D=4$, J. High Energy Phys. 06 (2020) 153.

[14] B. Sahoo, Classical sub-subleading soft photon and soft graviton theorems in four spacetime dimensions, J. High Energy Phys. 12 (2020) 070.

[15] B. Sahoo and A. Sen, Classical soft graviton theorem rewritten, arXiv:2105.08739.

[16] A. Laddha and A. Sen, Gravity waves from soft Theorem in general dimensions, J. High Energy Phys. 09 (2018) 105.

[17] A. Laddha and A. Sen, Logarithmic terms in the soft expansion in four dimensions, J. High Energy Phys. 10 (2018) 056.

[18] S. Pasterski, A. Strominger, and A. Zhiboedov, New gravitational memories, J. High Energy Phys. 12 (2016) 053.

[19] A. Laddha and A. Sen, Sub-subleading soft graviton theorem in generic theories of quantum gravity, J. High Energy Phys. 10 (2017) 065.

[20] W. D. Goldberger and A. K. Ridgway, Radiation and the classical double copy for color charges, Phys. Rev. D 95, 125010 (2017).

[21] A. Laddha and A. Sen, Classical proof of the classical soft graviton theorem in D>4, Phys. Rev. D 101, 084011 (2020).

[22] W. D. Goldberger, J. Li, and S. G. Prabhu, Spinning particles, axion radiation, and the classical double copy, Phys. Rev. D 97, 105018 (2018).

[23] J. Li and S. G. Prabhu, Gravitational radiation from the classical spinning double copy, Phys. Rev. D 97, 105019 (2018).

[24] J. Vines, Scattering of two spinning black holes in postMinkowskian gravity, to all orders in spin, and effectiveone-body mappings, Classical Quant. Grav. 35, 084002 (2018).

[25] A. Guevara, A. Ochirov, and J. Vines, Scattering of spinning black holes from exponentiated soft factors, J. High Energy Phys. 09 (2019) 056.

[26] W. Tulczyjew, Motion of multipole particles in general relativity theory binaries, Acta Phys. Pol. 18, 393 (1959).
[27] W. G. Dixon, Extended bodies in general relativity: Their description and motion, in Proceedings of the International School of Physics Enrico Fermi LXVII, edited by J. Ehlers (North Holland, Amsterdam, 1979).

[28] R. A. Porto, Post-Newtonian corrections to the motion of spinning bodies in NRGR, Phys. Rev. D 73, 104031 (2006).

[29] W. D. Goldberger and I. Z. Rothstein, Dissipative effects in the worldline approach to black hole dynamics, Phys. Rev. D 73, 104030 (2006).

[30] R. A. Porto and I. Z. Rothstein, The Hyperfine EinsteinInfeld-Hoffmann Potential, Phys. Rev. Lett. 97, 021101 (2006).

[31] R. A. Porto and I. Z. Rothstein, Next to leading order spin(1) spin(1) effects in the motion of inspiralling compact binaries, Phys. Rev. D 78, 044013 (2008); Erratum, Phys. Rev. D 81, 029905 (2010).

[32] J. W. van Holten, Spinning bodies in general relativity, Int. J. Geom. Methods Mod. Phys. 13, 1640002 (2016).

[33] A. Papapetrou, Spinning test-particles in general relativity. I, Proc. R. Soc. A 209, 248 (1951).

[34] M. Mathisson, Das Zitternde Elektron und seine Dynamik, Acta Phys. Pol. 6, 167 (1937).

[35] R. Wald, Gravitational spin interaction, Phys. Rev. D 6, 406 (1972).

[36] G. Compère and A. Druart, Complete set of quasi-conserved quantities for spinning particles around Kerr, arXiv:2105 .12454

[37] A. D. Fokker, Relativiteitstheorie (P. Noordhoff, Groningen, 1929).

[38] A. Strominger and A. Zhiboedov, Gravitational memory, BMS supertranslations and soft theorems, J. High Energy Phys. 01 (2016) 086.

[39] M. Pate, A.-M. Raclariu, and A. Strominger, Gravitational memory in higher dimensions, J. High Energy Phys. 06 (2018) 138.

[40] D. A. Nichols, Spin memory effect for compact binaries in the post-Newtonian approximation, Phys. Rev. D 95, 084048 (2017).

[41] K. Mitman, J. Moxon, M. A. Scheel, S. A. Teukolsky, M. Boyle, N. Deppe, L. E. Kidder, and W. Throwe, Computation of displacement and spin gravitational memory in numerical relativity, Phys. Rev. D 102, 104007 (2020).

[42] B. Maybee, D. O'Connell, and J. Vines, Observables and amplitudes for spinning particles and black holes, J. High Energy Phys. 12 (2019) 156.

[43] E. Herrmann, J. Parra-Martinez, M. S. Ruf, and M. Zeng, Radiative classical gravitational observables at $\mathcal{O}\left(G^{3}\right)$ from scattering amplitudes, J. High Energy Phys. 10 (2021) 148.

[44] A. Manu, D. Ghosh, A. Laddha, and P. V. Athira, Soft radiation from scattering amplitudes revisited, J. High Energy Phys. 05 (2021) 056.

[45] O. Semerák, Spinning test particles in a Kerr field-I, Mon. Not. R. Astron. Soc. 308, 863 (1999). 